Portland State University

PDXScholar

\title{
Employment of Crystallographic Image Processing Techniques to Scanning Probe Microscopy Images of Two-Dimensional Periodic Objects
}

Bill Moon

Portland State University

Follow this and additional works at: https://pdxscholar.library.pdx.edu/open_access_etds Let us know how access to this document benefits you.

Recommended Citation

Moon, Bill, "Employment of Crystallographic Image Processing Techniques to Scanning Probe Microscopy Images of Two-Dimensional Periodic Objects" (2011). Dissertations and Theses. Paper 699.

https://doi.org/10.15760/etd.699

This Thesis is brought to you for free and open access. It has been accepted for inclusion in Dissertations and Theses by an authorized administrator of PDXScholar. Please contact us if we can make this document more accessible: pdxscholar@pdx.edu. 


\title{
Employment of Crystallographic Image Processing Techniques to
}

Scanning Probe Microscopy Images of Two-Dimensional Periodic Objects

\author{
by \\ Bill Moon
}

a thesis submitted in partial fulfillment of the requirements for the degree of

\author{
Master of Science \\ in \\ Physics
}

Thesis Committee:

Peter Moeck, Chair

Mingdi Yan

Raj Solanki

Portland State University

2011 


\begin{abstract}
Thin film arrays of molecules or supramolecules are active subjects of investigation because of their potential value in electronics, chemical sensing, catalysis, and other areas. Scanning probe microscopes (SPMs), including scanning tunneling microscopes (STMs) and atomic force microscopes (AFMs) are commonly used for the characterization and metrology of thin film arrays. As opposed to transmission electron microscopy (TEM), SPMs have the advantage that they can often make observations of thin films in air or liquid, while TEM requires highly specialized techniques if the sample is to be in anything but vacuum. SPM is a surface imaging technique, while TEM typically images a 2D projection of a thin 3D sample. Additionally, variants of SPM can make observations of more than just topography; for instance, magnetic force microscopy measures nanoscale magnetic properties.
\end{abstract}

Thin film arrays are typically two-dimensionally periodic. A perfect, infinite twodimensionally periodic array is mathematically constrained to belong to one of only 17 possible 2D plane symmetry groups. Any real image is both finite and imperfect. Crystallographic Image Processing (CIP) is an algorithm that Fourier transforms a real image into a 2D array of complex numbers, the Fourier coefficients of the image intensity, and then uses the relationship between those coefficients to first ascertain the 2D plane symmetry group that the imperfect, finite image is most likely to possess, and then adjust those coefficients that are symmetry-related so as to perfect the symmetry. A 
Fourier synthesis of the symmetrized coefficients leads to a perfectly symmetric image in direct space (when accumulated rounding and calculation errors are ignored). The technique is, thus, an averaging technique over the direct space experimental data that were selected from the thin film array. The image must have periodicity in two dimensions in order for this technique to be applicable.

CIP has been developed over the past 40 years by the electron crystallography community, which works with 2D projections from 3D samples. Any periodic sample, whether it is $2 \mathrm{D}$ or $3 \mathrm{D}$ has an "ideal structure" which is the structure absent any crystal defects. The ideal structure can be considered one average unit cell, propagated by translation into the whole sample. The "real structure" is an actual sample containing vacancies, dislocations, and other defects. Typically the goal of electron and other types of microscopy is examination of the real structure, as the ideal structure of a crystal is already known from X-ray crystallography. High resolution transmission electron microscope image based electron crystallography, on the other hand, reveals the ideal crystal structure by crystallographic averaging.

The ideal structure of a 2D thin film cannot be easily in a spatially selective fashion examined by grazing incidence X-ray or low energy electron diffraction based crystallography. SPMs straightforwardly observe thin films in direct space, but SPM accuracy is hampered by blunt or multiple tips and other unavoidable instrument errors. Especially since the film is often of a supramolecular system whose molecules are 
weakly bonded (via pi bonds, hydrogen bonds, etc.) both to the substrate and to each other, it is relatively easy for a molecule from the film to adhere to the scanning tip during the scan and become part of the tip during subsequent observation.

If the thin film array has two-dimensional periodicity, CIP is a unique and effective tool both for image enhancement (determination of ideal structure) and for the quantification of overall instrument error. In addition, if a sample of known 2D periodicity is scanned, CIP can return information about the contribution of the instrument itself to the image.

In this thesis we show how the technique is applied to images of two dimensionally periodic samples taken by SPMs. To the best of our knowledge, this has never been done before. Since 2D periodic thin film arrays have an ideal structure that is mathematically constrained to belong to one of the 17 plane symmetry groups, we can use CIP to determine that group and use it for a particularly effective averaging algorithm. We demonstrate that the use of this averaging algorithm removes noise and random error from images more effectively than translational averaging, also known as "lattice averaging" or "Fourier filtering". We also demonstrate the ability to correct systematic errors caused by hysteresis in the scanning process. These results have the effect of obtaining the ideal structure of the sample, averaging out the defects crystallographically, by providing an average unit cell which, when translated, represents the ideal structure. 
In addition, if one has recorded a scanning probe image of a $2 \mathrm{D}$ periodic sample of known symmetry, we demonstrate that it is possible to use the Fourier coefficients of the image transform to solve the inverse problem and calculate the point spread function (PSF) of the instrument. Any real scanning probe instrument departs from the ideal PSF of a Dirac delta function, and CIP allows us to quantify this departure as far as point symmetries are concerned. The result is a deconvolution of the "effective tip", which includes any blunt or multiple tip effects, as well as the effects caused by adhesion of a sample molecule to the scanning tip, or scanning irregularities unrelated to the physical tip.

We also demonstrate that the PSF, once known, can be used on a second image taken by the same instrument under approximately the same experimental conditions to remove errors introduced during that second imaging process.

The preponderance of two-dimensionally periodic samples as subjects of SPM observation makes the application of CIP to SPM images a valuable technique to extract a maximum amount of information from these images. The improved resolution of current SPMs creates images with more higher-order Fourier coefficients than earlier, "softer" images; these higher-order coefficients are especially amenable to CIP, which can then effectively magnify the resolution improvement created by better hardware. 
The improved resolution combined with the current interest in supramolecular structures (which although 3D usually start building on a 2D periodic surface) appears to provide an opportunity for CIP to significantly contribute to SPM image processing. 


\section{Acknowledgments}

This thesis would have been quite impossible without the assistance of many people. I will thank some of them here, but there are many others at PSU and elsewhere too numerous to name.

First, my advisor, Peter Moeck, for his guidance, ideas, encyclopedic knowledge of crystallography, and encouragement to embark upon this project and willingness to see it through.

The Nano-Crystallography Group, Sergei Rouvimov, Jack Straton, have all been a creative source as well as role models for the pursuit of science.

The members of my thesis committee, Mingdi Yan and Raj Solanki, who are generously spending their time on assessing this work and offering suggestions for improvement.

During my time as a graduate student at Portland State I was given the opportunity to spend a summer working at the University of California, Davis, for Prof. Nigel Browning and another summer at the Technical University of Chemnitz for Prof. Dr. Sc. Michael Hietschold. I want to thank Prof. Browning and Prof. Dr. Hietschold for welcoming me into their groups. Dr. Steffen Schulze and Prof. Dr. Hietschold at Chemnitz were most generous in allowing me access to their instruments.

I want to thank Andres La Rosa and Eric Sánchez at Portland State for access to their instruments and the invaluable advice they have given me. 
Thank you to the other professors at PSU who have advised me and encouraged me, and to my fellow graduate students for their fellowship and assistance.

Finally, to my wife Lorraine for her patience, encouragement, and faith in this endeavor. 


\section{Table of Contents}

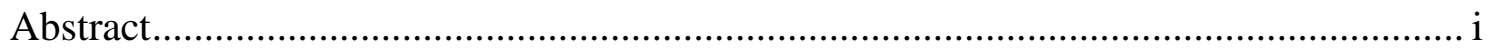

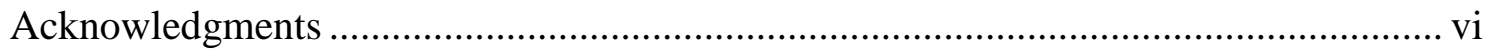

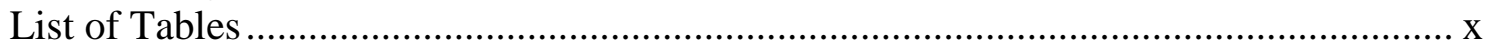

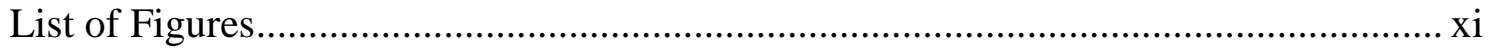

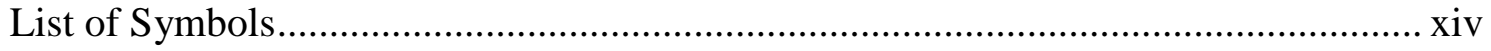

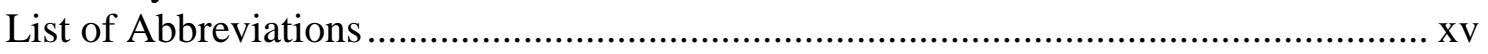

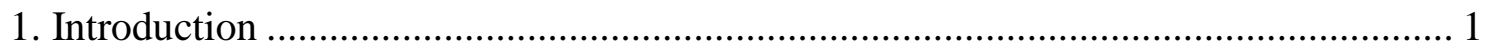

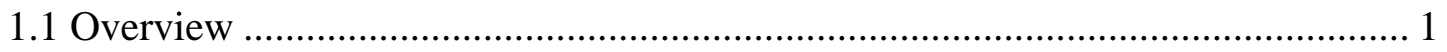

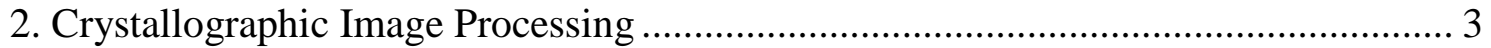

2.1 The Fourier Transform .................................................................................. 3

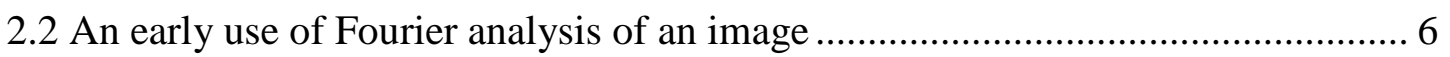

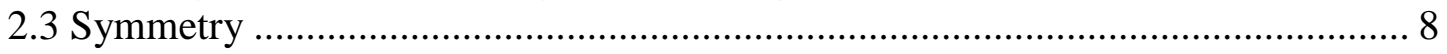

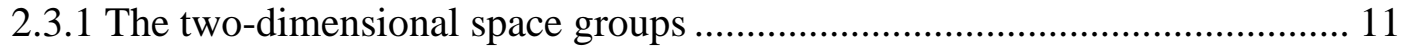

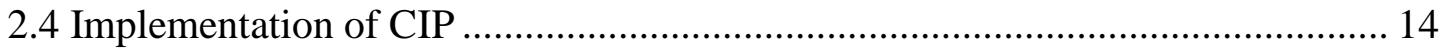

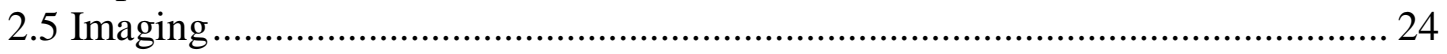

2.6 Application of CIP to High-Resolution Transmission Electron Microscope

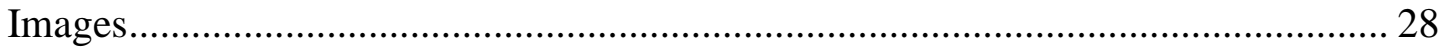

2.7 CIP applications to Scanning Probe Microscope images .................................. 33

2.7.1 Scanning Tunneling Microscopy............................................................. 35

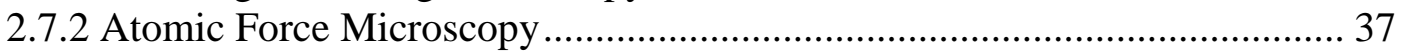

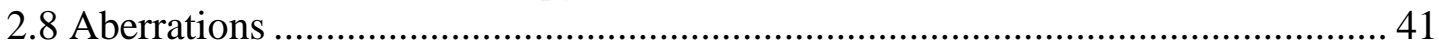

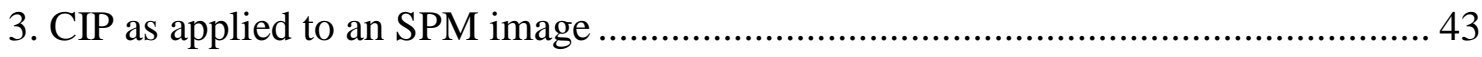

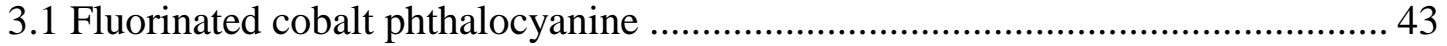

3.1.1 Possible orientation of molecule on M16 ................................................... 44

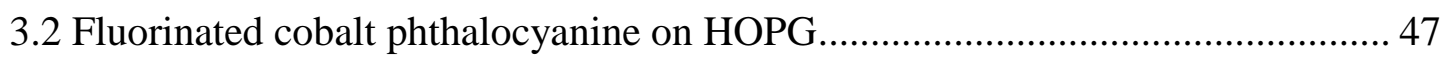

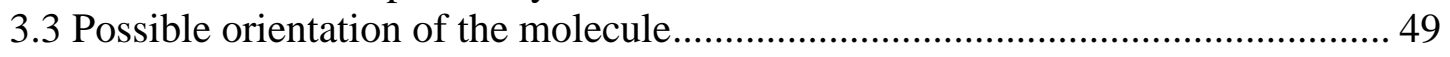

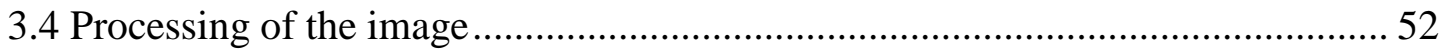

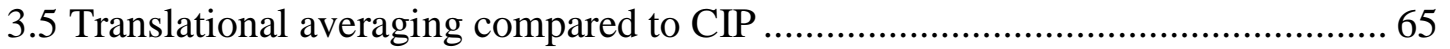

3.5.1 Effectiveness of CIP compared to translational averaging.......................... 68

3.6 CIP restoration of an image with systematic error ........................................ 74

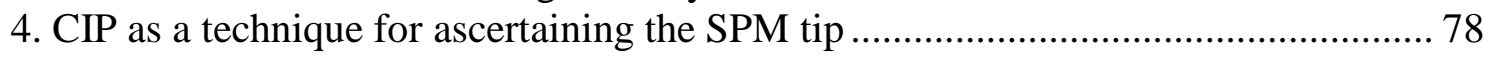

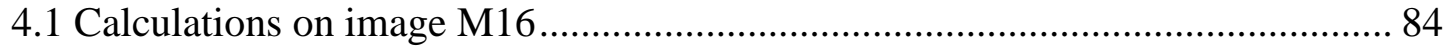

4.2 Calculations on image M17 using the PSF from M16 ................................... 91

4.2.1 Zero values in the PSF....................................................................... 91

4.2.2 Production of corrected M17 coefficients ................................................. 93

4.2.3 Production of corrected M17 coefficients using a bias .............................. 97

4.3 Real space reconstruction of the effective tip PSF ......................................... 100

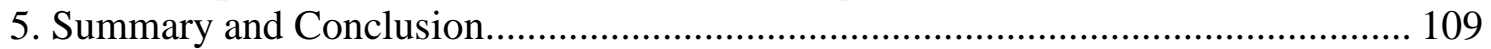

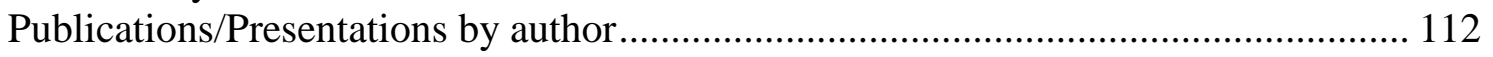

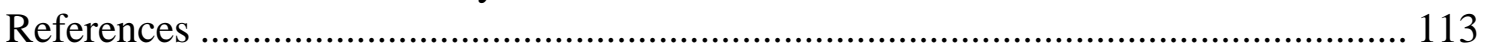


Appendix A - Source code for simulated images ............................................. 115

Appendix B -- Complex number notation..... 


\section{List of Tables}

$\begin{array}{lll}\text { table } & \text { description } & \text { page } \\ 2.1 & \text { the 17 two-dimensional plane groups } & 13 \\ 3.1 & \text { residuals of three separate areas of figure 3.1 } & 52 \\ 4.1 & \text { 24 largest Fourier coefficients of M16 } & 87 \\ 4.2 & \text { 53 Fourier coefficients of the M16 point spread function } & 90 \\ & \text { (PSF) } & 94 \\ 4.3 & \text { M17 Fourier coefficients with M16 corrections } & 97 \\ 4.4 & \text { M17 Fourier coefficients with biased M16 corrections } & 99 \\ 4.5 & \text { residuals for two different M17 correction schemes } & 102 \\ 4.6 & \text { 10 highest M16 PSF coefficients ranked by } P_{\text {tip }} & 104 \\ 4.7 & \text { 10 highest M16 PSF coefficients after weighting by } I_{\text {raw }} & 106 \\ 4.8 & \text { PSF calculation for figure 3.35 (open loop) } & 107 \\ 4.9 & \text { PSF calculation for figure 3.34 (closed loop) }\end{array}$




\section{List of Figures}

figure description

page

2.1 Arnold Klug's virus image, with Fourier transforms 7

2.2 3D unit cell showing axes and angles, labeled 9

2.3 the 17 two-dimensional plane groups 12

$\begin{array}{lll}2.4 & \mathrm{MgO} \text { diffraction pattern } & 17\end{array}$

2.5 STM image of $\mathrm{F}_{16} \mathrm{CoPc}$ showing amplitude, phase 17

$\begin{array}{lll}2.6 & \text { the five possible two-dimensional lattices } & 19\end{array}$

2.7 HRTEM images of boron structures 30

$2.8 \quad c 2 \mathrm{~mm}$ enforced images of a boron structure 31

2.9 CIP enforced images of boron structures with molecule 31

2.10 Simulated boron structure images 32

$\begin{array}{lll}2.11 & \text { diagram of STM }\end{array}$

2.12 tunneling potential barrier 36

2.13 AFM tip and cantilever diagram 37

2.14 tunneling current and interatomic forces at short distances 40

$\begin{array}{lll}3.1 & \text { Phthalocyanine structural formula } & 43\end{array}$

3.2 Cobalt phthalocyanine structural formula 43

$\begin{array}{lll}3.3 & \text { Fluorinated cobalt phthalocyanine sketch }\end{array}$

3.4 M16 reciprocal axes 45

3.5 M16 centered reciprocal axes 46

$3.6 \quad$ M16 residuals $\mathrm{cm} \quad 46$

3.7 STM image of fluorinated cobalt phthalocyanine on HOPG 47

$\begin{array}{lll}3.8 & \text { STM image of pure HOPG }\end{array}$

3.9 CIP analysis of pure HOPG 48

3.10 diagram of possible orientation of $\mathrm{F}_{16} \mathrm{CoPc}$ in layer 50 
3.11 Amplitude portion of Fourier transform of three separate areas 51 of figure 3.7

3.12 Fourier transform showing reciprocal axes 53

3.13 possible plane groups and residuals for figure 3.7, and $p 1 \quad 58$ enforced reconstruction

$3.14 \quad p 2$ enforced reconstruction of figure $3.7 \quad 61$

$\begin{array}{lll}3.15 & p 4 \text { enforced reconstruction of figure } 3.7 & 63\end{array}$

$3.16 \quad p 4 m m$ enforced reconstruction of figure 3.7, including detail 63

3.17 simulated $p 4 m m$ image, $4 \times 4$

3.18 simulated $p 4 m m$ image, $4 \times 4$ with noise 65

$3.19 \quad p l$ enforcement noisy image 66

$3.20 \quad$ closeup of $p l$ enforcement 66

$\begin{array}{lll}3.21 & \text { p4mm enforcement noisy image } & 67\end{array}$

$3.22 \quad$ closeup of $p 4 m m$ enforcement 67

$\begin{array}{lll}3.23 & \text { simulated } p 4 m m \text { image, } 6 \times 6 & 68\end{array}$

3.24 simulated $p 4 m m$ image, $6 \times 6$ with noise 68

$\begin{array}{lll}3.25 & p l \text { enforcement noisy image, } 6 \times 6 & 70\end{array}$

3.26 closeup of $p l$ enforcement, $6 \times 6 \quad 70$

$3.27 \quad p 4 m m$ enforcement noisy image, $6 \times 6 \quad 70$

3.28 closeup of $p 4 m m$ enforcement, $6 \times 6 \quad 70$

3.29 simulated $p 4 m m$ image, $50 \times 50 \quad 71$

$3.30 \quad$ simulated $\mathrm{p} 4 \mathrm{~mm}$ image, $50 \times 50$ with noise 71

$3.31 \quad$ closeup of $p 1$ and $p 4 m m$ enforcement, $50 \times 50$

3.32 simulated $p 4 m m$ image, 50×50 with systematic error 73

3.33 closeup of $p 1$ and $p 4 m m$ enforcement, $50 \times 50$ systematic 73

$\begin{array}{lll}3.34 & \text { AFM image 256×256 pixel closed-loop } & 75\end{array}$

3.35 AFM image 256×256 pixel open-loop 75

$\begin{array}{lll}3.36 & 76\end{array}$ 
3.37 closed-loop FT, residuals, p4mm enforcement 76

$\begin{array}{lll}3.38 & \text { open-loop FT detail }\end{array}$

3.39 open-loop FT, residuals, $p 4 m m$ enforcement 77

$\begin{array}{lll}4.1 & \text { classic double tip image } & 80\end{array}$

4.2 STM images M16 and M17, F 16 CoPc on HOPG 80

4.3 M16 and its Fourier transform 84

4.4 CRISP output, M16, showing reciprocal axes 85

4.5 possible plane groups and residuals for M16 86

4.6 p3m1 enforced reconstruction of M16 using 24 strongest 90

4.7 p3ml enforced reconstruction using 53 coefficients 90

4.8 M17 and its Fourier transform 93

4.9 M17 corrected by the M16 PSF 95

4.10 CRISP output corrected M17 showing residuals 96

4.11 three corrections of M17 with various values for $(3,7) \quad 96$

4.12 M17 corrected by a biased M16 PSF 98

4.13 the (3,7) spot on the Fourier transform of M17 99

$\begin{array}{lll}4.14 & 101\end{array}$

$\begin{array}{lll}4.15 & \text { plot of M16 PSF } & 101\end{array}$

4.16 plot of M16 weighted PSF, two units cells 103

4.17 closeup contour plot M16 PSF, unweighted and weighted 104

$\begin{array}{lll}4.16 & \text { plot of figure 3.35 (open loop )PSF }\end{array}$

$\begin{array}{lll}4.17 & \text { plot of figure } 3.34 \text { (closed loop) PSF }\end{array}$ 


\section{List of Symbols}

\begin{tabular}{|c|c|}
\hline$F(H, K)$ & a complex function of two integers in Fourier space \\
\hline$f(x, y)$ & a real function of two real numbers in real space \\
\hline$p$ & in a Hermann-Maugain group symbol, a primitive lattice in $2 \mathrm{D}$ \\
\hline$c$ & in a Hermann-Maugain group symbol, a centered lattice in 2D \\
\hline$A_{\text {res }}$ & $\begin{array}{l}\text { sum of amplitude residuals of Fourier coefficients for a particular plane } \\
\text { group }\end{array}$ \\
\hline$\varphi_{\text {res }}$ & $\begin{array}{l}\text { sum of phase residuals of Fourier coefficients for a particular plane } \\
\text { group }\end{array}$ \\
\hline$R A \%$ & $\begin{array}{l}\text { comparison of symmetry-related Fourier coefficients which, for a } \\
\text { particular plane group, should be equal }\end{array}$ \\
\hline Ao/Ae & $\begin{array}{l}\text { for plane groups with coefficients "forbidden" due to symmetry, ratio of } \\
\text { amplitude of (observed forbidden coefficients)/( allowed coefficients) }\end{array}$ \\
\hline$i, o, p$ & $\begin{array}{l}\text { real-space real-valued two-dimensional functions representing image, } \\
\text { object, and tip ( } p \text { is also known as the effective point spread function) }\end{array}$ \\
\hline$I, O, P$ & $\begin{array}{l}\text { Fourier space complex-valued two dimensional functions representing } \\
\text { Fourier transforms of image, object, and tip }\end{array}$ \\
\hline
\end{tabular}




\section{List of Abbreviations}

$\begin{array}{ll}\text { AFM } & \text { Atomic force microscope } \\ \text { AIC } & \text { Akaike information criterion } \\ \text { CIP } & \text { Crystallographic image processing } \\ \text { CTF } & \text { Contrast transfer function } \\ \text { DFT } & \text { Discrete Fourier transform } \\ \text { FFT } & \text { Fast Fourier transform } \\ \text { HOPG } & \text { Highly-oriented pyrolytic graphite } \\ \text { HRTEM } & \text { High resolution transmission electron microscopy } \\ \text { LDOS } & \text { Local density of states } \\ \text { PSF } & \text { Point spread function } \\ \text { SEM } & \text { Scanning electron microscopy } \\ \text { SPM } & \text { Scanning probe microscope } \\ \text { STM } & \text { Scanning tunneling microscope } \\ \text { TEM } & \text { Transmission electron microscopy } \\ \text { UHV } & \text { Ultra-high vacuum }\end{array}$




\section{Introduction}

\subsection{Overview}

This thesis is intended to first describe an existing method of image processing, "crystallographic image processing" (CIP), which historically has been used to extract information from high resolution transmission electron microscopy (HRTEM) images of periodic objects, and then to show that this same technique can extract useful information from certain scanning probe microscopy (SPM) images.

The application of this technique to SPM images has two significant benefits. First, if the SPM image is of a sample that is two-dimensionally periodic, this thesis will show that CIP can help extract signal from noise. This is done by averaging the unit cells together, which suppresses noise and random error in the image. Translational averaging of periodic image elements is a well-known technique; however CIP adds additional processing steps. In addition to translational averaging of unit cells, by determining the plane group symmetry of the sample and enforcing that symmetry upon the uncorrected image one can enhance detail over and above translational averaging. Certain systematic errors such as image bow or a trapezoidal distortion can also be eliminated by CIP. Averaging techniques suppress the image of any actual defects in the sample, which may or may not be desirable depending upon what the user is investigating. The CIP technique is well-known for electron microscopy; this thesis is intended to show its value for SPM as well. 
Second, as will be discussed below, an SPM instrument has a tip that is ideally a point but often is multiple points or an irregular shape - and knowledge of the configuration of this tip is essential in interpreting an SPM image. This information is most useful if it can be obtained while the tip is in situ, (installed in a working instrument). There are existing techniques such as blind reconstruction [1] for ascertaining this shape, as well as direct examination by SEM (scanning electron microscopy). This thesis will show that CIP can be used to make this shape determination by taking advantage of the symmetry of a highly periodic calibration sample, and is a unique and valuable approach for making this determination. 


\section{Crystallographic Image Processing}

\subsection{The Fourier Transform}

The Fourier transform is an integral transform that operates on a complex function of $n$ variables to produce another complex function of $n$ variables. Since images can be considered two-dimensional density functions, $n=2$ for image processing, and given the monotonic nature of a monochrome density function (this thesis does not discuss color or false-color images) the input function is real-valued rather than complex. The output of the transform is complex although certain inputs will force all of the imaginary components of the output to zero.

There are equivalent formulations of the Fourier transform. This thesis will use the "crystallographic convention" in which the direct transform is:

$$
F(H, K)=\int f(x, y) \exp (2 \pi i(x H+y K)) d x d y
$$

that takes the complex function $f$ in what is commonly referred to as "real space" to the complex function $F$ in what is called "reciprocal space" or "Fourier space", and the inverse transform is:

$f(x, y)=\int F(H, K) \exp (-2 \pi i(H x+K y)) d H d K$

Note the negative sign in the exponential is the only computational difference. The two functions $f(x, y)$ and $F(H, K)$ are known as "Fourier transform pairs" [2]. The 
variables $x$ and $y$ can be viewed as components of a two-dimensional vector in real space, while $H$ and $K$ are components of a vector in reciprocal space. As used in CIP, an $H K$ vector represents a particular crystallographic direction in the sample.

There are other conventions regarding the exact definition of the Fourier transform. In one common convention sometimes used by physicists the exponential portion of the forward Fourier transform is $e^{-2 \pi \mathrm{i}}$ while the inverse transform has an exponential of $e^{2 \pi \mathrm{i}}$. Fourier pairs retain their relationship regardless of which convention is used, but the same convention must be consistently applied to one problem

The Fourier transform operates on continuous functions and generates a (usually) continuous result. Image processing is done on a discrete two-dimensional array of real numbers that correspond to the intensity of the associated pixel (a value can also be assigned to color, which is disregarded here). The Discrete Fourier Transform (DFT) is the applicable technique for discrete functions. In one dimension, compare the Fourier Transform:

$$
F(H)=\int f(x) \exp (2 \pi i(x H)) d x
$$

to the Discrete Fourier Transform:

$$
F(H)=\sum_{n=0}^{N-1} f\left(x_{n}\right) \exp \left(2 \pi i\left(\frac{n H}{N}\right)\right)
$$

where $N$ is the number of discrete data points $x_{n}$ input. The Fast Fourier Transform (FFT) is the usual implementation of the DFT algorithm, as it is computationally 
efficient, although recent improvements in computer speed have to some degree alleviated the need for the most efficient algorithm possible.

The Fourier transform as applied to crystallographic image processing maps real space to a spatial frequency space (usually referred to as "reciprocal space" in image processing, or "Fourier space"). The data (output from the DFT) in this reciprocal space is a $2 \mathrm{D}$ array of complex numbers, indexed by the $H$ and $K$ components. This data is most easily visualized (and plotted) in polar form. The complex number is in the form $r e^{i \theta}$ instead of $x+i y$. The $r$ coordinate in this case is the amplitude of the transform at this $(H, K)$ point and the $\theta$ is the phase. If the sample is periodic, which is the usual case when CIP is used, the amplitude of the reciprocal space array contains spots (mathematical points in the ideal case) that correspond to spatial periodicities in the real space data.

An image containing two-dimensional periodicities in the $x$ and $y$ directions of real space will thus have a regular two-dimensional spot pattern in the amplitude coordinate of Fourier space. The symmetry information present in an object is also present in the Fourier transform.

A plot of a Fourier transform of a real-space image usually shows only information about the amplitude portion of the transform. What one sees is the intensity and location of the spots. The intensity corresponds to the square of the amplitude of the Fourier coefficient. The phase portion of the coefficient, which provides information about the two-dimensional translational symmetry of the lattice, is fully half of the 
information output of a Fourier transform, and is certainly used in CIP (it is more critical than amplitude) but is typically not plotted as an image because it conveys little information to the human eye. See the right portion of figure 2.5 for an example of a phase plot.

\subsection{An early use of Fourier analysis of an image}

Aaron Klug received the 1982 Nobel Prize in Chemistry "for his development of crystallographic electron microscopy and his structural elucidation of biologically important nucleic acid-protein complexes" [3].

Klug was investigating the structure of viruses and had turned to electron microscopy with the intent of using direct images. Many of the images were difficult to interpret because the depth of field of the instrument (as is typical in electron microscopy) permitted the entire vertical depth of the sample to appear in focus, essentially creating a two-dimensional projection of the three dimensional object. The resulting image was a superposition of the image of the front wall, back wall, and internal structure of the object (Figure 2.1).

His solution involved taking the Fourier transform of the image. 

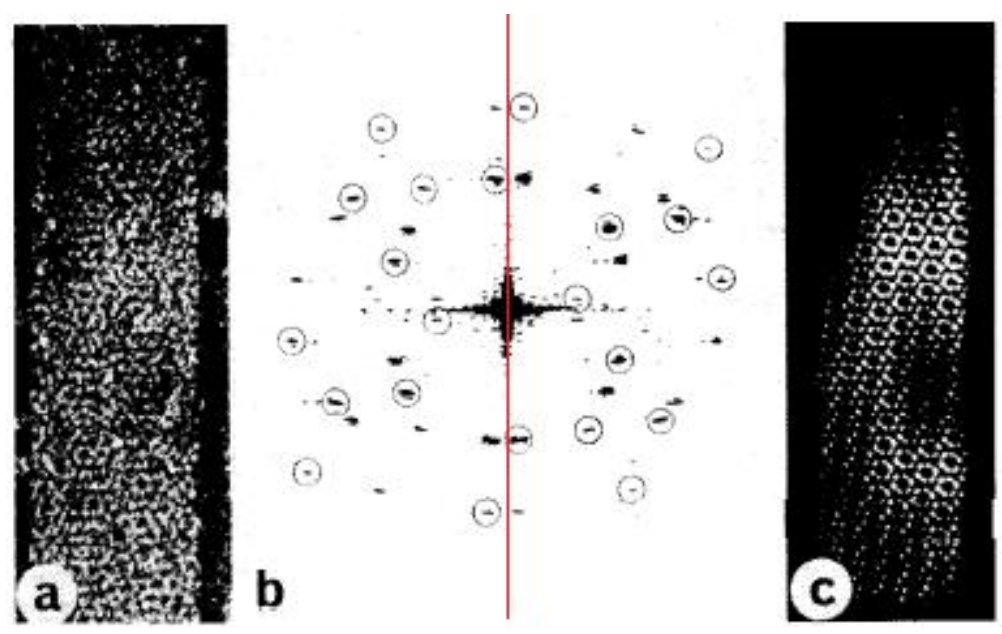

Figure 2.1

(a) Original electron microscope image;

(b) amplitude portion of diffraction pattern of image with selected spots circled; note there is a vertical mirror line through the center (shown in red), and the uncircled spots form a close, but not exact, mirror reflection of the circled spots;

(c) inverse Fourier reconstruction using just circled spots.

figure from [3]

Because one of Klug's samples was largely periodic with two-dimensional symmetries, (a spiral structure extracted from a phage virus), the Fourier transform consisted of symmetric patterns of spots mixed with noise. Most significantly, since Klug's image was the projection of the front wall and back wall of the virus on top of one another, both of which were two-dimensional periodic in nature, with little or no internal structure between, the Fourier transform was visibly made up of two mirrorimage symmetric patterns of spots (see Figure 2.1). Klug's solution was to select one set of spots, eliminate the other set (along with the noise), and perform a reverse Fourier transform on the result. This procedure produced a clear image of one of the walls of the structure. 
Any two dimensional image can be Fourier transformed, and useful information can be extracted even if the image is nonperiodic. CIP depends upon periodicity to be useful and cannot address nonperiodic samples. Even though Klug's sample was periodic, his technique did not constitute CIP. But it is an illustration of the power of the Fourier transform in extracting and presenting information about the periodicity of an image.

\subsection{Symmetry}

A crystal, by definition, is a three-dimensional array of identical atomic units called "unit cells" extending in all directions. The unit cells can be made up of one atom or thousands, but they are arranged regularly so that, disregarding surfaces and defects, an observation made from a particular location in any unit cell is identical to the same observation from the same location in any other unit cell. This is in contrast to an amorphous solid, a glass, in which there is no long-range order of the atoms. The definition of the unit cell of a particular crystal is arbitrary, there are an infinite number of possible unit cells, but generally the simplest cell that exhibits the maximum symmetry is agreed upon. Geometry limits the possible arrangements of unit cells in a crystal to a finite number of "lattices".

The lattice is a mathematical construct. To quantify locations in the lattice, a linear basis is constructed for the crystal by placing the origin at one corner of a unit cell and choosing three different edges that share the origin as axes, that are generally labeled as $a, b$ and $c$. See Figure 2.2. These axes need not be orthogonal nor does the 
unit length have to be the same for each axis. The angles between the axes are labeled $\alpha$, $\beta$ and $\gamma$.

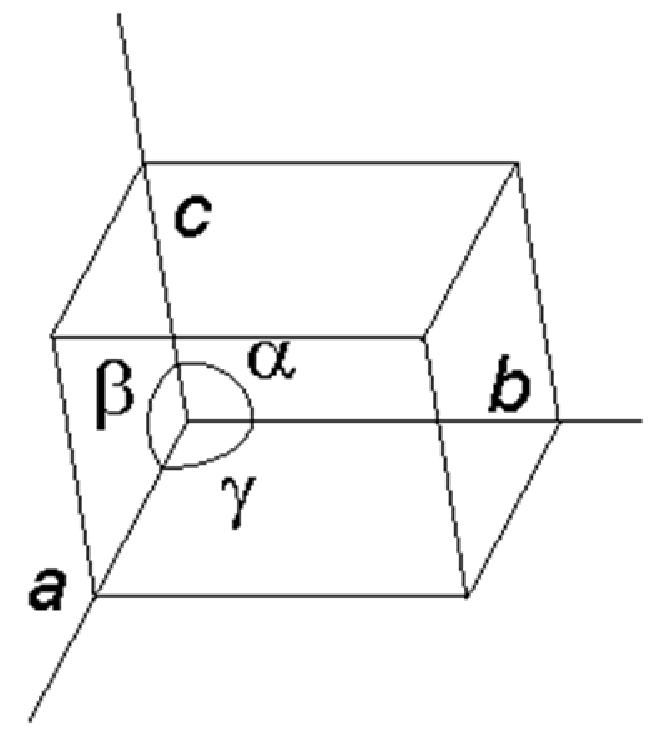

Figure 2.2. A three-dimensional unit cell. Standard right-hand axes; angles can be orthogonal and unit lengths equal, need not be. (from http://xrayweb.chem.ou.edu, University of Oklahoma)

This provides a convenient way to describe the location of atoms within the unit cell; analogous to Cartesian coordinates; one normalizes the edges, so $a, b$, and $c$ are the unit vectors for the crystal. The length of each is 1 but note that in Cartesian space it is not necessary that $a=b=c$. Then the location of each atom is its fractional distance along the edge of each axis.

In addition to the direct lattice already described, it is helpful to describe a "reciprocal lattice". This is a three-dimensional mathematical construct analogous to the direct lattice but it exists in Fourier space. The three linear basis vectors are called $a^{*}$, $b^{*}$, and $c^{*}$ and each one is perpendicular to the face of the unit cell normal to the corresponding real space unit vector. Note that the reciprocal space vectors are only parallel to real space vectors when the crystal symmetry is cubic, tetragonal or 
orthorhombic. The units of reciprocal space are of inverse length and by definition the spacing between lattice points is the inverse of the corresponding space between real space points.

Ideal crystals possess symmetry - that is, after certain translations, rotations, reflections, inversions, or rotoinversions are performed, the crystal is in a new orientation, and it is indistinguishable from the old orientation. All real crystals have defects that prevent strict symmetry, but are reasonably close to symmetric.

Symmetry is mathematically described by the specific symmetry operations that leave the crystal unchanged. When the symmetries of a crystal have been ascertained it can be assigned to a space group. For three dimensional objects, when all possible symmetries (including translational) are accounted for, there are only 230 possible space groups. This is a mathematical limitation imposed by the geometry of three dimensional space.

Although objects in real space exist in three dimensions, their projections exist in two dimensions, and symmetry exists in 2D as well and can be similarly quantified. There are also physical entities such as thin films and surfaces of materials that have features in three dimensions but present a two-dimensional aspect to the observer, and their symmetry can be described this way. The vital reason why CIP can produce useful results is that there are only 17 possible space groups in two dimensions, analogous to the 230 space group limitation in three dimensions. (One of these $2 \mathrm{D}$ groups, the $p 1$ group, describes an object that has only translational symmetry; it has no rotation, 
mirror, or glide elements and thus there are 16 space groups in two dimensions that have a higher symmetry.) Therefore if one is curious as to the structure of a twodimensional film or surface and knows that it is periodic: (1) it is a fact that the structure must possess one of these 17 possible symmetries, and (2) to the extent that it does not, it must vary from periodicity, because of either experimental error, a defect in the structure, or a false assumption that the structure is periodic.

The plan of attack of CIP is to (through Fourier analysis) take an imperfect image, ascertain which of the 17 groups most closely matches its symmetry, and correct the image by "enforcing" the correct symmetry.

\subsubsection{The two-dimensional space groups}

There are various nomenclatures to describe space groups. The International Tables for Crystallography [4] use Hermann-Mauguin symbols. The symbol itself communicates information about the centering type of the conventional cell and the symmetry of the group. The initial letter " $p$ " means the lattice is "primitive", meaning

there is only one lattice point per cell. A centered lattice with initial letter " $c$ " has two lattice points per cell in two dimensions and can potentially be described with a simpler primitive lattice, but at the cost of disregarding some symmetry elements. See Figure 2.3 and Table 2.1 . 


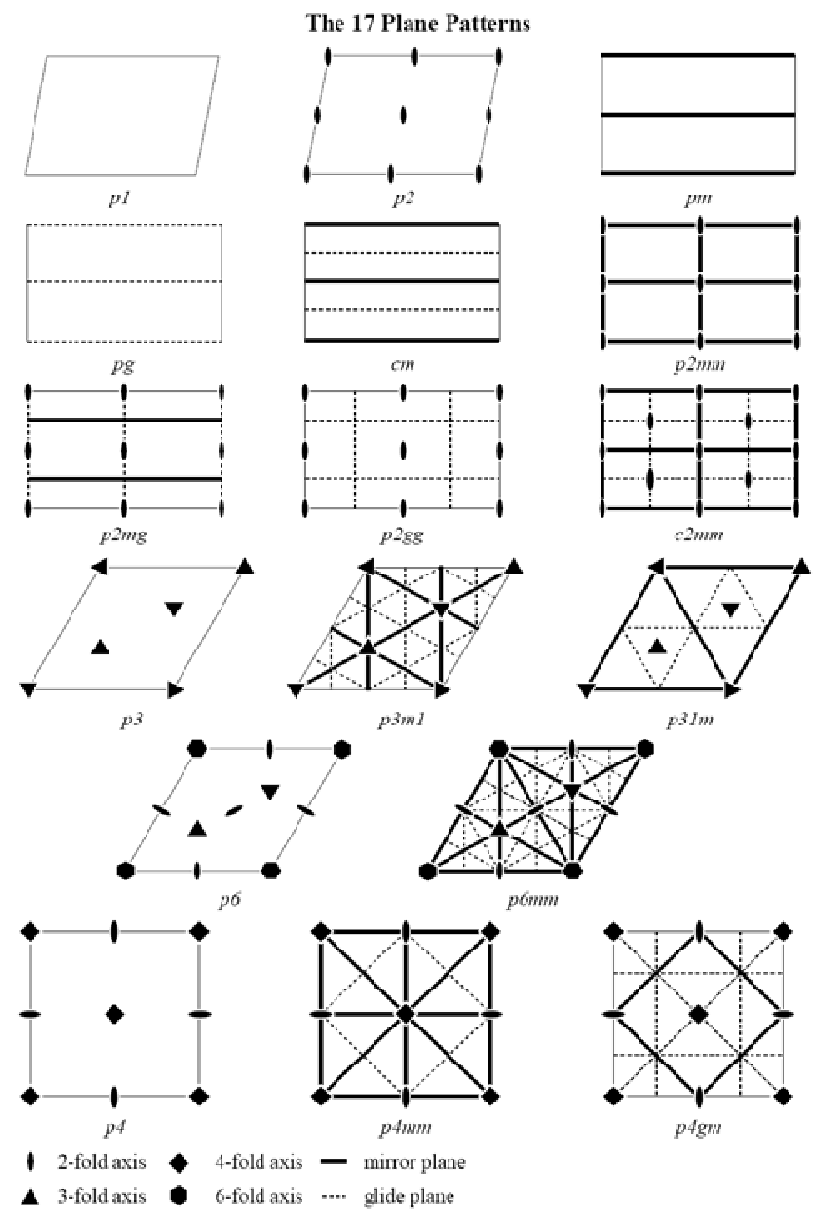

Figure 2.3. Drawings of the 17 two-dimensional space groups with ordering slightly different from Intl Tables for Crystallography.

http://www.science.smith.edu/departments/Geology/Min_jb/Plane_Patterns.pdf, Smith College 
Table 2.1. List of the 17 two-dimensional plane groups.

\begin{tabular}{|c|c|c|}
\hline $\begin{array}{l}\text { group } \\
\text { number }\end{array}$ & short Hermann-Mauguin symbol & comments \\
\hline 1 & $p 1$ & no symmetry other than translation \\
\hline 2 & p2 & four twofold axes \\
\hline 3 & $p m$ & no rotations, two parallel mirror lines \\
\hline 4 & $p g$ & as $p m$ with glides instead of mirrors \\
\hline 5 & $\mathrm{~cm}$ & $\begin{array}{l}\text { can describe with primitive cell but with } \\
\text { less symmetry; two parallel mirrors, two } \\
\text { glides between mirrors }\end{array}$ \\
\hline 6 & $p 2 m m$ & $\begin{array}{l}\text { two perpendicular mirrors, twofold axes } \\
\text { where mirror lines intersect }\end{array}$ \\
\hline 7 & $p 2 m g$ & $\begin{array}{l}\text { two parallel mirrors, two glides } \\
\text { perpendicular to mirrors, twofold axes } \\
\text { on glides }\end{array}$ \\
\hline 8 & $p 2 g g$ & $\begin{array}{l}\text { two glides in each of two perpendicular } \\
\text { directions, twofold axes on glides }\end{array}$ \\
\hline 9 & $c 2 m m$ & $\begin{array}{l}\text { non-primitive cell; two perpendicular } \\
\text { mirrors, a twofold axis not on a mirror, } \\
\text { two that are on mirrors }\end{array}$ \\
\hline 10 & $p 4$ & $\begin{array}{l}\text { two fourfold axes, one twofold axis, no } \\
\text { reflections }\end{array}$ \\
\hline 11 & $p 4 m m$ & as $p 4$ with four mirrors, two glides \\
\hline 12 & p4gm & as $p 4$ with two mirrors, multiple glides \\
\hline 13 & p3 & three threefold axes, no reflections \\
\hline 14 & p3m1 & $\begin{array}{l}\text { three threefold axes, mirrors through } \\
\text { each one, glides parallel to mirrors }\end{array}$ \\
\hline 15 & p31m & $\begin{array}{l}\text { three threefold axes, mirrors but one axis } \\
\text { without a mirror, multiple glides }\end{array}$ \\
\hline 16 & p6 & $\begin{array}{l}\text { one sixfold axis, two threefold, three } \\
\text { twofold, no reflections }\end{array}$ \\
\hline 17 & p6mm & as $p 6$ with six mirrors, six glides \\
\hline
\end{tabular}

Any periodic two-dimensional image must fit into one of these categories.

There is a subset of the unit cell of a space group called the "asymmetric unit"; it is the smallest closed part of the space group that can fill all of space by application of the symmetry operations. The asymmetric unit, combined with the symmetry operations, contains enough information to recreate the entire unit cell and therefore the 
entire crystal. The higher the symmetry of the plane group, the smaller the relative size of the asymmetric unit.

The multiplicity of a group refers to the "general position" within the unit cell, which is an arbitrary locus not on a symmetry element, and is the number of times that the locus will be copied within the unit cell as a result of all of the symmetry operations. Intuitively, it is a measure of the "quantity of symmetry" that a particular space group has. A highly symmetric group is p6mm and its general position has a multiplicity of 12 . The asymmetric unit of $p 6 \mathrm{~mm}$ has an area of $1 / 12$ of the area of the whole unit cell, a reciprocal relationship that holds for any primitive group.

As stated earlier, the plan of CIP is to take a periodic image, treat it as a twodimensional array of pixels, and ascertain its plane group. One method, as used in the CRISP program of Calidris, Inc., uses the Fast Fourier Transform (FFT) implementation of the Discrete Fourier Transformation (DFT) to operate on the image. The resulting transform has peaks or spots corresponding to periodicities in the image. These spots, which exist in reciprocal space, are matched to a possible reciprocal lattice; either automatically or by human intervention.

\subsection{Implementation of CIP}

One possible sequence of operations to actually perform CIP is as follows.

1. Create a reciprocal lattice. Take an image or portion of image as a $2^{\mathrm{n}} \times 2^{\mathrm{n}}$ array of pixels, each with a value representing the gray-scale intensity of the image at that point. This is an array of integers. In order to calculate the two dimensional Fourier 
transform of this array, use one of the Fast Fourier Transform implementations of the Discrete Fourier Transform. Following is the form of the DFT:

$$
F(H, K)=\sum_{x=0}^{N-1} \sum_{y=0}^{M-1} f(x, y) \exp \left(2 \pi i\left(\frac{x H}{N}+\frac{y K}{M}\right)\right)
$$

The FFT is known as a computationally efficient algorithm for calculating this transformation. The resulting transform is a $2^{\mathrm{n}} \times 2^{\mathrm{n}}$ array of complex numbers. A complex number can be expressed either in the form $a+b i$ where $a$ is the real part and $b$ is the imaginary part, or in the form $r e^{\mathrm{i} \varphi}$ where $r$ is the magnitude of the complex number on an Argand diagram and $\varphi$ is the phase angle. These are related by:

$$
\begin{aligned}
& r=\sqrt{a^{2}+b^{2}} \\
& \varphi=\arctan \frac{b}{a}
\end{aligned}
$$

(where the principal value of the arctangent function ranges from $-\pi / 2$ to $\pi / 2$ )

For the purposes of CIP the $r e^{i \varphi}$ format is most useful and each ordered pair of the array contains the amplitude of the Fourier component and the phase. This can be viewed as a two-dimensional array of complex numbers or two arrays of real numbers.

Plot an image of the FFT (in order for the user to see and interact with) as a pixel at each $x^{*}-y^{*}$ location with the pixel intensity equal to the square of the amplitude part of the transform at that point. Assuming the original image had two-dimensional periodicity, the FFT image will consist of bright spots (mixed with noise and transforms of nonperiodic elements). The left portion of Figure 2.4 is an example. 
2. Characterize the reciprocal lattice. The spots form a lattice in reciprocal space. There are two tasks to perform with these spots. The first is to accurately determine their location. This is done with existing software that finds the peaks in the amplitude components of the two dimensional array, although the location can be fine-tuned with subpixel interpolation and by fitting the peaks to a grid by a least-squares method [5].

Determining the exact location of the amplitude peaks is particularly important when one considers that this location is also used to identify the phase value associated with the spot, and the values of the phases do not peak but can vary significantly from pixel to pixel. (One can plot an image of the phases similar to the usual image of the amplitudes, but the "spots" are not conspicuous or in some cases nonexistent.) Thus a one pixel error in the location of the amplitude peak, which may not greatly impact the amplitude value itself, can result in a significant error in the phase.

Figure 2.5 shows an STM image on the left (this image will be seen and analyzed later in the thesis), the Fourier amplitude squared in the middle, and the Fourier phase on the right. The spots are clearly visible in the amplitude plot but invisible to the human eye in the phase plot. 

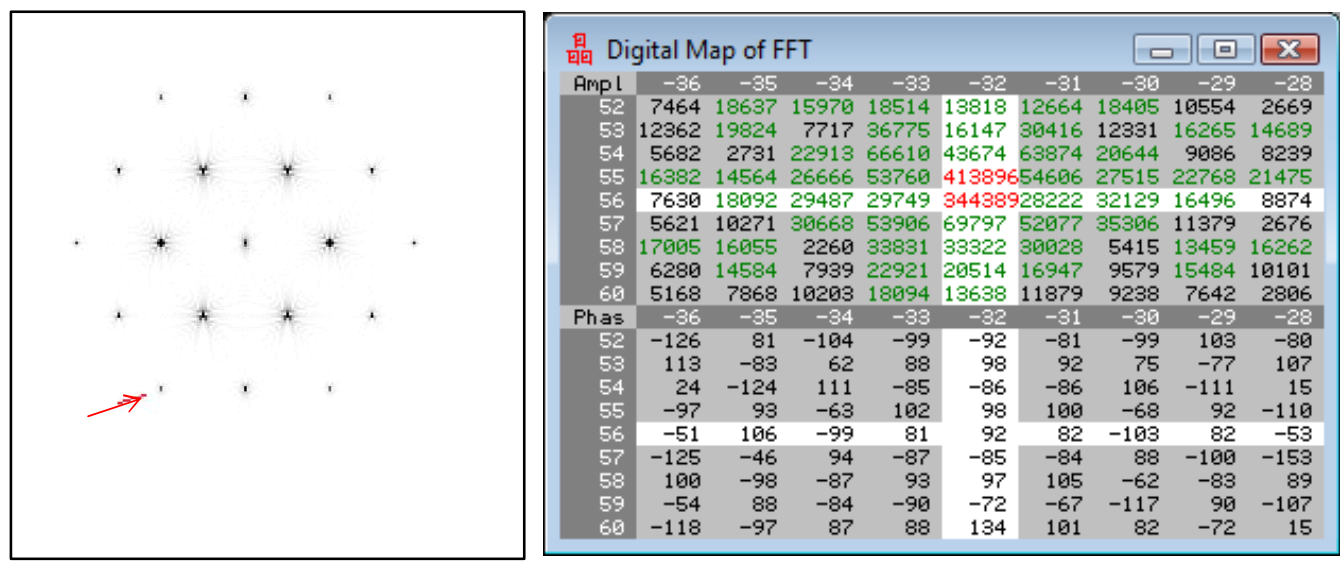

Figure 2.4. Two presentations of a Fourier transformation of a simulated HRTEM image of $\mathrm{MgO}(111)$ (left) image of the amplitude portion of the Fourier transformation (black/white inverted for readability). One of the spots in the lower left has a small red arrow pointing it out.(right) the vicinity of the marked spot, showing pixel-by-pixel data at and around the spot, amplitude on the top, phase on the bottom. Note the great differences between phases in adjacent pixels.

Figure from CRISP program, Calidris Inc.
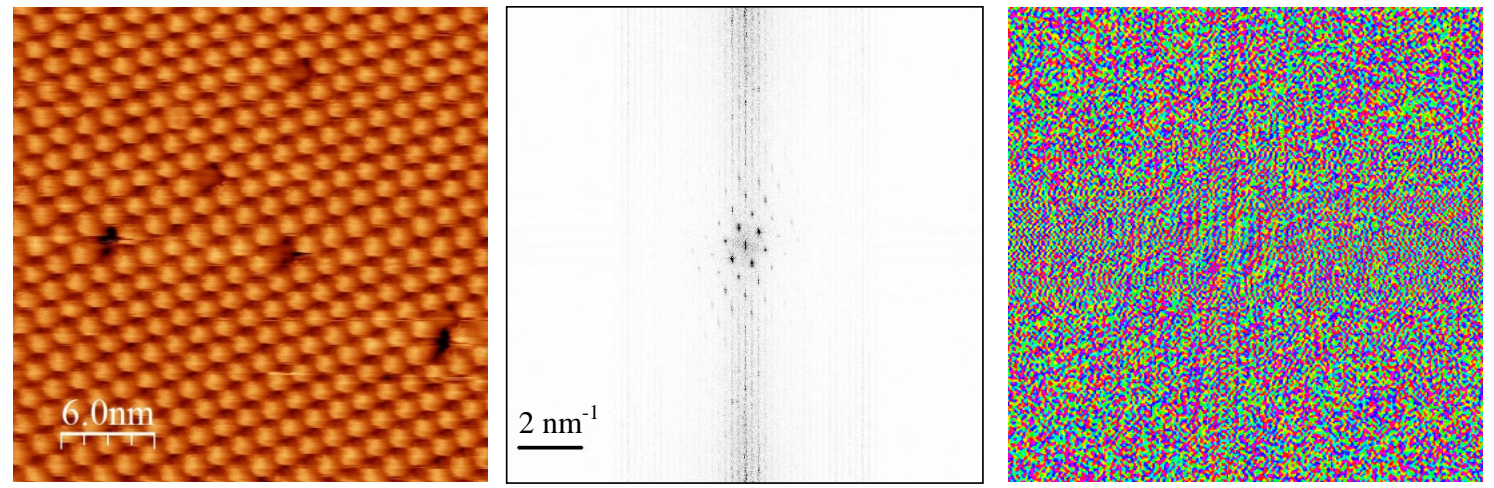

Figure 2.5. (left) STM image of $\mathrm{F}_{16} \mathrm{CoPc}$ on graphite, image analyzed with CIP in chapter 4; (center) plot of Fourier amplitude component squared of the same image; (right) plot of Fourier phase component of same image, false color corresponds to phase which ranges from -180 to 180 degrees. 
The second task is to index the spots, in other words to select the appropriate reciprocal lattice vectors. There are algorithms for automatic lattice detection - however the human eye is an excellent detector of patterns, and the user can label two particular spots with $H K$ indices and so select the lattice manually. Typically one chooses the two spots nearest to but not collinear with the central spot and assigns them as $(1,0)$ and $(0,1)$. Once this assignment has been made, the $H K$ indices of the remaining spots are compelled and the lattice parameters for the reciprocal lattice $\left(a^{*}, b^{*}\right.$, and $\left.\gamma^{*}\right)$ are determined.

Each spot now has six pieces of information associated with it: its $x^{*}-y^{*}$ location in reciprocal space, its $H K$ index, its amplitude component and its phase component. These last two components are the raw material for CIP.

The determination of these lattice parameters also determines the type of lattice. An object with two-dimensional periodicity can be organized in one of only five possible two-dimensional lattices. Figure 2.6 shows the four primitive lattices as well as one centered lattice. 


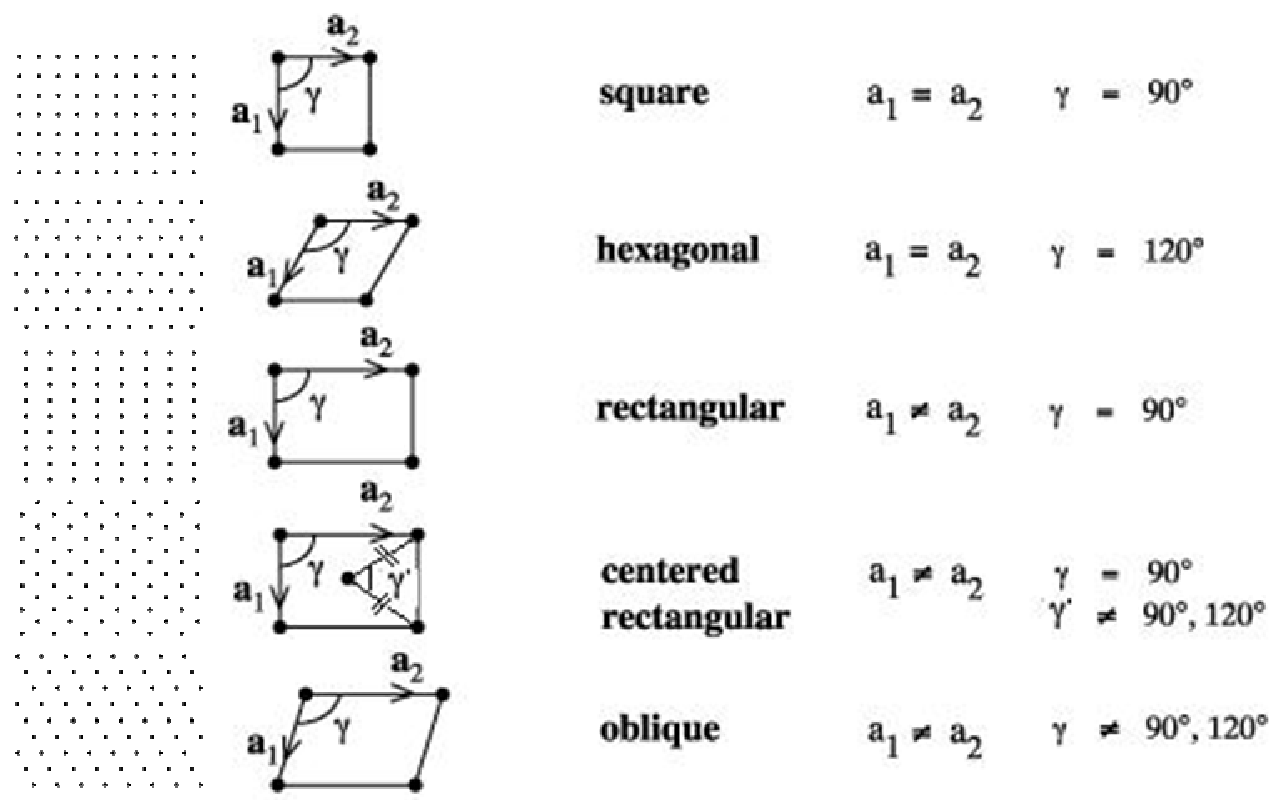

Figure 2.6. The five two-dimensional Bravais lattices. The square, hexagonal, rectangular and oblique lattices are primitive. The centered rectangular lattice is a centered lattice, and can also be considered a rhombic (primitive) lattice, in which case the basis vectors are the two equal line segments marked with double hash marks. No other lattices are possible in two dimensions.

This lattice determination is made while looking at the reciprocal lattice but it applies to both the reciprocal lattice and the image (real space) lattice. The image will likely have noise, which if random does not interfere with the symmetry, but may also have distortions in the $\mathrm{x}-\mathrm{y}$ directions that can make both the real and reciprocal lattices appear to have less symmetry than the actual sample possesses.

3. Make a symmetry assumption. As stated before, there are only 16 possible higher symmetry plane groups (groups that possess point symmetry elements higher than the identity, i.e. not $p l$ ) to which a two dimensional periodic object can belong. Each one of these groups constrains the relationships between the Fourier coefficients, 
both amplitude and phase, of various spots in various ways. In some cases there is a null constraint. For instance in the case of $p 2$ symmetry, there is no constraint on the amplitudes but all phases must be 0 or 180 degrees. As an example of a highly symmetric group, in the case of p6mm not only do the phases have to be 0 or 180 degrees, but both the phases and the amplitudes of any spot at $(H K)$ must equal the phases and amplitudes of spots at $(K-H-K),(-H-K H),(K H),(H-H-K),(-H-K K)$, as well as $(-H,-K)$.

The sample, if periodic, must belong to one of these groups. Each group is tested in turn, although for computational efficiency if the reciprocal axes are close to 90 degrees the hexagonal groups $(p 3, p 3 m 1, p 31 m, p 6, p 6 m m)$ are not tested, while if the axes are close to 60 degrees the square $(p 4, p 4 m m, p 4 g m)$ and rectangular $(p m, p g, c m$, p2mm, $p 2 m g, p 2 g g, c 2 m m)$ groups are not tested.

3.1 Find the origin. Given the lattice parameters, a unit cell is implicitly defined in the reciprocal lattice. This defines the size of the unit cell in the image. However, the correct origin, about which the plane symmetry group constraints apply to the Fourier coefficients, must be located in real space (the image). The origin may be at specific points or along certain mirror or glide lines. Each of these possible locations is tested, and for each location the "residuals" of the amplitude and phase components are calculated for the Fourier components.

The residuals are an objective measure of difference between the symmetry of the selected plane group and the actual data. 


$$
A_{\text {res }}=\frac{\sum_{H, K}|| A_{o b s}(H, K)|-| A_{\text {sym }}(H, K)||}{\sum_{H, K}\left|A_{o b s}(H, K)\right|}
$$

is the formula for amplitude residuals and

$$
\varphi_{\text {res }}=\frac{\sum_{H, K} w(H, K) \cdot\left|\varphi_{\text {obs }}(H, K)-\varphi_{\text {sym }}(H, K)\right|}{\sum_{H, K} w(H, K)}
$$

is the formula for phase residuals.

In each case, the summation can be taken over every $H, K$ value in reciprocal space for which a nonzero coefficient exists; often coefficients with small amplitudes are disregarded. A lower limit can be placed on the amplitude component, with coefficients having a smaller amplitude left out of the sum. This is for ease of computation and because low-amplitude coefficients contribute very little to the visual image.

The $o b s$ subscript refers to value from the DFT calculation and the sym subscript is the predicted coefficient once an origin has been selected. In the case of the amplitude calculation, the formula expresses the residual as a simple sum of absolute differences divided by the sum of the actual values. The phase calculation is a little more complex in that the difference values in the numerator are weighted by a factor $w$ that is usually the amplitude of that spot [6] and the denominator is the sum of those weighting factors (see equation 2.8). 
When all possible origins have been tested, the position with the overall lowest residual is selected as the origin of the unit cell. That position's residuals are stored and will be compared later against the residuals of the other plane groups as an indication of the likelihood of that symmetry being the correct one for the sample.

To summarize, these important quantities (residuals) are the difference between the observed amplitudes and phases taken from the calculated reciprocal lattice array and the amplitudes and phases that would exist if the origin of the assumed plane group were set at the assumed location. This set of difference calculations is repeated for each plane group that is being tested; the results are then compared.

3.2 Choose a group. Further work is necessary to find totally objective criteria for this decision. The determination of a non-subjective means of selection of the correct plane group is in progress. Although residuals are a reproducible (within the same algorithm) quantification of difference, it is not sufficient to merely choose the plane group with the smallest residual.

It is tempting to pick the group with the lowest residual and assign that group as the best match (and in many cases of course it is). Clearly low residuals for a particular group indicate that the symmetry of the image closely matches the symmetry of that group. However in many cases the residuals for several groups are roughly similar and the best pick is not the absolute lowest, but the group from this collection that has the highest multiplicity, if it is a subgroup of the one with the lower multiplicity. 
As an example, every image with $p 3 m 1$ symmetry also has $p 3$ symmetry, but the converse it not true; $p 3$ does not imply $p 3 m 1$. Thus any object that actually possesses p3ml symmetry will have low residuals for $p 3 m 1$ but will also have low $p 3$ residuals. It may happen that the $p 3$ residuals are actually lower than those for $p 3 m 1$ by a small amount; but $p 3 m 1$ is the correct choice nonetheless.

Some weighting scheme that accounts for the multiplicity of the various groups is necessary. One approach would be that of Kanatani [7] who was commenting on a method of quantifying two-dimensional symmetry put forth by Zabrodsky [8]. Although Zabrodsky's model has more in common with point symmetry than the crystallographic symmetry that this thesis addresses, it has the same problem with what Kanatani describes as the "hierarchy" of symmetries, in which one symmetry is a subset of the other, which can lead to misidentification of the best symmetry.

Kanatani examines the case of fitting irregular polygons to polygons with various levels of symmetry and suggests a calculation involving degrees of freedom, which vary with the various levels of symmetry. His model calls on the geometric Akaike information criterion (AIC) and appears to give a theoretical justification and a quantifiable measure for when to select a higher level of symmetry than raw data would immediately suggest.

The program CRISP makes the plane group selection automatically (although it can be overridden by the user). As the program is designed for the TEM community its 
choices are optimized for the types of symmetry an electron crystallographer is likely to encounter. This is not necessarily optimum for SPM users.

4. Enforce the selected symmetry. When a decision has been made as to which plane group is the best match, the Fourier coefficients (which will be approximately correct by virtue of the match) are adjusted. Amplitudes that are supposed to be equal for symmetry reasons are forced equal; phases that are supposed to be equal or 180 degrees out of phase are forced into this configuration; and a new, reconstructed unit cell using these corrected Fourier coefficients is generated in real space.

\subsection{Imaging}

Every imaging instrument, whether an optical telescope or an electron microscope, is intended to observe internal detail of an object. Real objects exist in three dimensions and their detail can be represented by a three-dimensional density function. This density function is often of mass but could be charge, magnetic dipole moment, or whatever physical quantity the imaging instrument can measure. The internal detail that is available to an imaging instrument is called contrast and can be represented by a two-dimensional density function.

The simplest object, a point source, can be represented as a delta function (thus having no internal detail at all). Any real world object with detail, observed at visible wavelengths, has a flux of visible light that varies across the object (although a star can be treated as a point source in many astronomical contexts). Similarly a TEM sample, from the point of view of the instrument, has a field of electrostatic potential energy 
from the atoms in the sample, which varies across the sample, providing contrast when imaged with electrons that are scattered at this potential. Crystalline samples in particular have periodic variations in contrast if viewed with capable instruments and because of the periodicity in real space, their images can be processed in particularly useful ways.

An ideal instrument would perform an exact linear transformation of the density function of the object to an image, changing only the Cartesian coordinates according to the magnification of the instrument.

$$
i(M x, M y)=o(x, y)
$$

where $M$ is magnification.

In other words, an ideal instrument maps a point to a point. A real instrument maps a point to an extended area. The transformation from object plane to image plane is described by the point spread function, which defines how one point in the object is spread out onto the image. Consider a point source in the object plane that can be described as a delta function $\delta(x, y)$. Using the notation from van Dyck [9],

$$
\hat{l}[\delta(x, y)]=p(x, y)
$$

where the operator $\hat{l}[\delta(x, y)]=p(x, y)$ transforms the infinitely sharp object $\delta$ into a blurred image $p$. Any real instrument maps $\delta$ onto a smeared area of the image plane. We will assume for now that $p$ is itself radially symmetric (i.e. it transforms a point into a circular disk whose density varies only with distance from the center) and constant 
across the object plane (translation invariant); in reality certain aberrations, for example coma, show that with many types of optical or electron instrument $p$ becomes asymmetric with distance from the center of the object. The point spread function is usually associated with optical and electron imaging instruments, but the concept applies to SPMs as well. We will consider the point spread function of SPMs as virtually translation invariant given the design of their imaging process and demonstrate with an example.

Given that any extended object is made up of many points located at various places in the object plane, any point in the image plane of a real instrument may contain contributions from different points in the object plane. If we call the density function of the extended object o(r) where $\mathbf{r}$ is a vector in two dimensions, again following van Dyck's treatment, the entire object is a weighted integral of delta functions:

$$
o(r)=\int o\left(r^{\prime}\right) \delta\left(r-r^{\prime}\right) d r^{\prime}
$$

and the image is

$$
i(r)=\int o\left(r^{\prime}\right) \hat{l}\left[\delta\left(r-r^{\prime}\right)\right] d r^{\prime}=\int o\left(r^{\prime}\right) p\left(r-r^{\prime}\right) d r^{\prime}
$$

which is the mathematical definition of the convolution $o \otimes p$; intuitively, since each point in the image is formed from the point spread function as it is applied to many points in the object, the total image, expressed as a function of two variables, is the convolution of the object density function and the point spread function. Note that in the case of an ideal instrument, the point spread function reverts to a delta function and therefore the image equals the object, as it must. 
Let $o(\boldsymbol{r})$ represent the object, $p(\boldsymbol{r})$ the point spread function, and $i(\boldsymbol{r})$ the image. The convolution theorem states that given

$i(\boldsymbol{r})=o(\boldsymbol{r}) \otimes p(\boldsymbol{r})$

where $\otimes$ represents the convolution operator, then

$I\left(g^{*}\right)=O\left(g^{*}\right) P\left(g^{*}\right)$

where $I, O$, and $P$ are the Fourier transforms of their respective functions. The ability to calculate with products instead of integrals means that computationally it is in some ways easier to deal with these relationships in Fourier space than in real space (there are also other advantages of Fourier space to be discussed when dealing with periodic objects).

One complication is that unless the point spread function $p(\mathbf{r})=p(r, \theta)$ is radially symmetric, i.e. constant with respect to $\theta$, its Fourier transform $P$ will be complexvalued. To see that a radially symmetric function only has real components to its transform, consider that the Fourier transform

$$
F(H, K)=\int f(x, y) \exp (2 \pi i(x H+y K)) d x d y
$$

can be restated (using the Euler formula) as

$$
F(H, K)=\int_{\text {entire }- \text { plane }} f(x, y)[\cos (2 \pi(x H+y K))+i \sin (2 \pi(x H+y K))] d x d y
$$

Since a radially symmetric function has no $\theta$ dependence, points that are equidistant from the origin are equal. In particular, points equidistant from the origin 
that are 180 degrees apart must be equal. Thus $f(x, y)=f(-x,-y)$ and the integral can be taken over just one half of the plane as:

$$
\begin{aligned}
F(H, K)= & \int_{\text {half_plane }} f(x, y)[\cos (2 \pi(x H+y K))+\cos (2 \pi(-x H-y K))] d x d y \\
& +\int_{\text {half_plane }} f(x, y)[i \sin (2 \pi(x H+y K))+i \sin (2 \pi(-x H-y K))] d x d y
\end{aligned}
$$

Since the sine is an odd function, the imaginary terms drop out, leaving only the real cosine terms. This fact is analogous to the fact that centrosymmetric crystals have Fourier phases restricted to 0 or 180 degrees.

\subsection{Application of CIP to High-Resolution Transmission Electron Microscope Images}

The first application for CIP (and in fact the reason it was invented) was the

processing of high resolution transmission electron microscopy images of periodic samples.

When seeking HRTEM images, one finds that amplitude contrast images (masscontrast or diffraction contrast, i.e. bright-field and dark-field images) do not provide atomic-level resolution. A sample that is too thin exhibits little amplitude contrast; amplitude contrast in a thin sample has been compared [9] to imaging a glass plate of variable thickness in an optical microscope; but a sample thick enough to provide useful amplitude contrast also suffers from dynamic scattering that hampers resolution. 
The technique of choice for a crystalline sample is phase contrast imaging, in which the sample is very thin and the direct beam is combined with as many diffracted beams as possible. The diffracted beams have been phase-shifted according to the contrast transfer function $(\mathrm{CTF})$ and the resulting interference provides contrast in the image plane, depending upon the amount of defocus (a perfectly focused image has no contrast in this circumstance).

The resulting image is essentially a set of interference fringes, and visually does .represent a "picture" of the sample. The lattice fringes convey a strong visual impression that one is looking directly down the atomic planes. This is not correct. However, if the sample is periodic and symmetric, i.e. crystalline, CIP can extract information from the HRTEM image. Given a set of images taken from various angles the user can reconstruct the three-dimensional structure of the sample.

Any periodic and symmetric sample is a suitable target for CIP investigation. If the complete three-dimensional structure is already known in part, the image enhancement that CIP can provide even to a single periodic image can clarify that structure.

An example is the work of Oku [10]. He was seeking details of the atomic position of boron and dopant atoms in large highly regular arrangements thought to be made of icosohedral boron substructures connected to form buckyball-like structures. 
He took HRTEM images of these structures, seen below in Figure 2.7. Both images are highly periodic in two dimensions but have noise and variation in sample thickness. Both images are taken at nearly the Scherzer defocus and thus show electron potential directly. The $\mathrm{B}_{105} \mathrm{Al}_{2.6} \mathrm{Cu}_{1.8}$ image was taken with a $1250 \mathrm{kV}$ instrument with resolution of $0.12 \mathrm{~nm}$ and the $\mathrm{B}_{56} \mathrm{Y}$ image was taken with a $400 \mathrm{kV}$ instrument with a resolution of $0.17 \mathrm{~nm}$.

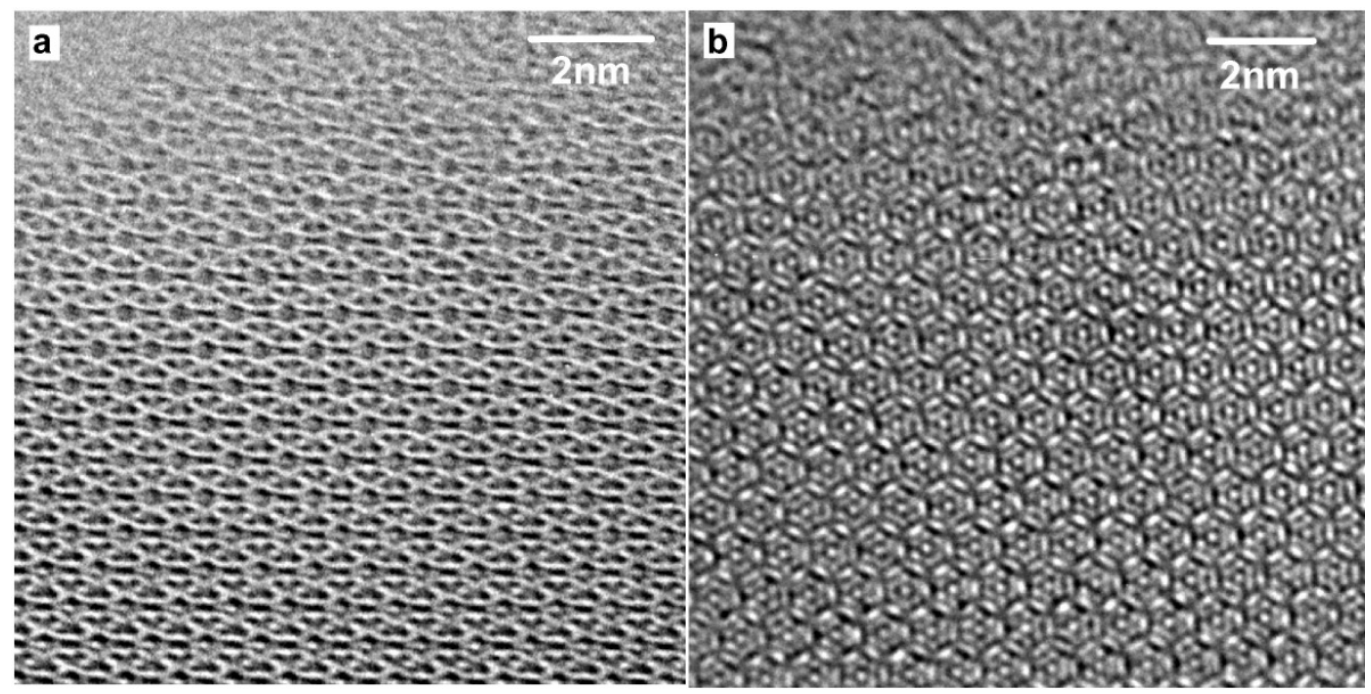

Figure 2.7. HRTEM images of (left) $\mathrm{B}_{105} \mathrm{Al}_{2.6} \mathrm{Cu}_{1.8}$ along [211] direction; (right) $\mathrm{B}_{56} \mathrm{Y}$ along [111] direction. [Oku]

Oku used CRISP to process the images. He applied standard CIP techniques and enforced $c 2 m m$ symmetry on the $\mathrm{B}_{105} \mathrm{Al}_{2.6} \mathrm{Cu}_{1.8}$ image, $p 6 m m$ symmetry on the $\mathrm{B}_{56} \mathrm{Y}$ image. The prior knowledge of the resolution limit of the microscopes enabled Oku to confidently disregard Fourier coefficients of an order greater than the resolution limit of the microscope, converted to reciprocal space. The noise reduction is clear in figure 2.8 , 
which is the $\mathrm{B}_{105} \mathrm{Al}_{2.6} \mathrm{Cu}_{1.8}$ image of figure 2.7 with a small area of $c 2 m m$-enforced image superimposed.

Oku's processed images are shown in figure 2.9. Superimposed upon the processed images are Oku's proposed locations for the boron structures. The location of the dopant atoms in the $\mathrm{B}_{105} \mathrm{Al}_{2.6} \mathrm{Cu}_{1.8}$ on the left in the figure is particularly clear because of CIP; they are barely visible in figure 2.7 (left)

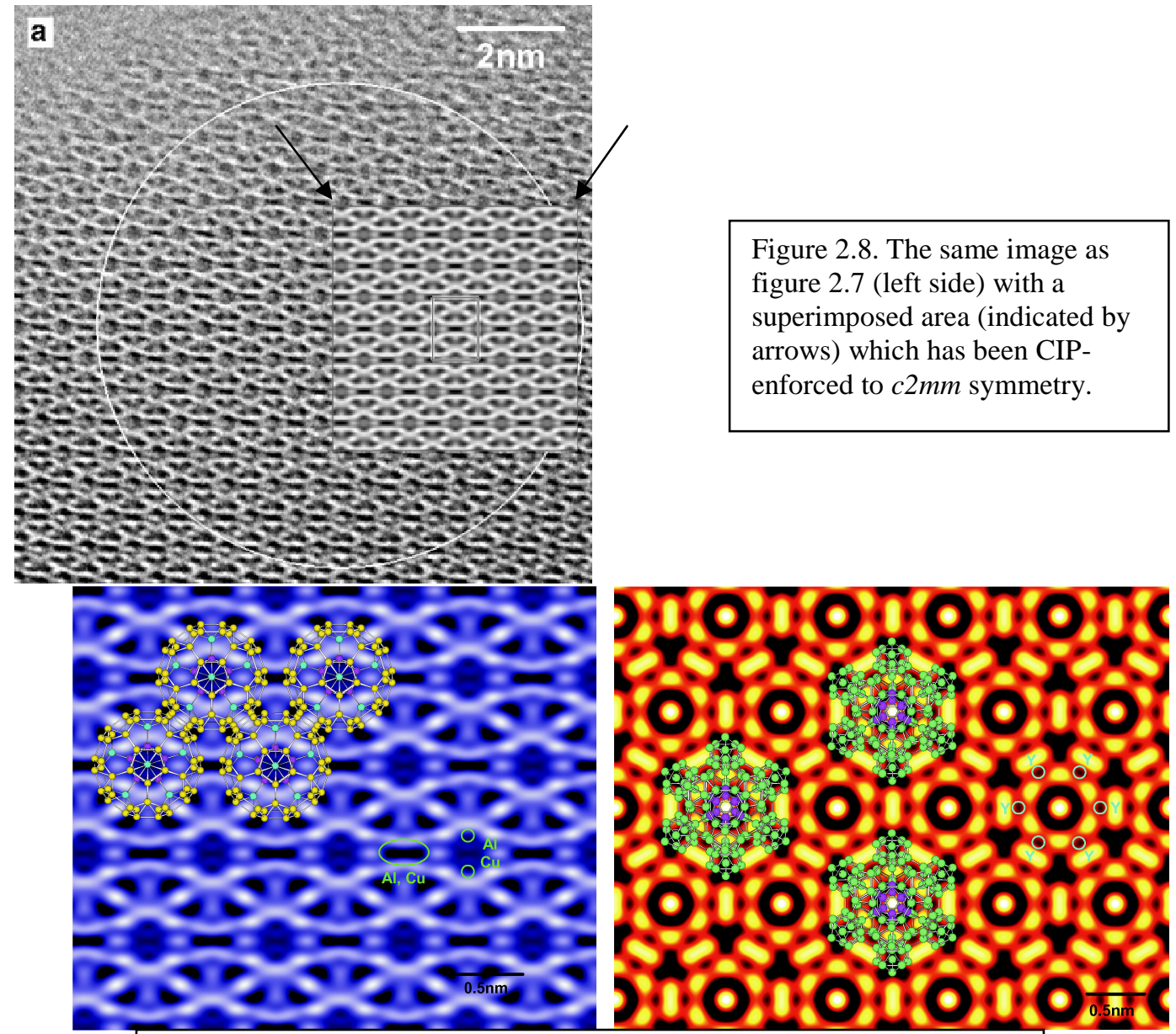

Figure 2.9. CIP-enforced images of samples in Figure 2.7, with proposed dopant molecule locations illustrated. (left) $c 2 m m$ enforced $\mathrm{B}_{105} \mathrm{Al}_{2.6} \mathrm{Cu}_{1.8}$ (right) p6mm enforced $\mathrm{B}_{56} \mathrm{Y}$. The color schemes (blue on the left, orange on the right) are for visual clarity; these are grayscale images. 
Oku also calculated simulated images of these specimens, shown in figure 2.10. They closely resemble the CIP-enforced HRTEM images, including the doping atoms (although the $\mathrm{B}_{105} \mathrm{Al}_{2.6} \mathrm{Cu}_{1.8}$ real and CIP-enforced image contains some elongation along the $\mathrm{x}$-axis not present in the calculated image), supporting the likelihood that the CIP processed images represent something close to physical reality.

The application of CIP to these HRTEM images of three-dimensional objects is analogous to the intended application of CIP to SPM images. The single 2D projection of this 3D sample is the sole input to the CIP procedure. The procedure benefited from the prior knowledge of resolution limit and some knowledge of the sample. Similarly CIP can be usefully applied to SPM images with some prior knowledge.
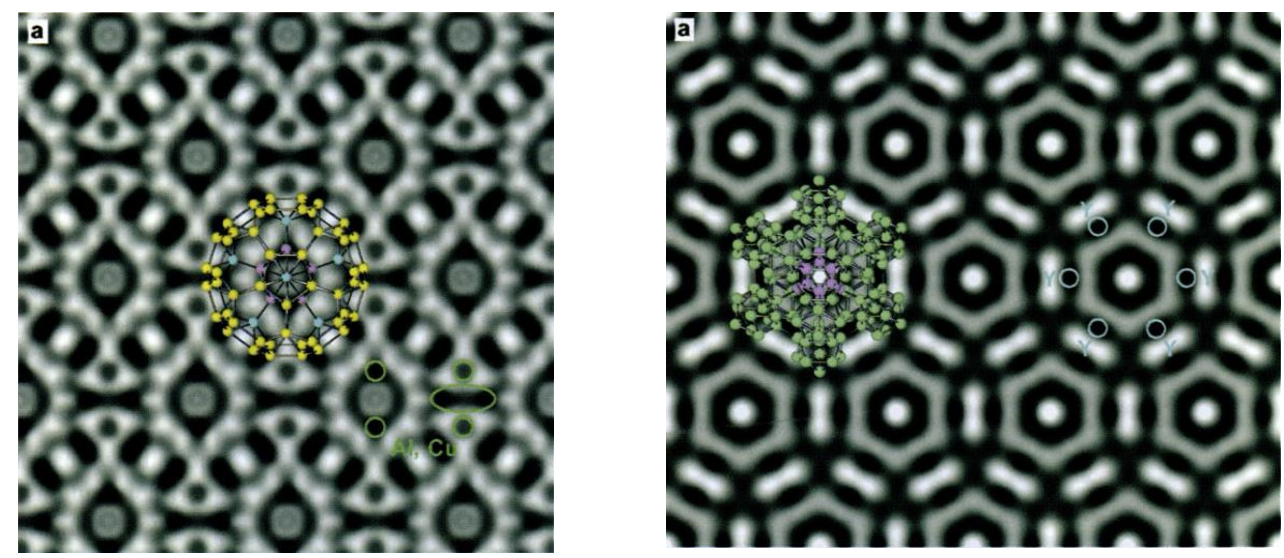

Figure 2.10. Simulated images of (left) $\mathrm{B}_{105} \mathrm{Al}_{2.6} \mathrm{Cu}_{1.8}$ (right) $\mathrm{B}_{56} \mathrm{Y}$, and dopants. 


\subsection{CIP applications to Scanning Probe Microscope images}

Scanning probe microscopy (SPM) is the collective term for a completely different design of imaging instrument whose two main variants are scanning tunneling microscopy (STM) and atomic force microscopy (AFM).

The idea of applying Fourier filtering to SPM images is not new. In 1987 Park and Quate filtered noise out of the Fourier transforms of their images as a means of improving image quality [11]. However they did not enforce any crystallographic restrictions on the Fourier coefficients.

The principle behind these instruments appears very simple - a probe is made to approach the surface of the sample, almost touching it, and is then scanned across the surface in a raster pattern while the response of the probe is recorded. Not only is the engineering that enables this to take place at the atomic level demanding, the very definition of "touch" has to be examined in some detail. The probe itself is not a passive instrument. The data observed comes from interactions between relatively few atoms in the sample and relatively few atoms in the probe (in the case of STM), so the nature of the probe is inextricably part of the observing process.

The physics behind STM compels tunneling current (the quantity being measured) to travel almost completely through one atom in the tip, the one closest to the sample. STM is able to image atomic level detail, including defects, for a conducting sample. The requirement that the sample be conducting is a significant limitation. 
AFM is able to image nonconducting samples because the parameter being measured is minute differences in force, rather than differences in tunneling current. There are variants of AFM including magnetic force microscopy (MFM) and piezo force microscopy (PFM) that operate similarly but are measuring minute differences in other types of force. The most general AFM instrument measures interatomic forces between the tip and the sample. AFM tips need not be conducting; typical tips are silicon or silicon nitride.

STM requires surfaces to be atomically flat with occasional steps and for most samples requires ultra-high vacuum for atomic resolution (graphite is an exception and can be resolved in ambient conditions). AFM can observe rougher surfaces. AFM can be operated in contact mode, which was the condition of early experiments. Better resolution is found with non-contact mode, in which the cantilever carrying the tip is deliberately vibrated at a frequency at or near its resonant frequency. The tip is scanned at constant height above the sample and interatomic forces acting on the tip slightly change the phase and/or resonant frequency of the cantilever. Either the amplitude or frequency of the oscillating cantilever is the output data.

A recent paper by L. Gross [12] describes a detailed observation of pentacene using frequency modulated AFM. This is unusual in that the molecule is not part of a crystal lattice, it is alone on the substrate. In the past attempts at imaging single molecules resulted in unacceptable distortion to the molecule being observed. Gross prepared tips by picking up a single $\mathrm{CO}$ molecule that adhered to the apex, with the 
oxygen atom protruding as the actual tip and found under cold UHV conditions that he could image the pentacene clearly, including the hydrogens.

\subsubsection{Scanning Tunneling Microscopy}

The STM was invented by Gerd Binnig and Heinrich Rohrer in 1982 (for which they shared the 1986 Nobel Prize in Physics with Ernst Ruska, the inventor of the transmission electron microscope five decades earlier).

The instrument consists of a tip, generally made out of a transition metal and as sharp as possible (more on this below), bonded to three orthogonal piezoelectric transducers [13] two of which respond to applied voltages by changing the location of the tip so as to scan in a raster pattern in the $\mathrm{x}$ - and $\mathrm{y}$ - directions across an electrically conductive sample.

The third transducer, in the z-direction plays a somewhat different role. A mechanical linkage brings the tip into close approach to the sample and a bias voltage on the order of \pm 1 volt is applied to the tip with respect to the sample. The tip is slowly

\footnotetext{
Figure 2.11 diagram of an STM.

Creative Commons

- Wikipedia
}

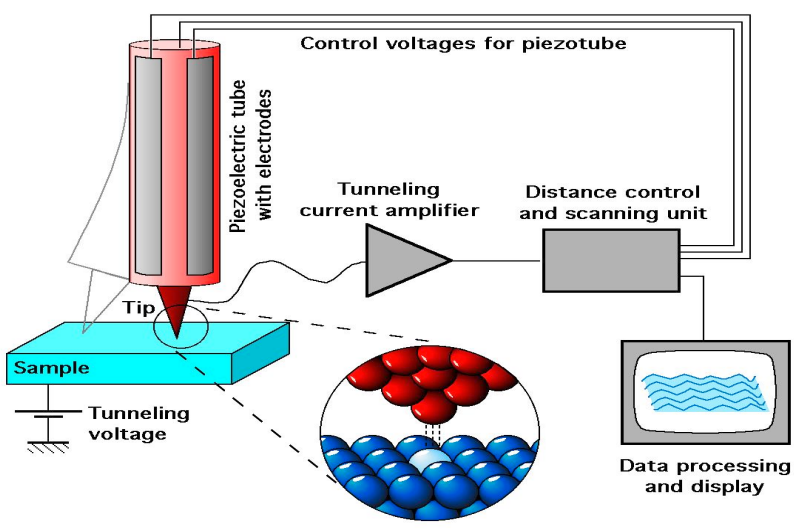


lowered under computer control until, when the distance between tip and sample is a few atomic diameters, a current begins to flow between tip and sample. Figure 2.11 is a block diagram of a typical STM.

This current flows because of quantum mechanical tunneling between tip and sample across the potential barrier of the gap (which may be vacuum, air or other gas, or in some cases liquid). If the work function $\Phi$ is large compared to the absolute value of bias voltage then the barrier is essentially rectangular (see Fig. 2.12) and the quantum mechanical solution for current is relatively simple.

$$
I=I_{0} e^{-2 \kappa_{t} z}
$$

where $z$ is the distance from the sample and $\kappa_{t}=(2 m \Phi)^{1 / 2} / \hbar$. The strong distance dependence means the atom at the tip closest to the sample carries most of the tunneling current, even if the tip is relatively blunt, because at least one atom is likely to protrude and thus contain most of the tunneling current [14]. There is relatively little interaction between other atoms in the tip and more distant atoms in the sample.

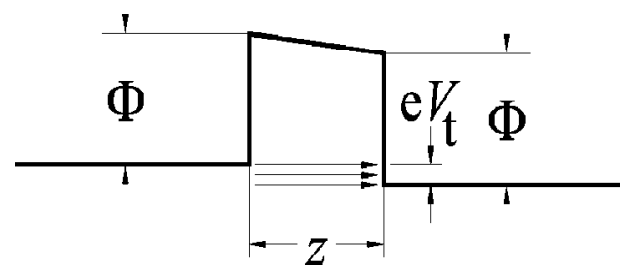

Fig. 2.12

If $\Phi \gg\left|e_{t}\right|$ the trapezoidal barrier can be approximated as a rectangle from Giessbl 2003

The value of the current is fed back into the z-piezo as negative feedback. For imaging purposes, there are two modes of operation for an STM. Constant current mode uses the feedback information to move the tip vertically so as to maintain constant 
current and uses the tip height as data output. Constant height mode, or topographic mode, attempts to keep the tip at the same position and uses the varying current value as the data output. Constant height mode is faster but requires a flatter surface, or there is a risk of tip crash.

In either case the tunneling current depends upon the local density of states (LDOS) of the sample at the Fermi level so the image is a real-space representation of the electrons on the surface of the sample.

\subsubsection{Atomic Force Microscopy}

A significant shortcoming of STM is the requirement that the sample be electrically conductive. A later instrument, the atomic force microscope (figure 2.13),

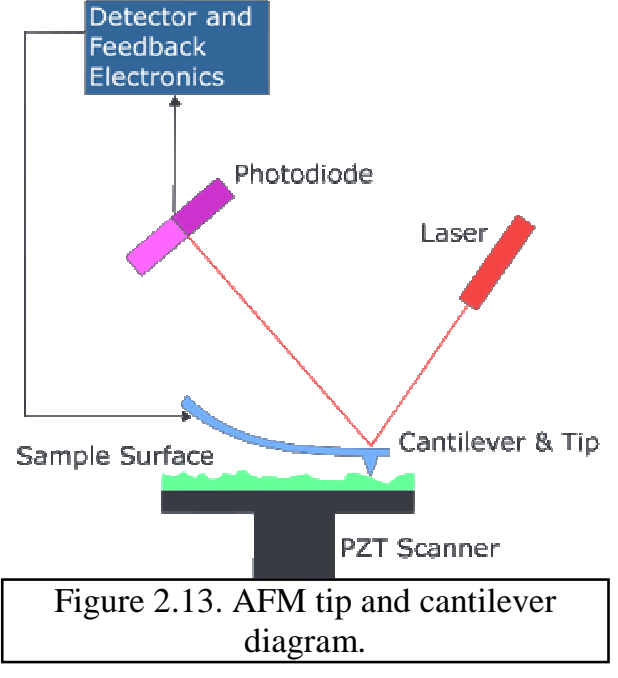
does not have this disadvantage.

The AFM was invented in 1986 by Binnig, Quate and Gerber [15]. It is a scanning instrument with a tip whose $x-y$ motion is controlled by piezoelectric ceramics and rastered across the sample in a fashion similar to an STM.

The tip is either bonded to the end of a cantilever, or the tip and cantilever are an integrated piece of material. Various methods of measuring the cantilever position have been tried. Commonly today a laser is focused on the back of the cantilever, and as the 
probe moves over the sample, motion of the tip/cantilever is translated into deflection of the reflected laser light that is observed by a set of photodiodes. The cantilever is designed to be rigid in two directions and soft in the third direction (usually the $z$ direction).

The physical operation of the instrument is similar to an STM in that a mechanical link brings the probe close to the sample in the z-direction, then piezos bring the tip close enough to begin sensing atomic forces. Now, however, the instrument is measuring the minute interatomic forces instead of measuring a current. At long distances these forces are attractive van der Waals forces, which increase as distance decreases. As the distance decreases beyond an equilibrium point, Pauli repulsion begins to repel the atoms, increasing at appreciable rate. An early but still useful model is the Lennard-Jones approximation for interatomic potential energy:

$$
V(r)=4 \varepsilon\left[\left(\frac{\sigma}{r}\right)^{12}-\left(\frac{\sigma}{z}\right)^{6}\right]
$$

where $\varepsilon$ is the depth of the potential well, $\sigma$ is the distance at which $V$ (the potential energy) is zero and $r$ is separation between the atoms. This model was devised in 1924 by John Lennard-Jones and is an easily calculated approximation to interatomic forces. The force is the space derivative of this curve.

The instrument can be operated with the cantilever static (DC mode) which is usually a "contact" mode; this means the tip is close enough to the sample that there is a repulsive force between the tip and sample [16]. The spring constant of the cantilever 
should be less than the spring constant of the chemical bonds of both the sample and the tip (if greater the tip is likely to rearrange atoms in either the tip or the sample) [17]. The resonant frequency of the cantilever must also be substantially higher than the highest frequency expected in the input signal by the variation in the sample as it is scanned.

More commonly it is operated in an AC mode in which the cantilever operates at or near its resonant frequency. This is usually either a "noncontact" mode that leaves the tip further away from the sample, in range of attractive forces between tip and sample rather than repulsive; or "tapping" mode that periodically brings the tip close enough to the sample to experience repulsive forces. The cantilever must have a stiffer spring constant or risk being pulled into the sample. The absolute magnitude of the forces is less than contact mode, making measurement more challenging.

Unlike STM, in which the quantity being measured (tunneling current) is monotonic with respect to distance from the sample, the fact that interatomic force switches direction as the distance decreases means the AFM feedback mechanism must be more sophisticated. The slope of the AFM force vs. distance curve over most of the distance range is also less steep than the STM tunneling current vs. distance curve, which intrinsically makes it more difficult to extract signal from noise. Figure 2.14 shows the AFM datum, force, (in nanoNewtons) and the STM datum, tunneling current, (in nanoAmperes) as a function of distance in nanometers. 


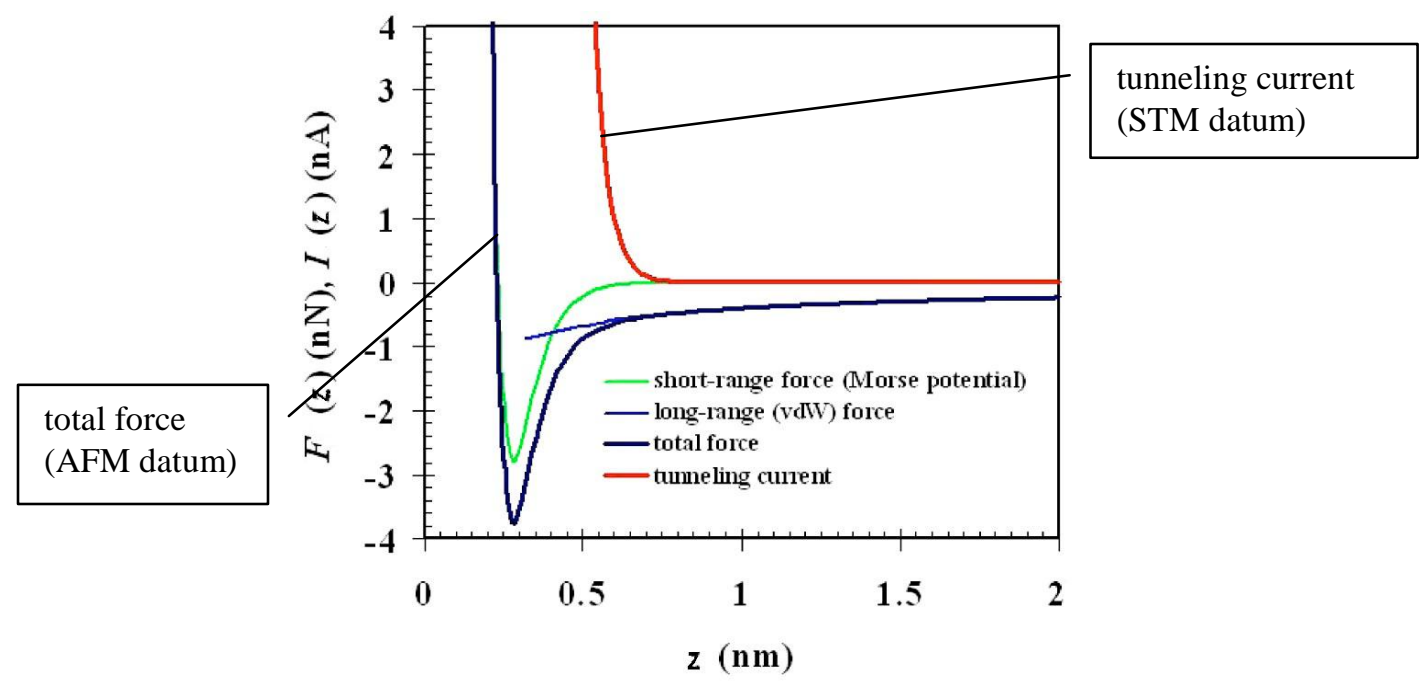

Fig. 2.14. Plot of tunneling current and force (typical values) as a function of distance $z$ between center of front atom of tip and plane defined by centers of atoms of surface layer. See text for formulas [Giessibl]

Tunneling current used in STM can be expressed as:

$$
I(z)=I_{0} e^{-2 \kappa_{t} z}
$$

where $\kappa_{t}$ is a constant and $z$ is distance from sample.

The force curve used in AFM is more complex and can be approximated by

$$
4 \varepsilon\left[\left(\frac{6 \sigma^{6}}{z^{7}}\right)-\left(\frac{12 \sigma^{12}}{z^{13}}\right)\right]
$$

the derivative of the Lennard-Jones potential.

Most tunneling current flows through a single atom in the STM tip because the current-vs.-distance curve is so steep. The interatomic force-vs.-distance curve is much less steep, thus more distant atoms in the tip and sample influence one another in an 
AFM. Consequently a much greater number of sample atom/tip atom pairs interact complicating the attempt to resolve atoms.

The quartz cantilever used in AFM is a high quality oscillator in the sense that its $\mathrm{Q}=($ energy stored in oscillator $) /($ energy lost per cycle) is very high. In other words, the cantilever is very lightly damped. Initially, investigators plotted the amplitude change ("amplitude modulation AFM") to see the topography, but because of the high Q of quartz, the amplitude of an oscillation changes very slowly in response to a stimulus. Frequency modulation AFM (FM-AFM) maintains a constant amplitude of cantilever vibration and varies the driving frequency; the driving frequency is the quantity plotted.

\subsection{Aberrations}

The ideal tip for general SPM observation would be a needle with one atom at the end. To the extent that this ideal is not reached, the fidelity of the image is suspect.

In crude terms, the intent of an SPM observation is measurement of the topography of a sample by "pressing" against it with a sharp tip and scanning that tip across the sample. (The actual definition of "pressing" depends upon the type of SPM. If an STM, "contact" begins when the tip is close enough to the sample that measurable tunneling current begins to flow, 0.4 to 0.7 nanometers. The tip of an AFM operating in noncontact mode can be many nanometers from the sample.) If the sample in fact consists of a series of sharp tips and the SPM tip is irregularly shaped (rather than sharp), what is actually observed is convolution of the topography of the tip and the sample. 
A significant amount of investigation has been made into the exact nature of the influence of an irregular tip on an image $[18,19]$.

A significant source of error in SPM is that fact that the piezoelectric transducers that move the tip in the $\mathrm{x}-\mathrm{y}$ direction are subject to hysteresis and creep [20]. A perfect transducer exhibits a displacement exactly proportional to the applied voltage, with a linear relationship between voltages and displacements. A perfect transducer will always exhibit the same displacement for the same voltage.

A real transducer will not exactly return to the same displacement when the same voltage is applied. Since SPMs typically scan their samples in a rectangular raster, the hysteresis causes successive horizontal lines to start from slightly different points, skewing what was presumed to be a rectangular array into a parallelogram.

Creep occurs because there are remnant domains in a piezoelectric crystal that do not immediately respond to applied voltage but do so over time. This creates a lowfrequency error as the piezo quickly deflects most of its range in response to an applied voltage but then drifts slowly in the same direction until ultimate deflection is reached. For example, if the mode of the raster scan is that horizontal scan is rapid in the $\mathrm{x}$ direction and the vertical scan is slower in the y-direction, ideally the vertical deflection will be linear as the probe scans sideways quickly. Creep will cause the rate of that vertical deflection to change as the probe scans sideways, an error that will be repeated periodically once every horizontal scan. 


\section{CIP as applied to an SPM image}

\subsection{Fluorinated cobalt phthalocyanine}

The organic molecule shown in most of the images of this thesis is cobalt(II) 1,2,3,4,8,9,10,11,15,16,17,18,22,23,24,26-hexadecafluoro-29H,31H-phthalocyanine, or fluorinated cobalt phthalocyanine $\left(\mathrm{F}_{16} \mathrm{CoPc}\right)$. It is a derivative of phthalocyanine, which is a cyclic compound whose structural formula is shown in Figure 3.1. The phthalocyanine group is commonly abbreviated Pc in chemical formulas. The molecule itself is $\mathrm{H}_{2} \mathrm{Pc}$.

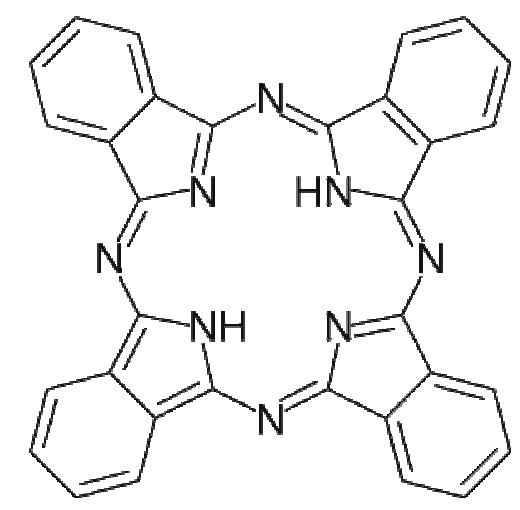

Figure 3.1. Phthalocyanine $\left(\mathrm{H}_{2} \mathrm{Pc}\right)$, structural formula.

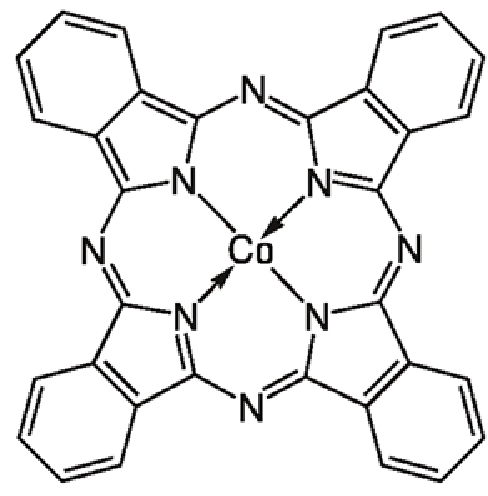

Figure 3.2. Cobalt phthalocyanine $(\mathrm{CoPc})$, structural formula.

The two hydrogen atoms in the central cavity of the Pc ring can be replaced by an atom of a transition metal forming a coordination complex (Figure 3.2). The resulting molecule is stable and poorly soluble in water. Most of these compounds are brightly colored and some have been used for decades as a dye. In particular the complex formed with copper is inexpensive as a bulk compound and is manufactured in large lots. The Sigma-Aldrich catalog has 88 entries for various phthalocyanine compounds. 
Many of the metal phthalocyanines also are $n$-channel semiconductors and are being investigated as components in thin-film organic field-effect transistors [21]. Replacing the 16 outer hydrogen atoms by halogen atoms increases both the carrier mobility and the stability of the compound in air [22]. Figure 3.3 is a sketch of $\mathrm{F}_{16} \mathrm{CoPc}$.

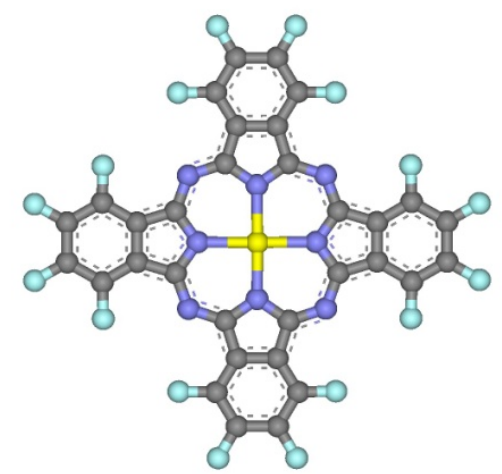

Figure 3.3. Sketch of fluorinated cobalt phthalocyanine. The outside circles (light blue if image is color) represent fluorine atoms. They have replaced the hydrogen atoms that are normally in this location.

Thin films of halogenated metal phthalocyanines have been observed to form monolayers on silver and graphite. There is some evidence that at lower temperatures these monolayers consist of molecules stacked partially or wholly on-end, whereas at room temperature the plane of the molecule appears to be parallel to the substrate [23].

The images shown in this thesis, with one exception, appear consistent with the flat orientation. The exception is the image is that called M16, whose Fourier coefficients imply at least the possibility that the molecule is oriented partially on end.

\subsubsection{Possible orientation of molecule on M16}

Although it does not devalue the worth of M16 as a calibration standard (which is based on the HOPG Fourier coefficients predominating), one can also make an observation about the symmetry of the image which (per conversations with Prof. Moeck at Portland State) leads to a suggested orientation of the $\mathrm{F}_{16} \mathrm{CoPc}$ molecule on 
the substrate: that it may be standing at least partially on end. This has been observed at low temperatures for copper phthalocyanine thin films [23].

By comparing the large number of Fourier coefficients in M16 to the few coefficients of a pure graphite image, it is clear that some trace of the organic molecule is present in the image. However if we choose the reciprocal lattice axes as seen in figure 3.4 , with an angle between them of $63.7^{\circ}$, it is clear that this array cannot have p4mm symmetry, which is what would be expected for a $\mathrm{F}_{16} \mathrm{CoPc}$ molecule that lies flat on an HOPG substrate. This in fact is how we will analyze this image later in this thesis, by concentrating on the HOPG substrate that is manifesting itself with these axes.

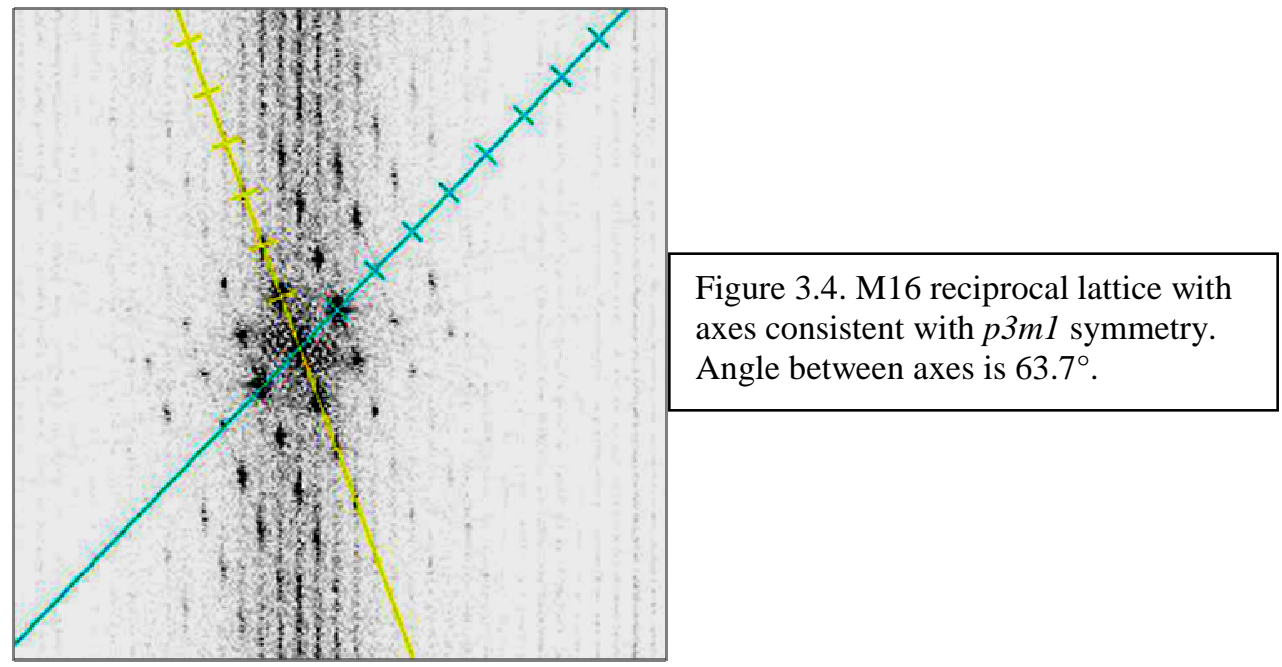

However, if the molecule were aligned with the underlying HOPG but partially turned on its end, three of the four mirror lines of the molecule's $4 \mathrm{~mm}$ point symmetry would be broken while one may remain intact. One would then expect to observe the plml and $p 11 \mathrm{~m}$ settings to have differing residuals, as well as the two settings of $p g$ and $\mathrm{cm}$. 
If we choose a set of rectangular axes as shown in figure 3.5, we find this to be the case as shown in figure 3.6, which shows the residuals for M16 as well as a 1.5 unit cell reconstruction with symmetry $p 1 m 1$. Note that for those plane groups with differing settings in the $x$ and $y$ direction $(\mathrm{pm}, \mathrm{pg}, \mathrm{cm})$ the residuals are in fact much lower for those plane groups with settings in the $y$ direction rather than the $x$ direction. The reconstructed image with $c 1 m 1$ enforced does show a definite two-lobed structure.

It is also interesting that the image M17 that was taken to the best of our knowledge at approximately the same time under similar experimental conditions and visually resembles M16 does not show the same two-lobed structure when clml is enforced.

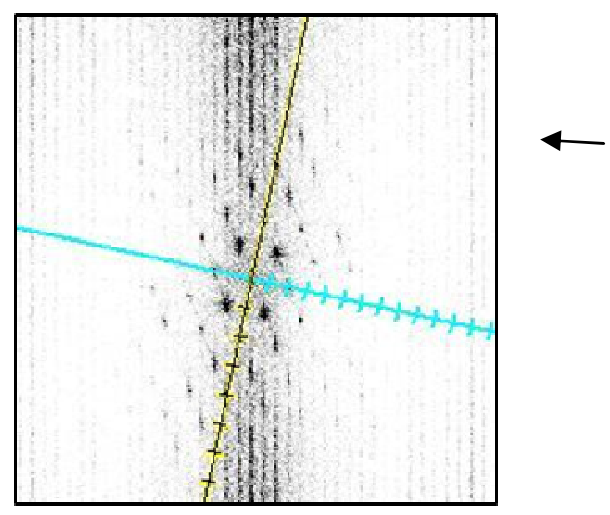

Figure 3.5. M16 reciprocal lattice with a different set of reciprocal axes, consistent with a centered unit cell. Angle between axes is $89.2^{\circ}$.

Figure 3.6. (left) Showing residuals for M16 using the centered axes in figure 3.5. (right) Contour plot of approximately 1.5 unit cells. Note two-lobed structure.
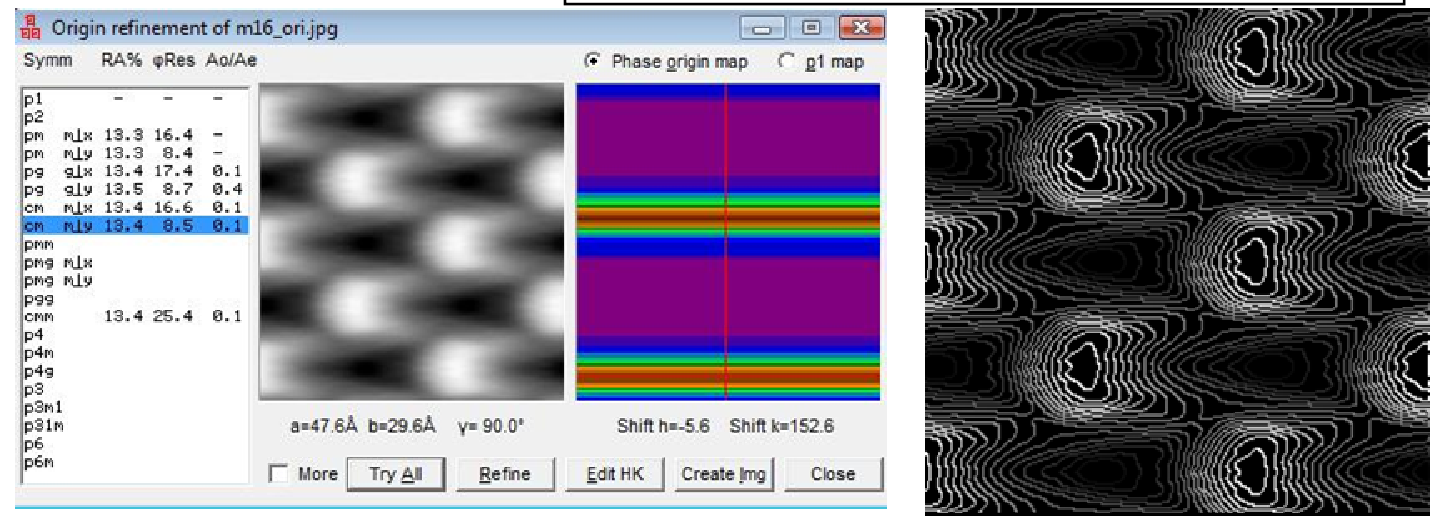


\subsection{Fluorinated cobalt phthalocyanine on HOPG}

We demonstrate here a specific application of CIP to an STM image. The image seen in figure 3.7 is this section's primary subject of analysis. It is a layer of $\mathrm{F}_{16} \mathrm{CoPc}$ molecules on highly-oriented pyrolytic graphite (HOPG) taken at the Technical University of Chemnitz. The sample was imaged under ultra high vacuum (UHV) conditions with a temperature of $20 \mathrm{~K}$. The sample is cooled with a liquid helium flow cryostat; however in this instrument the tip cannot be cooled and remains at room temperature [24]. The tip has a bias of +1.0 volt with respect to the sample.

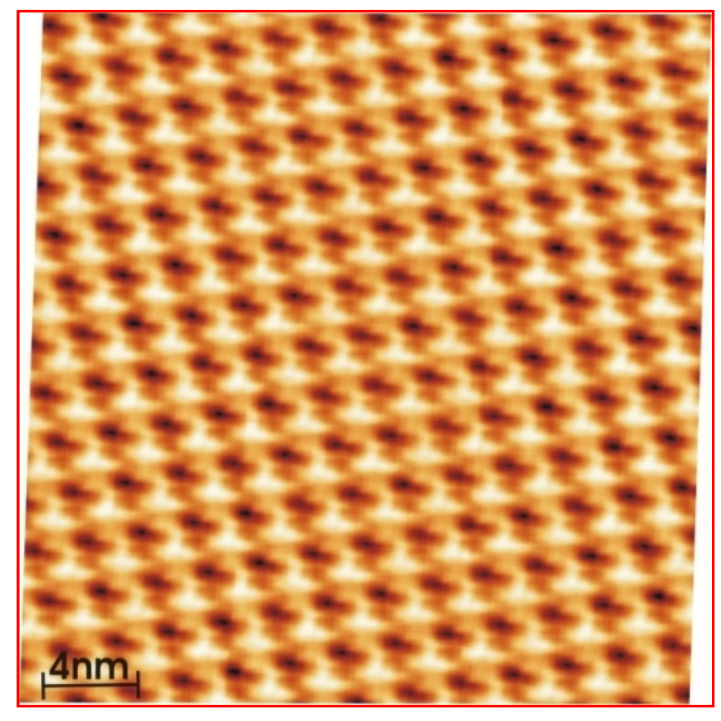

Fig 3.7 STM image of $\mathrm{F}_{16} \mathrm{CoPc}$ on HOPG; the cause of the slight clockwise skew of the image is unknown but CIP will remove it.

HOPG is well known to be a hexagonal crystal with $p 6 m m$ plane symmetry in the (0001) orientation, which is visibly not the configuration of this image. It is also well known that STM images of HOPG have $p 3 m 1$ symmetry [25]. This is because of the way layers of graphite nest on top of one another. Alternating carbon atoms in each hexagonal ring are either on top of another atom ( $\alpha$ atoms) or on top of a vacancy ( $\beta$ 
atoms). The existence or absence of an underlying atom changes the density of states for electrons in the top layer. Since the STM current depends upon the local density of states (LDOS), alternating carbon atoms have large differences in current.

For the purposes of comparison, figure 3.8 is an image of pure HOPG, without any organic molecule layer, taken at the Technical University of Chemnitz. An initial CIP analysis of this image shown in Figure 3.9 shows no high-order Fourier coefficients and suggests $p 3 m 1$ symmetry. Note the right half of Figure 3.9, which is a list of those Fourier coefficients above an amplitude cutoff and shows only six coefficients. (The

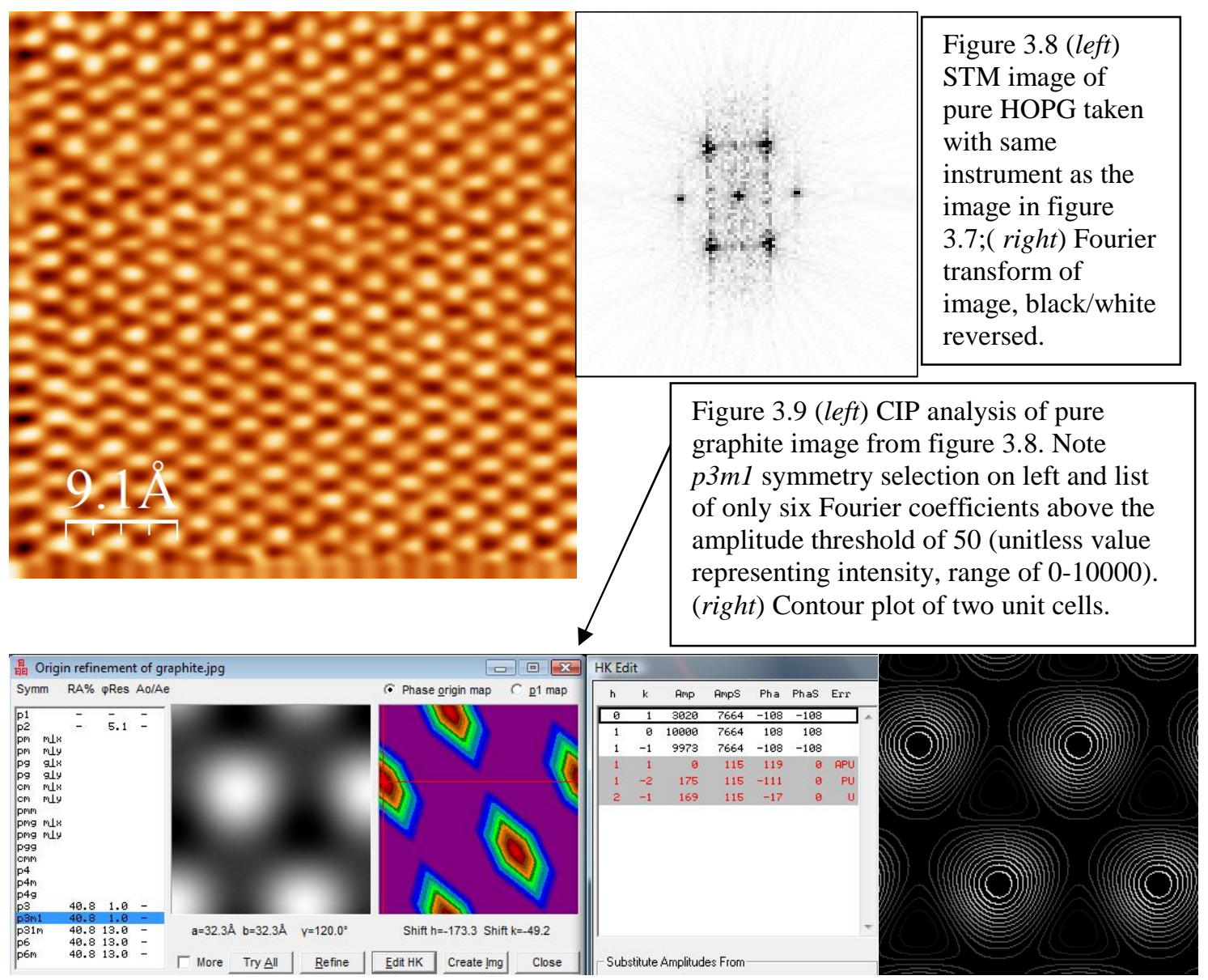


cutoff in this case is a value of 50 on a dynamic range of 0 to 10,000 for intensity). This is far fewer than the coefficients observed when the image in Figure 3.7 is analyzed with CIP with the same amplitude cutoff; that image has between 53 and 56 Fourier coefficients, depending upon exactly which location in the image is analyzed, indicating the presence of something other than graphite in that image.

\subsection{Possible orientation of the molecule}

The fact that one has prior knowledge of the molecule forming the layer is a significant clue that allows one to suggest the particular type of symmetry to enforce upon the image with a high likelihood of representing the physical reality. This is especially true in this case because the molecule is known to interact weakly with an HOPG substrate. This potentially allows the recovery of a significant portion of lost information. This information recovery is of course only as good as the prior knowledge and does not preclude the possibility that the molecule has elected an unusual alignment on the substrate, or that the molecule is significantly deformed. But presuming that this is not the case, we observe first that the $\mathrm{F}_{16} \mathrm{CoPc}$ molecule alone has two dimensional $4 \mathrm{~mm}$ point symmetry if lying flat on a substrate (see figure 3.3 ); that is to say, it can be rotated 90 degrees without change and can be reflected through any of the four mirror lines (horizontal, vertical, and two diagonal) without change. These mirror lines are the two-dimensional projection (projected when lying flat) of the mirror planes that exist in the three-dimensional molecule. Since the visual translational symmetry of Figure 3.7 is virtually square, it suggests that the molecules are aligned in a square array. However 
the repeating motif of the image bears little resemblance to the molecule. It is likely [24] that this is the result of a blunt tip containing multiple mini-tips. The idea here is to treat that tip as a "black box" and see what information can be recovered from the image.

If the $\mathrm{F}_{16} \mathrm{CoPc}$ molecule is not distorted on the HOPG substrate, the twodimensional arrangement of the organic molecule layer could potentially have a symmetry as high as $p 4 m m$, shown in Figure 3.10, in which case we would expect to see two sets of two perpendicular mirror lines, oriented at 45 degrees, as well as two rotation centers around which a 90 degree rotation is a symmetric operation (known as a type 4 rotation center), or a site symmetry of $4 \mathrm{~mm}$. A cursory examination of the raw image shows no such mirror lines, nor are there conspicuous rotation centers.
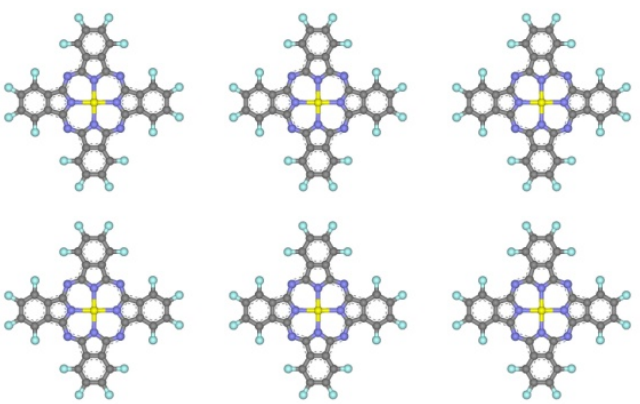

Figure 3.10. Drawing of $\mathrm{F}_{16} \mathrm{CoPc}$ molecules arranged with $p 4 m m$ symmetry; note this is a possible alignment, not necessarily observed.

The raw image is 1771 pixels on each side. The implementation of the discrete Fourier transform (DFT) in the software used in this section (CRISP [26]) samples either square areas of an image with a side equal to a power of 2 , or circular areas with a diameter equal to a power of 2 . We have found that the square sample results in Fourier transform plots with rectangular artifacts aligned with the sides of the sample due to 
edge effects, and that circular samples have "cleaner" Fourier transforms and provide adequate results. The software also allows the user to select one irregular area in the image to be "grayed out"; set to the average pixel level of the rest of the image.

The Fourier transform of an aperiodic function is itself aperiodic. But when the transform is applied to a periodic function, the output is discrete. In the particular case of a two-dimensional periodic array, if the periodicity is mathematically perfect and infinite), the transform $f(x, y) \rightarrow \mathrm{F}(H, K)$ results in an array of mathematical points. A real periodic image of course departs from perfection but the Fourier transform output will largely be concentrated in "spots".

Figure 3.11 shows the amplitudes of the two-dimensional DFT as applied to three separate 1024-pixel diameter samples taken in different parts of the image; one at the top center and two in the lower left and right corners respectively. Figure 3.7 appears uniform across the array. The DFT images below confirm that; there is no obvious difference in spot location or intensity between the three areas, and CIP using any of the
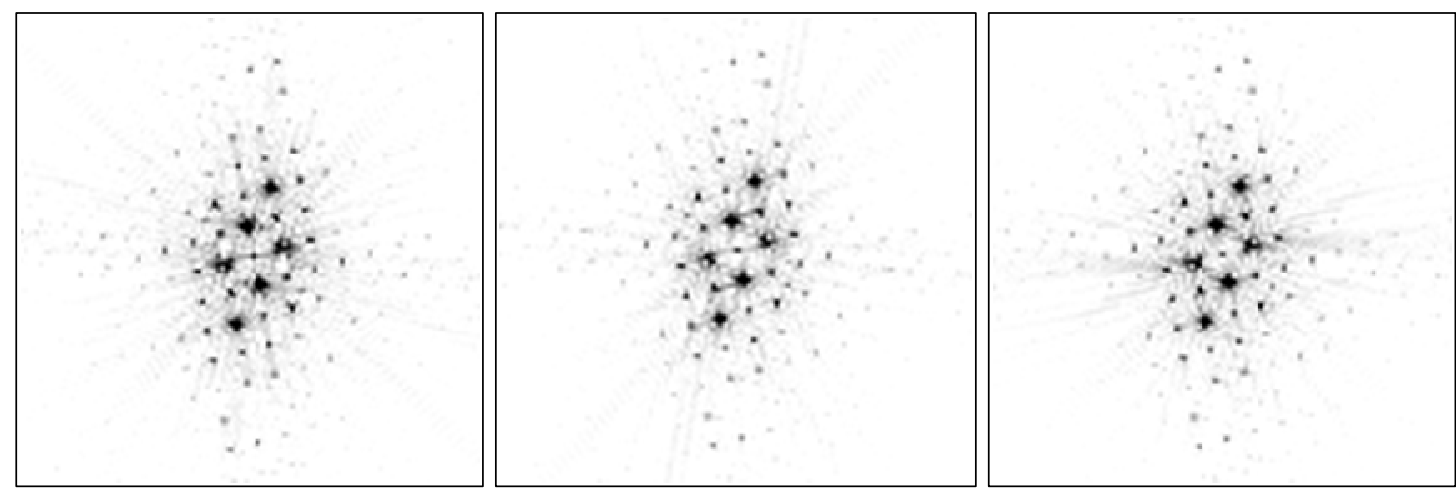

Figure 3.11. Amplitude portion of DFT of the raw image in figure 3.7, taken from three separate areas in the image. Note similarity between the DFTs, implying that the image is very uniform. 
three gives virtually identical results (see Table 3.1). Also note the number of spots visible in all of the Fourier transforms and compare with figure 3.8, the Fourier transform of pure HOPG. This confirms that Figure 3.7 is more than just graphite.

\begin{tabular}{|l|l|l|l|}
\hline \multicolumn{3}{|c|}{$\begin{array}{l}\text { Table 3.1. Dimensions of the reciprocal lattice selected for each of the three sample areas; note they } \\
\text { are almost identical, showing that the Fourier amplitudes of the sample are translation invariant. }\end{array}$} \\
\hline & Sample area 1 & Sample area 2 & Sample area 3 \\
\hline$a^{*}$ (arbitrary units) & 7.4 & 7.5 & 7.5 \\
\hline$b^{*}$ (arbitrary units) & 7.8 & 7.8 & 7.8 \\
\hline$\gamma^{*}$ (degrees) & 84.0 & 84.4 & 84.3 \\
\hline
\end{tabular}

\subsection{Processing of the image}

These transforms (and the others in this chapter) were calculated using the program CRISP. The images of the Fourier transform were black/white reversed in order to show the detail more accurately. The output extends in theory to infinity in both the $x^{*}$ and $y^{*}$ directions but the long range periodic information is concentrated near the origin of the reciprocal lattice.

The next step in CIP is to take the Fourier transform (one of the three above was chosen arbitrarily) and ascertain the dimensions of the reciprocal lattice. The calculation of the transform is done automatically. The selection of the axes also can be done automatically in the CRISP program, subject to override by the user, who may manually index the observed lattice.

Before proceeding with axis selection (either automatically by software or manually) the user may choose to manually filter out higher-order Fourier coefficients, which correspond to shorter range periodic information. This is done by restricting the 
analysis to points in the interior of a circle around the reciprocal space origin. To do this is to make a subjective judgment as to the validity of higher-order coefficients, but one can make a semi-quantitative justification for the filtering by considering the smallest feature likely to be visible in the molecule.

For this section we chose a circle with a radius of 100 pixels. Figure 3.12 shows reciprocal axes overlaid on a Fourier transform.

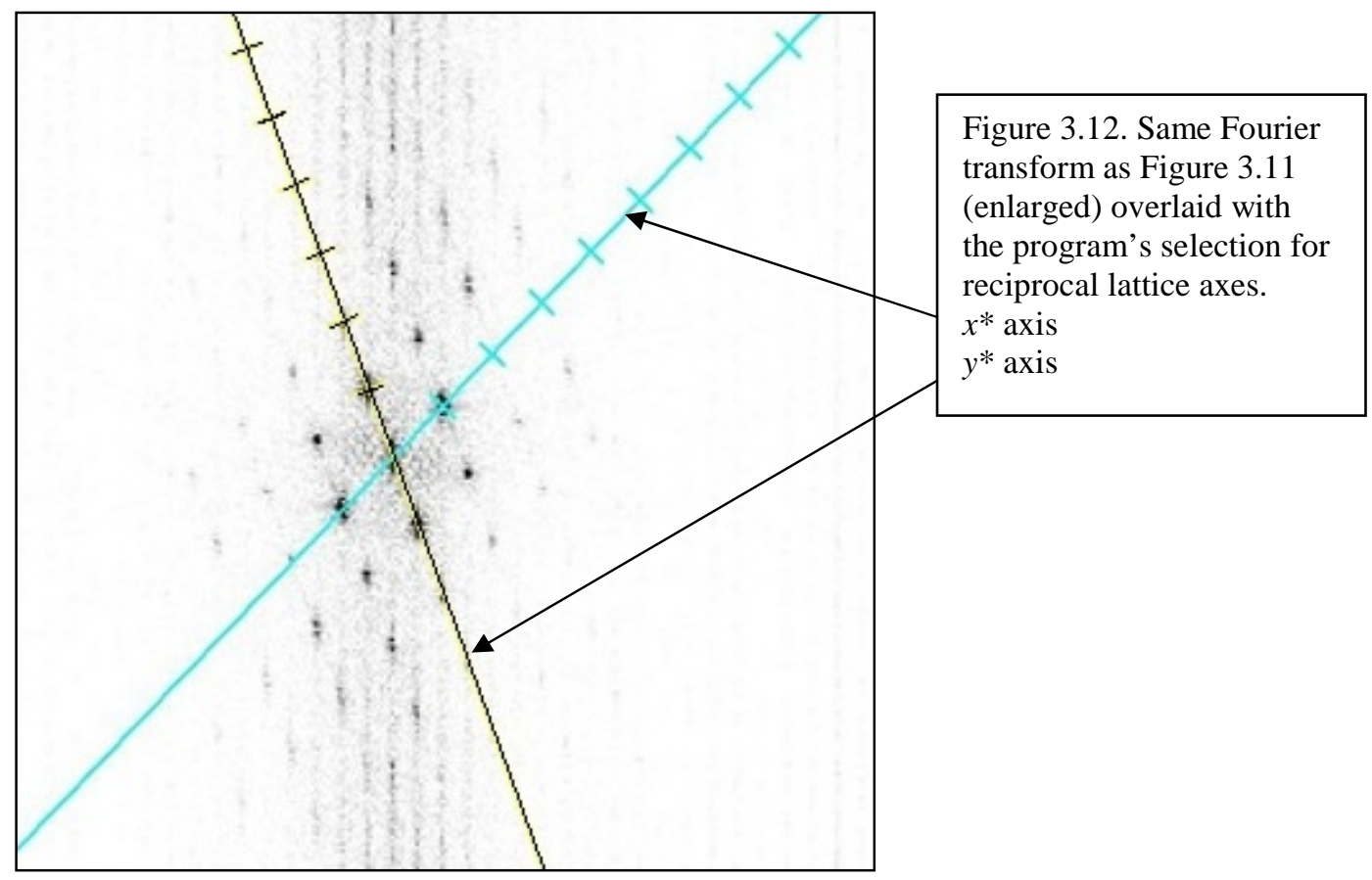

The blue and yellow axes represent the $x^{*}$ and $y^{*}$ reciprocal axes respectively that have been proposed by the program. The user is expected to visually compare the alignment and scale of the proposed axes with the displayed spots, using the unique discernment of the human eye in order to verify that the software is finding the correct lattice and has not been misled by noise. 
The decision to restrict the analysis to a circle around the origin of reciprocal space is essentially a decision to filter out higher-order Fourier coefficients, since those by definition are the coefficients that are distant from the origin. In the particular case of this image, we have tested both the 100 pixel circle used and a 512 pixel circle that covers the full reciprocal image and have found little or no difference in the results. In both cases we obtain 58 Fourier coefficients for $p 4 m m$ symmetry (subject in both cases to the same dynamic range amplitude cutoff) and the residuals are almost identical: 28.1 for the RA\% of both the 100 and 512 pixel selection, and a phase residual of 34.2 for the 100-pixel circle and 34.7 for the 512-pixel circle. However for other images the decision to filter out higher-order coefficients may assist in removing aperiodic information and noise. We do not believe there is a perfect solution other than to anticipate the smallest feature likely to be observed in the sample (perhaps a bond length) and disregard any periodic feature in the image smaller than that.

The heart of the program and essence of CIP is the process that follows. One wants to ascertain which of the 17 plane groups the raw image best matches. Although the human eye can again make a rough estimate, by taking advantage of computational power one can simply test all of the groups, considering all possible origins throughout a unit cell for all groups.

The output of CRISP and other CIP programs actually differentiates between 21 settings of the plane groups rather than the 17 plane groups in their standard setting. This is because for groups $\mathrm{pm}, \mathrm{cm}$, and pmg that incorporate a single mirror line, and for 
group $p g$ that incorporates a single glide line, the same group can be viewed as with either the $\mathrm{x}$-axis or the $\mathrm{y}$-axis parallel to the single line. The selection of axis orientation changes the residuals for various Fourier coefficients, and thus output is displayed for the two different orientations, but each of these different settings is still only one plane group.

The groups $p 3, p 3 m 1, p 31 m, p 6$, and $p 6 m m$ by definition have lattices with axes inclined at 120 degrees in direct space. The translational symmetry along those axes supports symmetry elements 3 or 6 at the origin. The axes in reciprocal space have a 60 degree angle. The remaining groups (with the exception of the $p 1$ and $p 2$ groups) have an overall rectangular appearance with 90 degree angles between the axes, this time in both real and reciprocal space. CRISP and other CIP programs make a first decision about which subset of potential plane groups to follow based upon the overall alignment of the reciprocal axes. In this case, the angle of 95.9 degrees in direct space is close enough to 90 degrees that 15 groups with an overall rectangular orientation are the ones chosen for further calculation.

It is important to note that if the angle between the axes is not close to either 90 or 60 degrees, the only logical plane groups are $p 1$ or $p 2$; that is to say, for $p 1$ no symmetry exists at all other than translational symmetry, the simple two-dimensional periodic repetition of a pattern, while for $p 2$ there exists a set of 2 fold rotations.

In order to identify an origin, CIP software in general starts from a random point and tests every pixel of the unit cell in real space to find the point (and its translation 
symmetric equivalents) about which the symmetry is best (or least broken). For certain symmetry groups, there is no one point but there are lines of symmetry along which the origin can be located. Once an origin is found, residuals are calculated for that plane group as described earlier in this thesis.

Once the residuals have been calculated for each of the possible plane groups (again this is an objective procedure), the somewhat subjective decision of which plane group fits the data best can begin.

Plane groups are not equal in the sense of "quantity of symmetry" that they contain. This is best seen by considering the "multiplicity" that in the case of a primitive unit cell is the number of times that the "general position" (any location not lying on a symmetry element) is replicated in the unit cell as a result of the symmetry operations associated with that group. In other words, if one takes an arbitrary point in the unit cell it is the number of copies of that point that can be found elsewhere in the cell. With the exception of $p 1$, each group also contains "special positions", the term for a location on a symmetry element, e.g. on a rotation axis, on a mirror line, etc. Special positions have a lower multiplicity than the general position since they are not transformed by the element on which they reside, but there are only a finite number of special positions whereas there are an infinite number of general positions in any unit cell. In the case of a $2 \mathrm{D}$ image, the number of general positions is finite (since the number of pixels is finite) but still much greater than the number of special positions. This justifies the conclusion that general position multiplicity is a good measure of quantity of symmetry. 
For a centered plane group $(\mathrm{cm}$ or $\mathrm{c} 2 \mathrm{~mm})$ the number of copies of the general position that can be obtained for the primitive cell counterpart $p m$ or $p 2 m m$, must be multiplied by 2 , the number of primitive cells in the unit cell. The result is the multiplicity.

In general, between two plane groups with similar residuals, the plane group that contains general positions of higher multiplicity would be preferred over one with lesser multiplicity. The reasoning is that a group with a high multiplicity general position (such as $p 4 m m$ with its multiplicity of 8 ) has eight different positions in the cell that, when symmetry is enforced, will have their intensities averaged, as opposed to a low multiplicity group (such as $p 2$, which has a multiplicity of 2).

One should also keep in mind the point symmetry of the underlying molecule and attempt some reasonable accommodation to the likely arrangement of that molecule in an array. In the case of the transition metal phthalocyanine seen here, $p 4 m m$ would be a likely arrangement if the molecule is not distorted by interaction with the substrate (a substrate that has, in the case of HOPG, $p 3 m 1$ symmetry).

Figure 3.13 shows the output from the CRISP program after residual calculations. On the left is a list of the 21 settings of the 17 plane groups (in the notation here, the pm, pg, cm, and pmg groups are each treated as two separate settings depending upon whether the mirror/glide line is oriented in the $x$ or $y$ direction) the residuals for that group, where applicable, and the Ao/Ae figure of merit (described below). (Another notation difference in CRISP is the abbreviated notation for what are known in the 
International Tables for Crystallography [4] as p2mm, p2mg, p2gg, c2mm, p4mm, p4gm and $p 6 m m$ groups as pmm, pmg, pgg, cmm, $p 4 m, p 4 g$, and $p 6 m$.

Note that the hexagonal groups are not considered; their residuals are blank. The column labeled $R A \%$ is the amplitude residual. The column labeled $\varphi \operatorname{Res}$ is the phase residual. The column labeled $A o / A e$ is a different figure of merit for the plane group; it is meaningful only for the six groups that have "systematic absences", that is certain Fourier coefficients for which the innate geometry of the group requires that reflections from the symmetry-related locations in the unit cell cancel out, in other words have zero amplitude. In reality experimental error and thermal agitation prevent these amplitudes from actually going to zero. Ao/Ae is the observed amplitude of these expected zero reflections divided by the sum of the amplitude of the other, permitted reflections. It will be zero in a perfect sample.

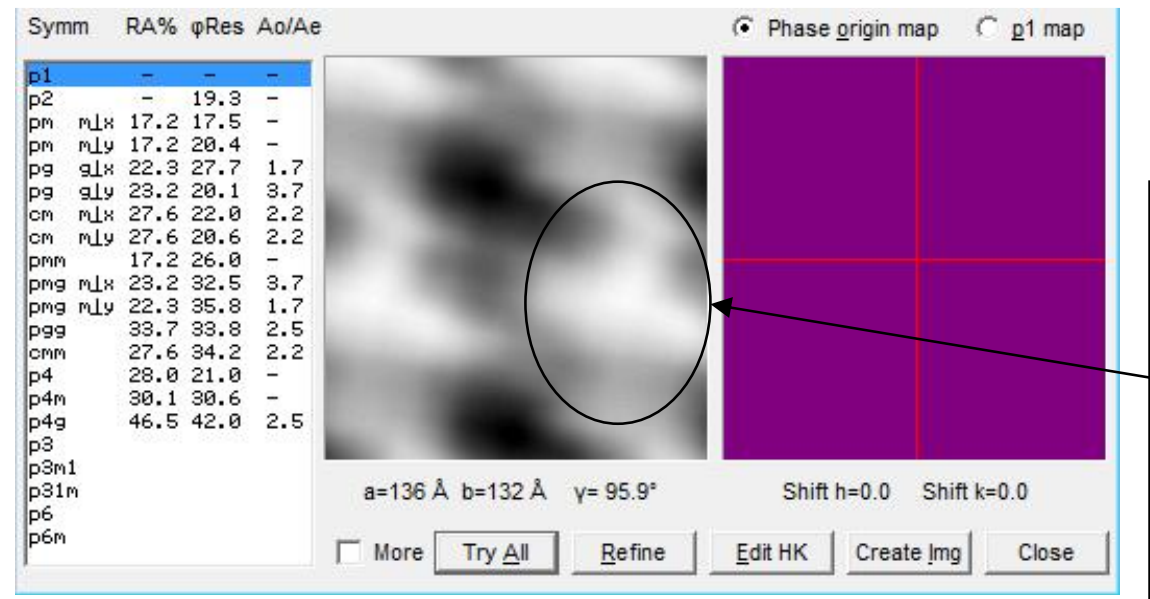

Figure 3.13. The left portion of the image is a list of possible plane groups and, where applicable, their residuals; in the center a reconstruction of the image enforcing whatever symmetry is highlighted in blue on the left ( $p l$ in this case).
This is a real space reconstruction applying $p 1$; circled, one can see the molecule beginning to take shape as successively higher plane symmetries are enforced. 
Note that when the Ao/Ae ratio is over 1.0 for each of the applicable groups, it makes it relatively unlikely that these are the proper symmetry for this sample. This leaves $p 1, p 2$, the two $p m$ groups, $p m m$, $p 4$, and $p 4 m m$ as viable candidates.

The $p l$ group is an interesting case because it is the group that asserts there is no point symmetry in the unit cell and no overall symmetry other than translational; in other words a two-dimensional array with only $p 1$ symmetry is not symmetric other than the ability to translate. This also means that enforcing $p l$ symmetry is equivalent to the well-known technique of translational averaging. Such enforcement effectively sums the real-space amplitudes of all the unit cells together and divides by the number of unit cells, averaging noise and other random fluctuations across all cells while enhancing the truly translationally periodic elements of the image.

The noise suppression advantage of CIP is due to the additional averaging that takes place at the various symmetry elements, and in fact is more pronounced as the plane group is of higher symmetry. The demonstration of this is made clear by observing this $p 1$ reconstruction (shown in the center of figure 3.13) which expresses how the image can be averaged without any additional contribution from CIP. Notice that the appearance of the reconstruction is not greatly different from the raw image unit cell; the irregularities of shape are preserved (as they must be in group $p 1$ ).

The enforcement of plane group $p 2$ is shown in figure 3.14 and has a calculated phase residual of 19.3 degrees, which is the only meaningful residual for $p 2$ since for this group there are no amplitude symmetry relations between the various Fourier 
coefficients, other than the Friedel pair relationship $F(h, k)=F(-h,-k)$ which is a mathematical consequence of the Fourier transform. This is considered a low residual in electron crystallography, and in fact proves that the raw image has a degree of $p 2$ symmetry, i.e. there exist points about which the image can be rotated 180 degrees with little change (if the $p 2$ symmetry were perfect, there would be no change). The enforcement of that symmetry results in the image shown in the center of figure 3.14 , and one can begin to see in the circled cross (one of two that appears in the image) the outline of what could represent a cross-shaped molecule with $4 \mathrm{~mm}$ symmetry. Note that the effect of this enforcement is simply to equate the number of pixels on either side (180 degrees apart) of a 2 -fold rotation axis.

Figure 3.14 also shows the "phase map", which is the multi-colored panel on the right side of the figure. This is a view of approximately two unit cells in real space and is a plot in which the $x, y$ position represents a tentative location for the origin, and the color corresponds to the phase residual if the original were at that location. Since the goal of CIP is to minimize residuals, a valley in this map represents a good origin. The contours of the phase map help the user see how sensitive the residuals are to original location. 

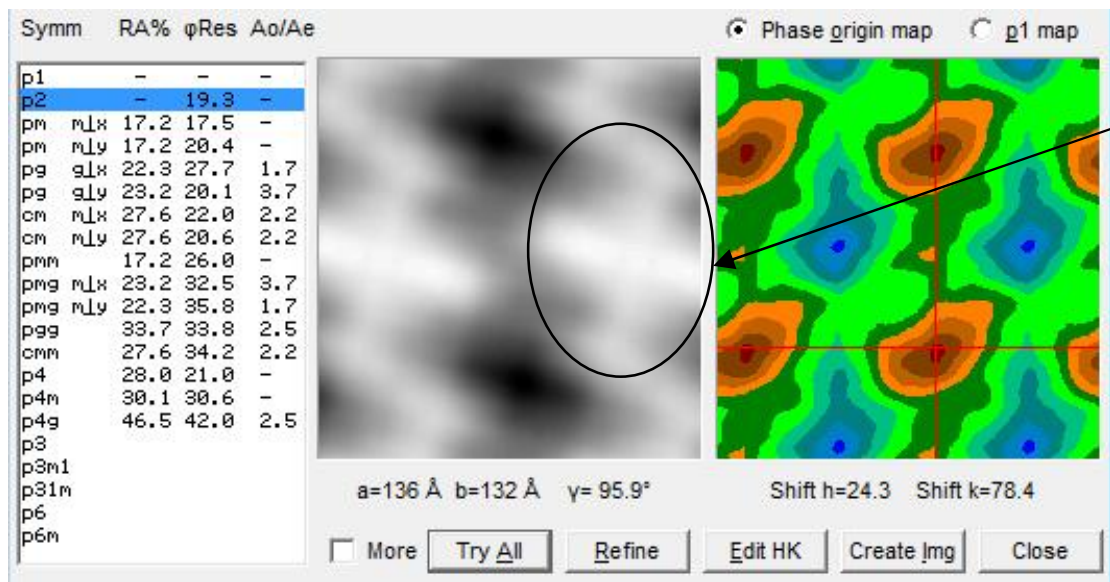

$p 2$ enforces site symmetry 2 on the periodic motif

Figure 3.14 Output from CRISP, same area of sample as Fig. 3.13 but with $p 2$ group chosen for enforcement.

Although the low residual for $p 2$ and the visibly logical reconstruction is encouraging, the remaining plane groups have many more symmetry elements than $p 2$ so it is helpful to move on to them.

We see relatively low residuals for the two $\mathrm{pm}$ groups and the $\mathrm{pmm}$ group. But their multiplicity is low compared to the $p 4$ and $p 4 m m$ that do in fact have higher residuals. The highest multiplicity within this selection of groups, 8 , is a property of the $p 4 m m$ group. Because of the fact that $p 4 m m$ contains the mirror planes of the $p m$ groups plus a 4-fold rotation point, the general position in the unit cell is copied seven times throughout the cell; thus eight copies of each general position exist. Special positions (which lie on a symmetry element) have lesser multiplicity.

Figure 3.15 shows the results of enforcing $p 4$ symmetry, which equates the pixels along any pair of axes 90 degrees apart from a 4-fold rotation point, but without enforcing any mirror lines. The result is beginning to look more like the phthalocyanine molecule, although without the bright center characteristic of cobalt phthalocyanine 
[27]. The molecule in this image also has subtle differences on opposite sides of where one would expect two of the mirror lines to be, along the longitudinal axes of the extended "arms" of the molecule. Since the molecule itself has $4 \mathrm{~mm}$ point symmetry we assume a tip asymmetry causes the lack of mirror symmetry.

Figure 3.16 shows several results when $\mathrm{p} 4 \mathrm{~mm}$ symmetry is enforced, the highest symmetry that this layer of molecules would have if the point symmetry of the isolated molecule, $4 \mathrm{~mm}$, were to be the site symmetry in the array. Notice immediately the bright spot at one 4-fold rotation point and a vacancy at the other 4-fold rotation point. This bright spot is in agreement with Hipps' work that shows STM images of cobalt phthalocyanines have high tunneling current at the Co atom, which thus appears as a bright spot. It is encouraging that enforcing $p 4 m m$ symmetry (that contains the known $4 m m$ symmetry of the solo molecule) results in an image consistent with what one could expect from a good STM image.

Figure 3.16(d) collects and displays the periodic motif of each of the four different plane groups enforced in this section. 


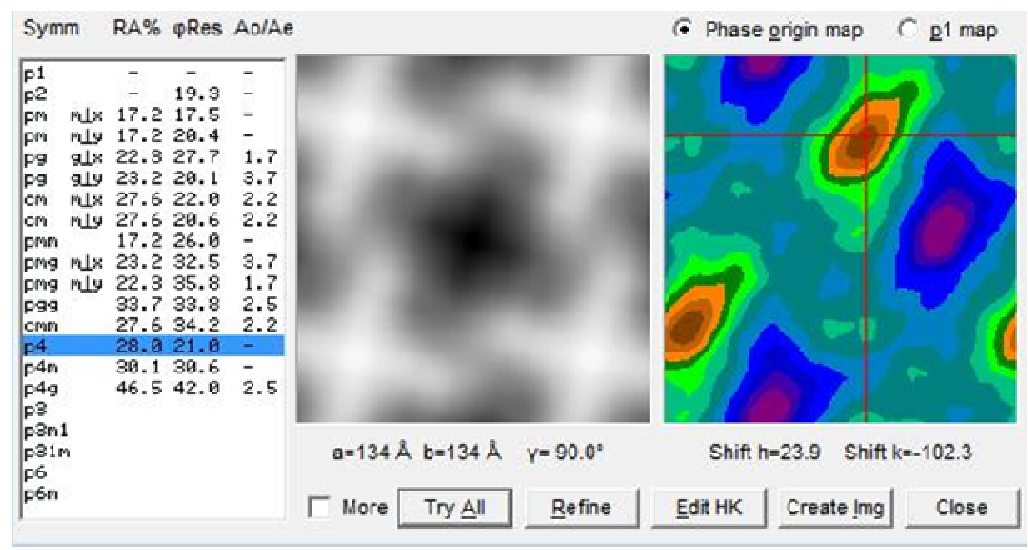

Figure 3.15.

Same area as

figure $3.13, p 4$

enforced. The periodic motif now has site symmetry 4.

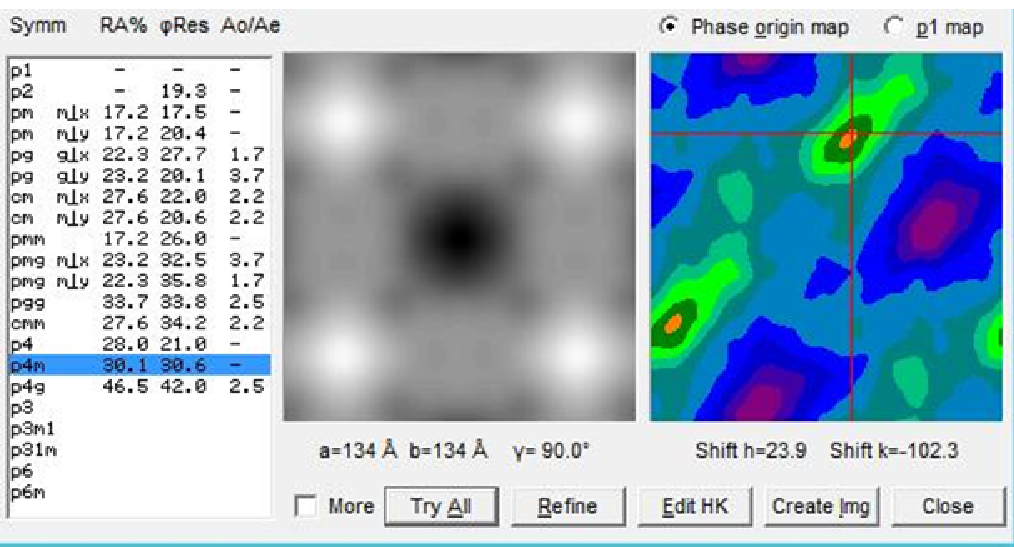

Figure 3.16a.

Output from same area as figure 3.13 with $p 4 m m$ enforced. The periodic motif now has site symmetry $4 m m$.

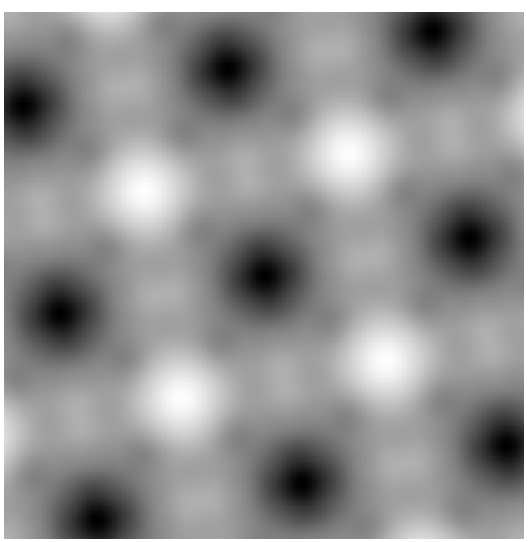

Figure 3.16b. Approximately four unit cells of $p 4 \mathrm{~mm}$ enforced, axes inclined as in raw image. 


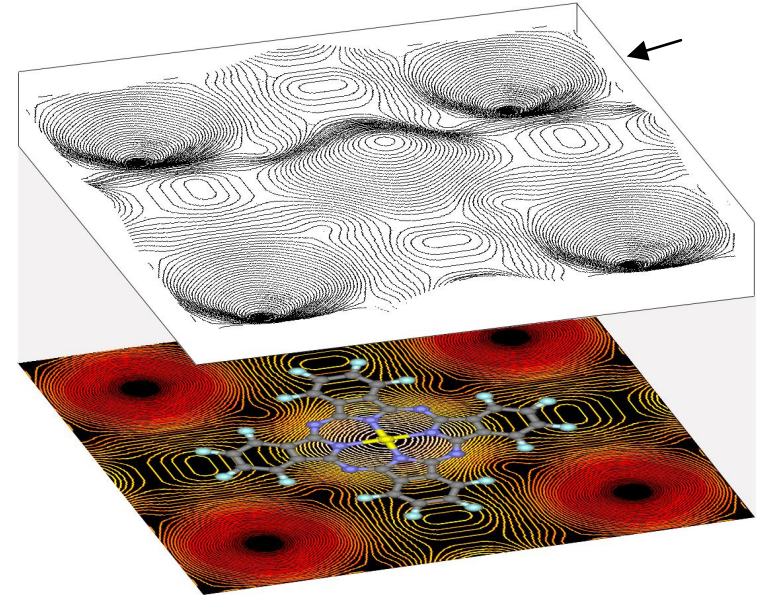

Figure 3.16c. Contour plots of 1.5 unit cells of the $p 4 \mathrm{~mm}$ data with one molecule sketched in. The contour plot converts the intensity distribution of Fig. 3.16(b) into 64 levels, while the $3 \mathrm{D}$ presentation clearly shows the peaks and valleys. (from [23])

Figure 3.16d. Contour plots, 64 levels, of the image after the four plane groups mentioned in this section enforced; approximately one periodic motif shown; clockwise from top left: $p 1, p 2$,
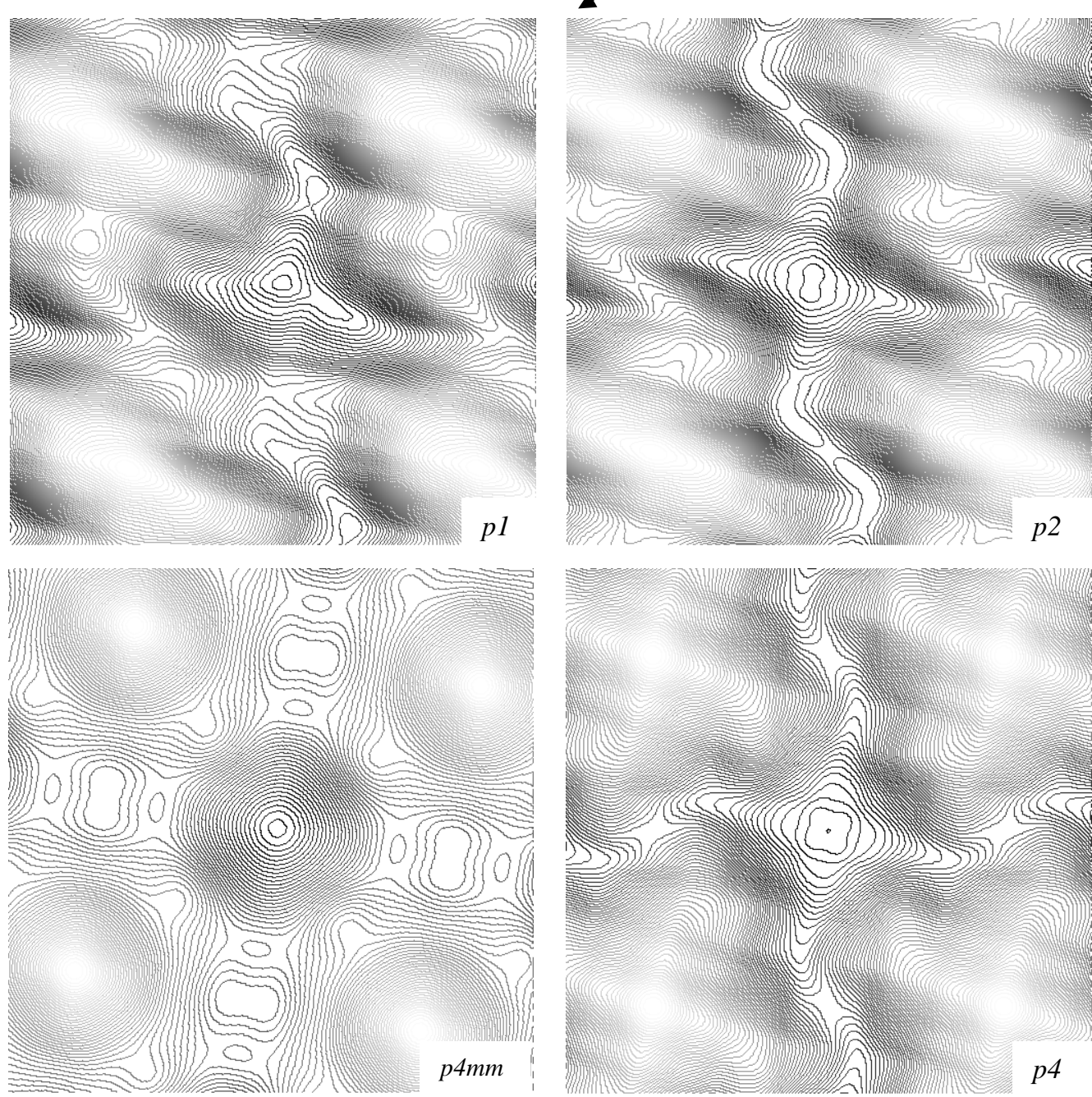


\subsection{Translational averaging compared to CIP}

Figure 3.17 shows a simulated image of $p 4 m m$ symmetry. The motif, a cross, has $4 m m$ point symmetry like the organic molecule discussed earlier. Figure 3.18 is the same image with the motif changed in each periodic location by the movement of a pseudorandom unit-sized chunk. There are the same number of dark pixels in each image.

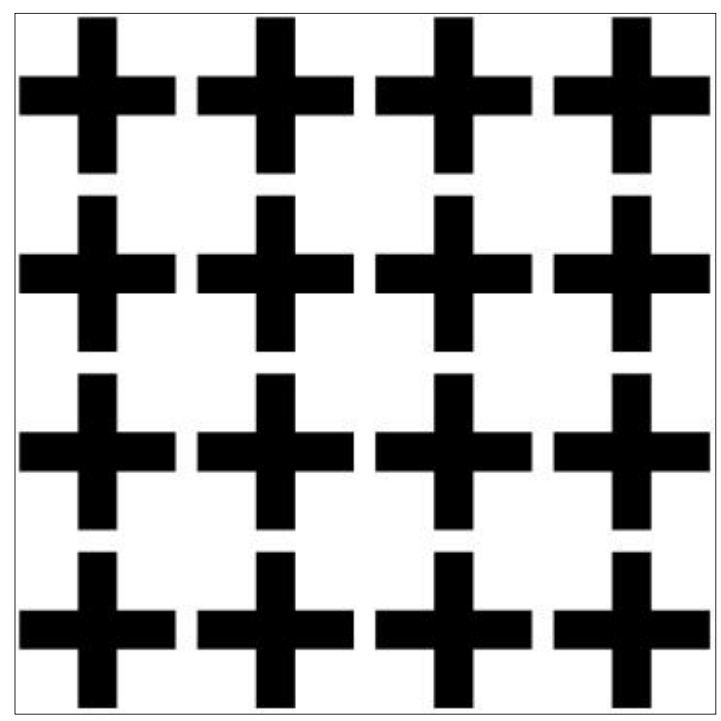

Figure 3.17 A p $4 m m 360 \times 360$ pixel artificially generated image.

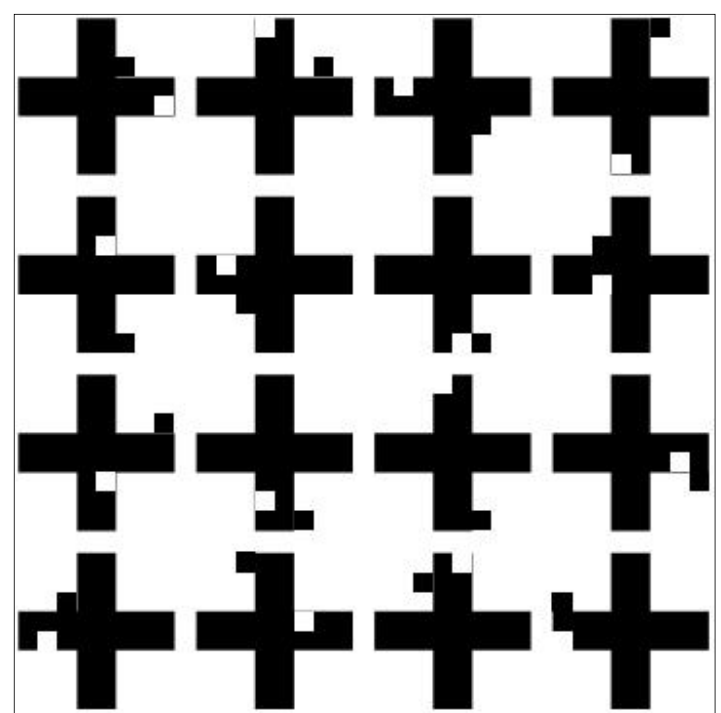

Figure 3.18. A $360 \times 360$ image that has been pseudorandomly distorted.

If one assumes that the undistorted image represents the "true" arrangement of the sample, and the right image is an observation that has been distorted by noise or random experimental error, it is interesting to process the distorted image and see how translational averaging and CIP perform. 
Translational averaging is the equivalent of enforcing $p l$ symmetry; once the lattice dimensions are determined, the individual unit cells are effectively laid on top of one another and averaged. Applying this to the right side of figure 3.18, the distorted image, results in figure 3.19. Figure 3.20 a closeup of the periodic motif.

If one considers that the desired outcome is the "true" motif that is a pure blackon-white cross, translational averaging does not do a bad job. The jagged edges of figure 3.18 are gone. However the prominent bright splotch in the lower left hand corner of the unit cell as well as the irregular shading of the cross is evidence that the pseudorandom distortions are not averaging into insignificance.

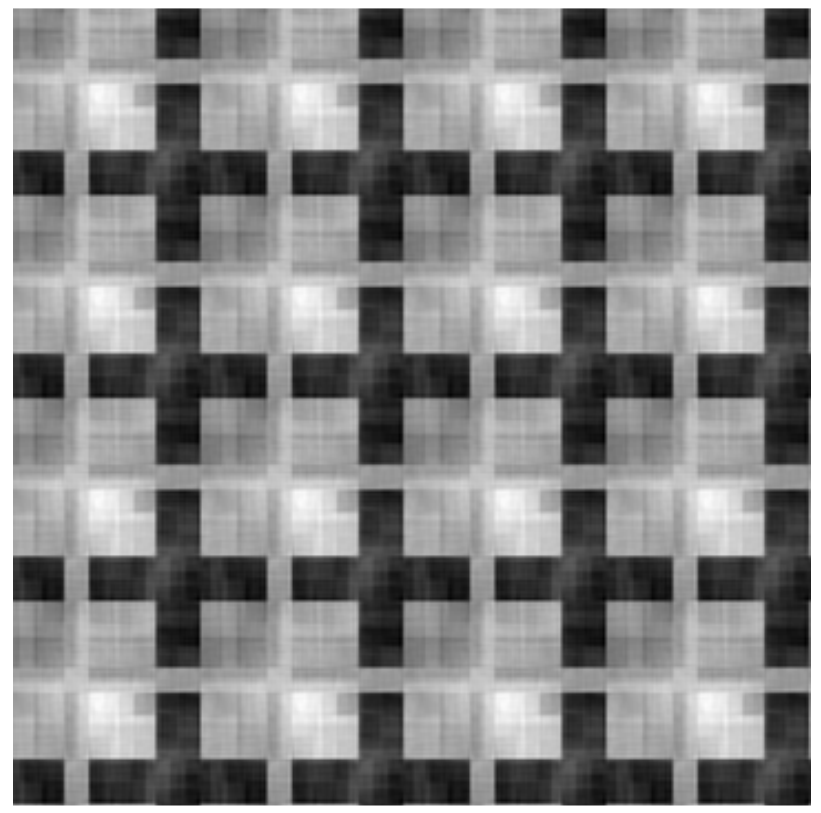

Figure 3.19. The result of $p 1$ enforcement (translational averaging) on the distorted figure 3.18 .

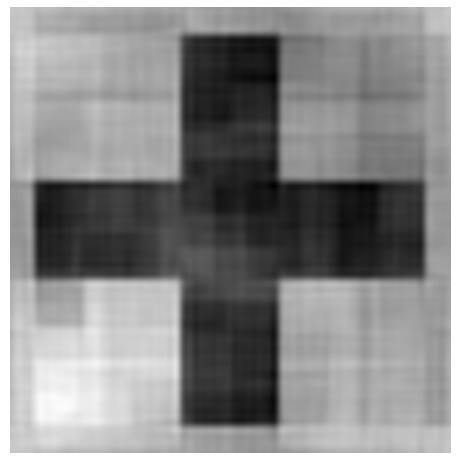

Figure 3.20. Closeup of $p 1$ enforced unit cell. 
Consider figure 3.21 (and the closeup of figure 3.22), the result of $p 4 \mathrm{~mm}$ enforcement. The enforcement of the rotational and mirror symmetry of the plane group (taking advantage of our prior knowledge of the cross motif) results in an image much closer to the cross motif as it looked before distortion. The irregularities in the cross as well as the bright splotch are gone. Note that the averaging is only over 16 unit cells. The artifacts that remain are because of the necessity of selecting a finite area of real space with which to calculate the Fourier transform.

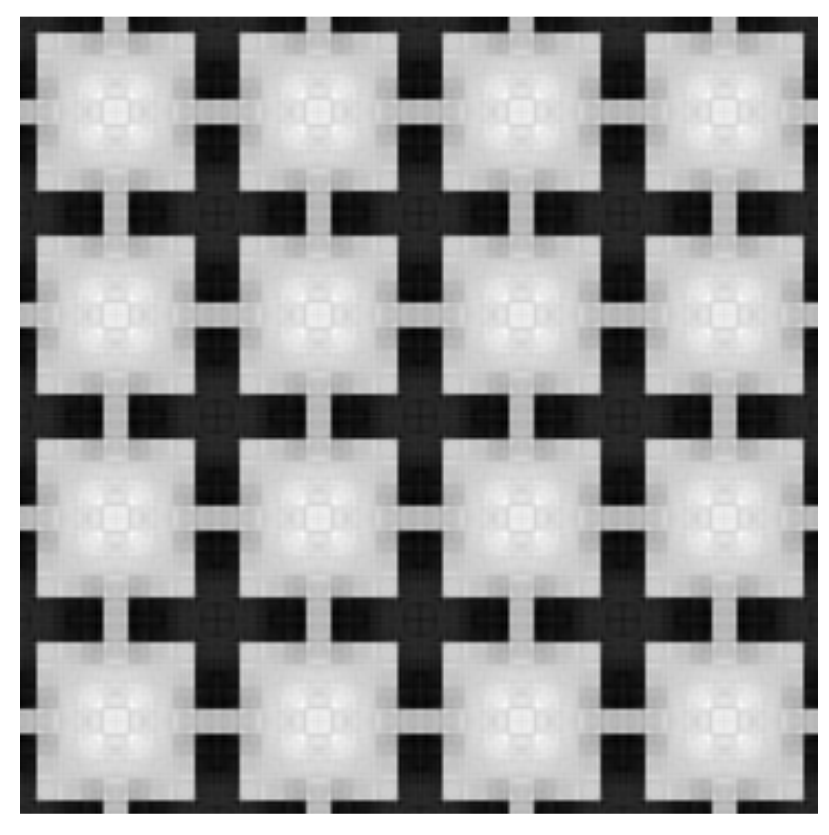

Figure 3.21. After enforcement of p4mm symmetry.

Figure 3.22. Closeup of $p 4 m m$ enforced unit cell. 


\subsubsection{Effectiveness of CIP compared to translational averaging}

When the dispersion of "errors" is truly random (or as random as the pseudorandom number generators of Mathematica and Visual Basic allow) the relative advantage of CIP as compared to simple translational averaging becomes less apparent the larger the number of unit cells. This is to be expected as we are essentially observing the standard error of the location of the irregularity, and as sample size (number of unit cells) increases the standard error decreases:

$$
S E=\frac{\sigma}{\sqrt{n}}
$$

where $\sigma$ is the standard deviation of the distribution and $n$ is sample size.

For a simple example consider repeating the above experiment with a $6 \times 6$ matrix of motifs instead of $4 \times 4$ :

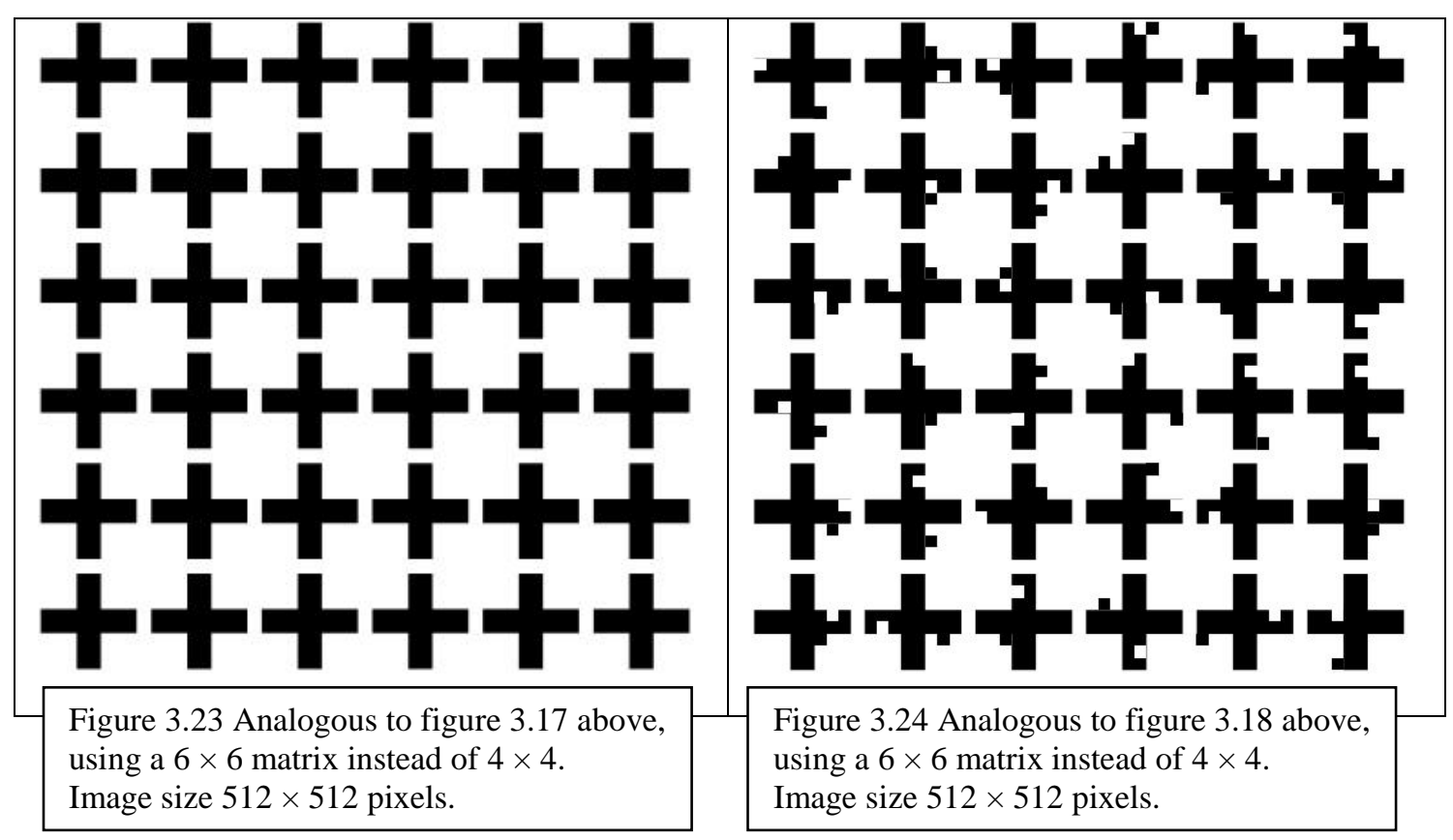


The same pseudorandom technique from Mathematica is used for the placement of the irregularities in Figure 3.24. But the fact that there are 36 cells instead of 16 creates the visual impression that $p l$ averaging is much more successful. See Figures 3.25 and 3.26 for the $p 1$ results. Figures 3.27 and 3.28 show analogous results for $p 4 m m$ enforcement.

Although the translational averaging output is certainly closer to the "true" image in this case, examination of figure 3.26 shows subtle asymmetries that are not part of the undistorted image. In particular, the black body of the cross is far from uniform across its extent. This is not the case for figure 3.28, the $p 4 \mathrm{~mm}$ enforced cell. Thus it is still fair to state that CIP has enhanced the value of the image over and above translational averaging.

The margin of superiority of $p 4 \mathrm{~mm}$ enforcement over $\mathrm{pl}$ enforcement grows smaller with increasing sample size, until with a large enough sample they might be visually indistinguishable. However $p l$ enforcement is unable to remedy systematic error. CIP, on the other hand, can correct for systematic error given some prior knowledge of the sample, as seen in Figures 3.29 through 3.35. These figures greatly increase the sample size and introduce some deliberate systematic error. 


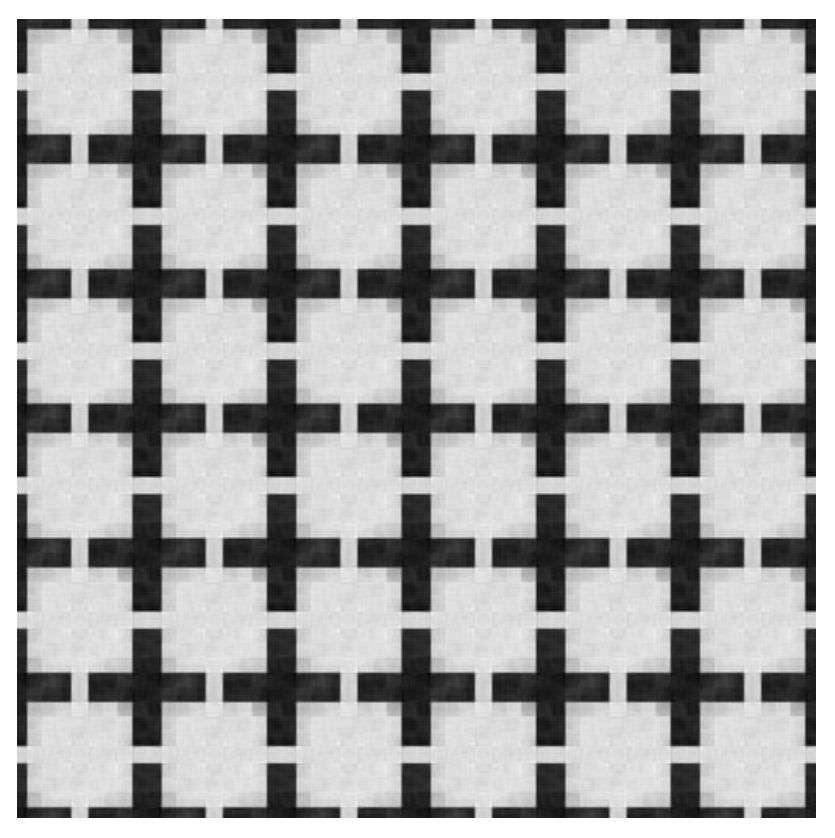

Figure 3.25. The result of $p l$ enforcement on the distorted figure 3.24 .

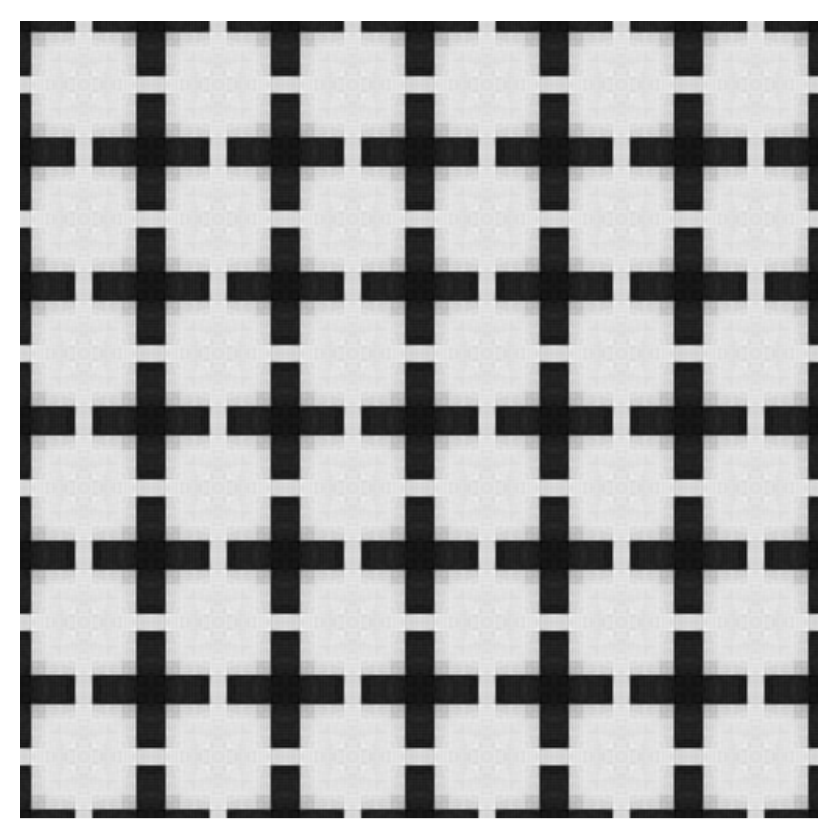

Figure 3.27. The result of $p 4 \mathrm{~mm}$ enforcement on figure 3.24.

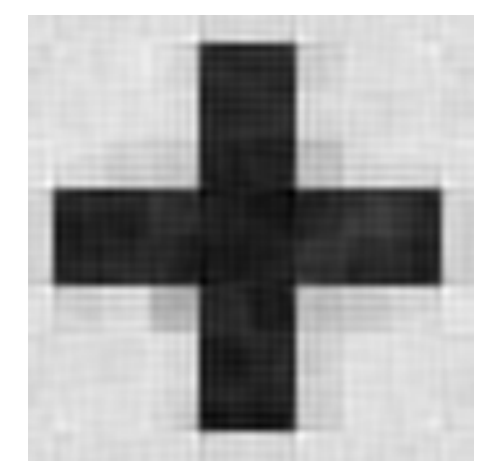

Figure 3.26. Closeup of $p 1$ enforced unit cell. There is subtle asymmetry but this is close to the "true" motif.

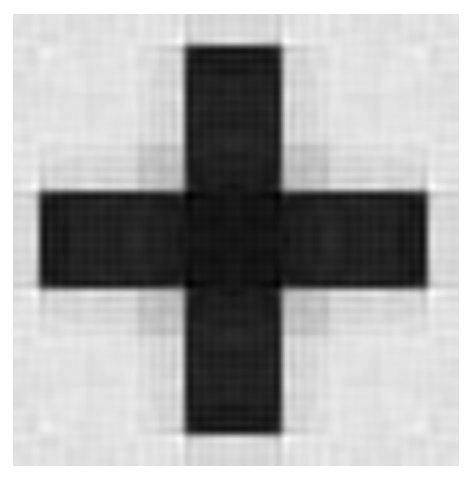

Figure 3.28. Closeup of $p 4 \mathrm{~mm}$ enforced unit cell. This is closer still. 
We now greatly increase the size of the array and use the same analysis. If all error is random, the result using CIP is virtually indistinguishable from the result of translational averaging. See figure 3.29 which shows an array of 50 rows and 50 columns of crosses, while figure 3.30 is the same image with random distortions. (For

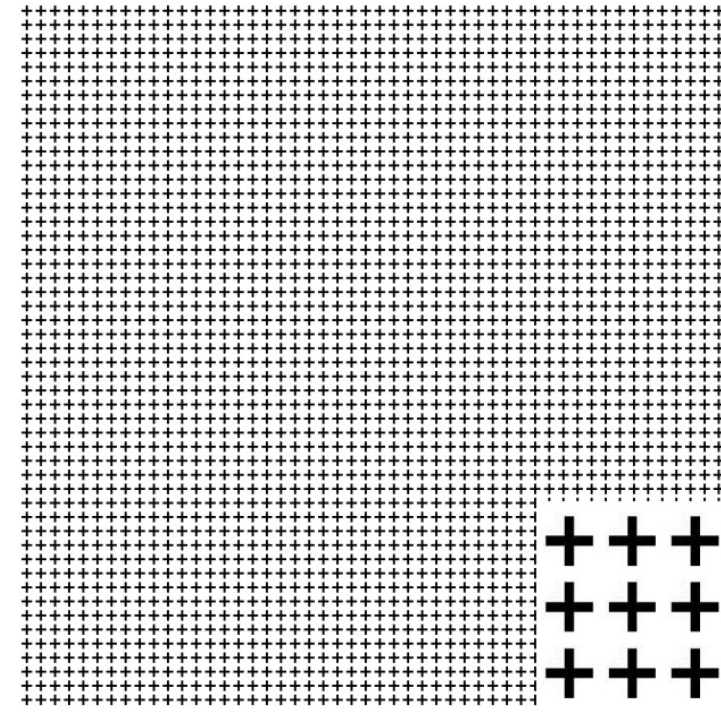

Figure 3.29. $2048 \times 2048$ pixel image containing $50 \times 50$ crosses.

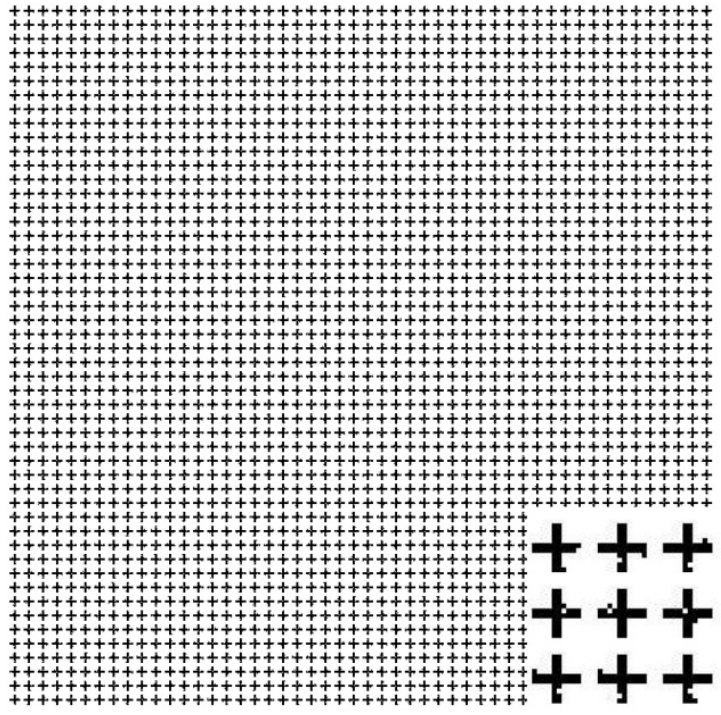

Figure 3.30. Similar image containing crosses with one block of pixels displaced.

both images the inset is to show the detail of the array, and is not processed by CIP).

When we apply CIP to the image with random errors, and enforce first translational symmetry $(p 1)$ and then $p 4 m m$ symmetry, the two corrected images in 3.31 are virtually identical regardless of whether $p 1$ or $p 4 \mathrm{~mm}$ symmetry is enforced.

This demonstrates that a sufficiently large number of random errors in an image can be efficiently averaged out by translational averaging. The similarity between the pl results and the $p 4 m m$ results is consistent with equation 3.1. Consider that the standard error of the location of the irregularity varies as $1 / n^{1 / 2}$ where $n$ is the sample 
size. Enforcing a higher symmetric plane group means the standard error now varies as $1 /(n \cdot \text { multiplicity })^{1 / 2}$. As $n$ gets larger, $\left|\sigma / n^{1 / 2}-\sigma /(8 n)^{1 / 2}\right|$, which is the difference between the $p 1$ standard error and the $p 4 m m$ standard error, shrinks, with a limit of zero as $\mathrm{n}$ goes to infinity.

However, systematic errors such as those caused by less than perfectly calibrated piezoelectrics that are not acting exactly proportional to each other cannot be remedied through translational averaging. CIP can deal with systematic error and is clearly advantageous if such error is present.
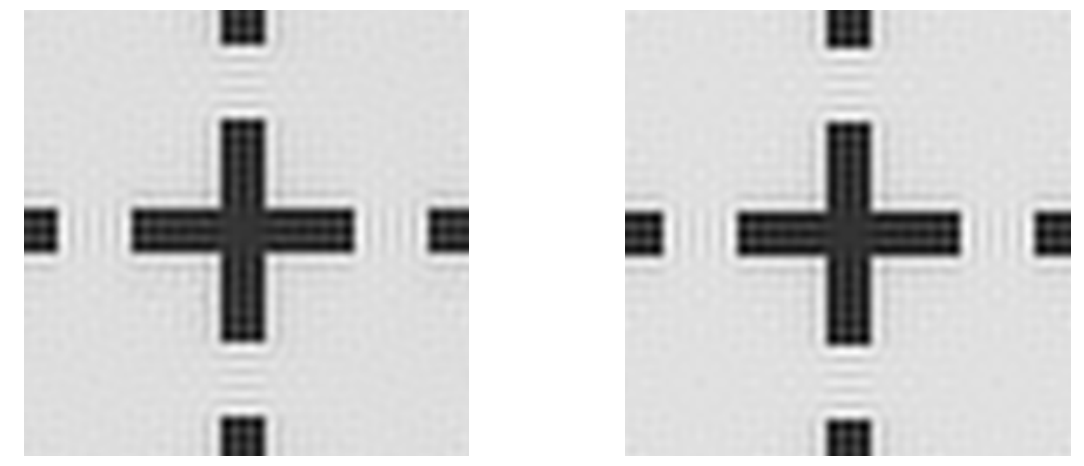

Figure 3.31. Closeups of periodic motifs of CIP processed distorted image in Fig. 3.30. (left) $p 1$ enforced (right) $p 4 m m$ enforced. Note virtually no visible difference.

Figure 3.32 shows another distorted image of 50 rows and 50 columns of crosses, but with a different type of distortion; a unit-sized chunk has been removed from a random location on each cross but replaced only on the upper arm of the cross. This introduces a bias into the array of crosses that is intended to simulate systematic error. 
When this image is processed using CIP and enforcement of $p 1$ and $p 4 m m$ symmetries are compared, as we see in Figure 3.33 there is a subtle but clear difference in the quality of the resulting image. Enforcing the high symmetry of $p 4 m m$ removes the asymmetry that is visible in the $p l$ image. We conclude CIP is valuable in removing systematic errors.

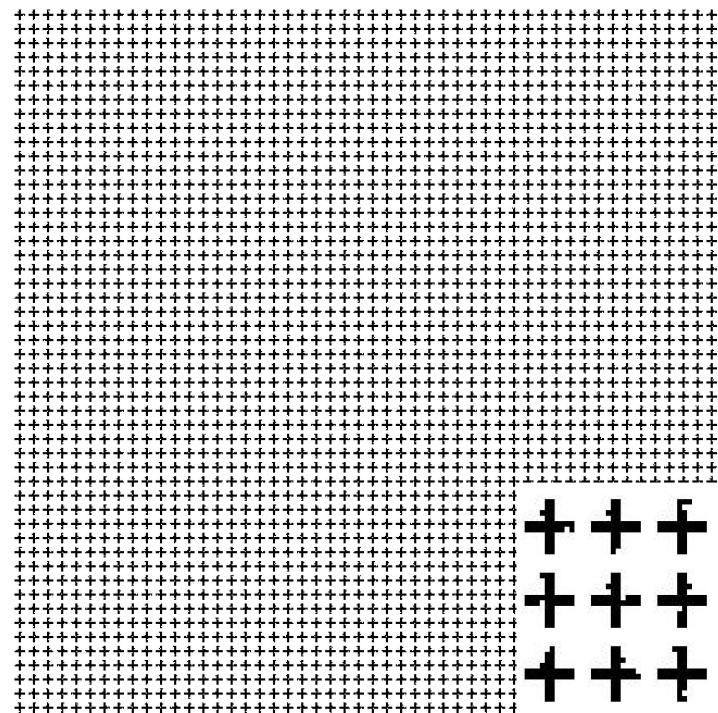

Figure 3.32. Simulated systematic error. $2048 \times 2048$ pixel array of 50 rows and columns of crosses, each one distorted by the removal of a random square which is relocated onto the top arm, introducing a bias. The inset shows a closeup of the crosses and is not part of the CIP input.
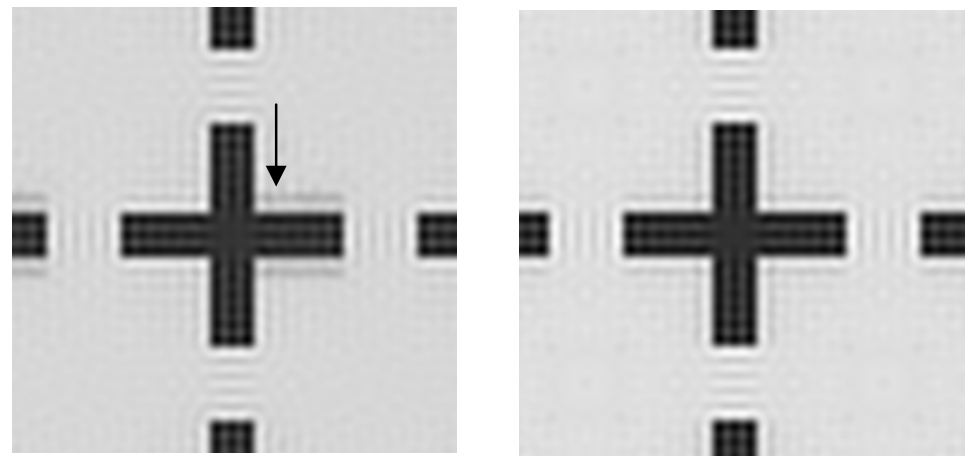

Figure 3.33. (left) Periodic motifs of biased cross image after $p l$ enforcement; note banding around right arm of cross; (right) same image after $\mathrm{p} 4 \mathrm{~mm}$ enforcement, all arms of the cross are symmetrical. 


\subsection{CIP restoration of an image with systematic error}

Figures 3.34 and 3.35 were taken by a PSIA (Park Systems) XE-120 AFM in the laboratory of Prof. Andres La Rosa at Portland State University. The images are of a Nanosurf BT00250 calibration grid intended for AFM use. The "islands" on the grid form a square lattice with a periodicity of $10 \mu \mathrm{m}$ and have a measured height of 119 nanometers (nominal $100 \mathrm{~nm}$ ). The images were taken at approximately the same time, at room temperature and with the sample exposed to the atmosphere.

The instrument has a proprietary hardware closed-loop feedback system for the piezoelectric scanners in the $\mathrm{x}$ and $\mathrm{y}$ directions (it also has one for the $\mathrm{z}$-direction piezo). "Closed-loop" is a generic term from control theory that refers to a system that uses feedback from the output to adjust input states, usually to maintain or stabilize a value. Piezoelectric crystals have natural hysteresis; thus an AFM raster scan that is intended to be rectangular can be an irregular quadrilateral absent correction. The feedback system is designed to correct this behavior.

Figure 3.34 is an image of the calibration grid made with the $x-y$ feedback system on. Figure 3.35 was made with the feedback system deliberately turned off so as to create an image influenced by hysteresis.

No special efforts were made to level or otherwise prepare the sample; the intent was to create "quick and dirty" images so as to assess the value of CIP. The color difference from top to bottom across both images, which shows the $\mathrm{z}$ coordinate is changing from top to bottom, is evidence of sample tilt. 


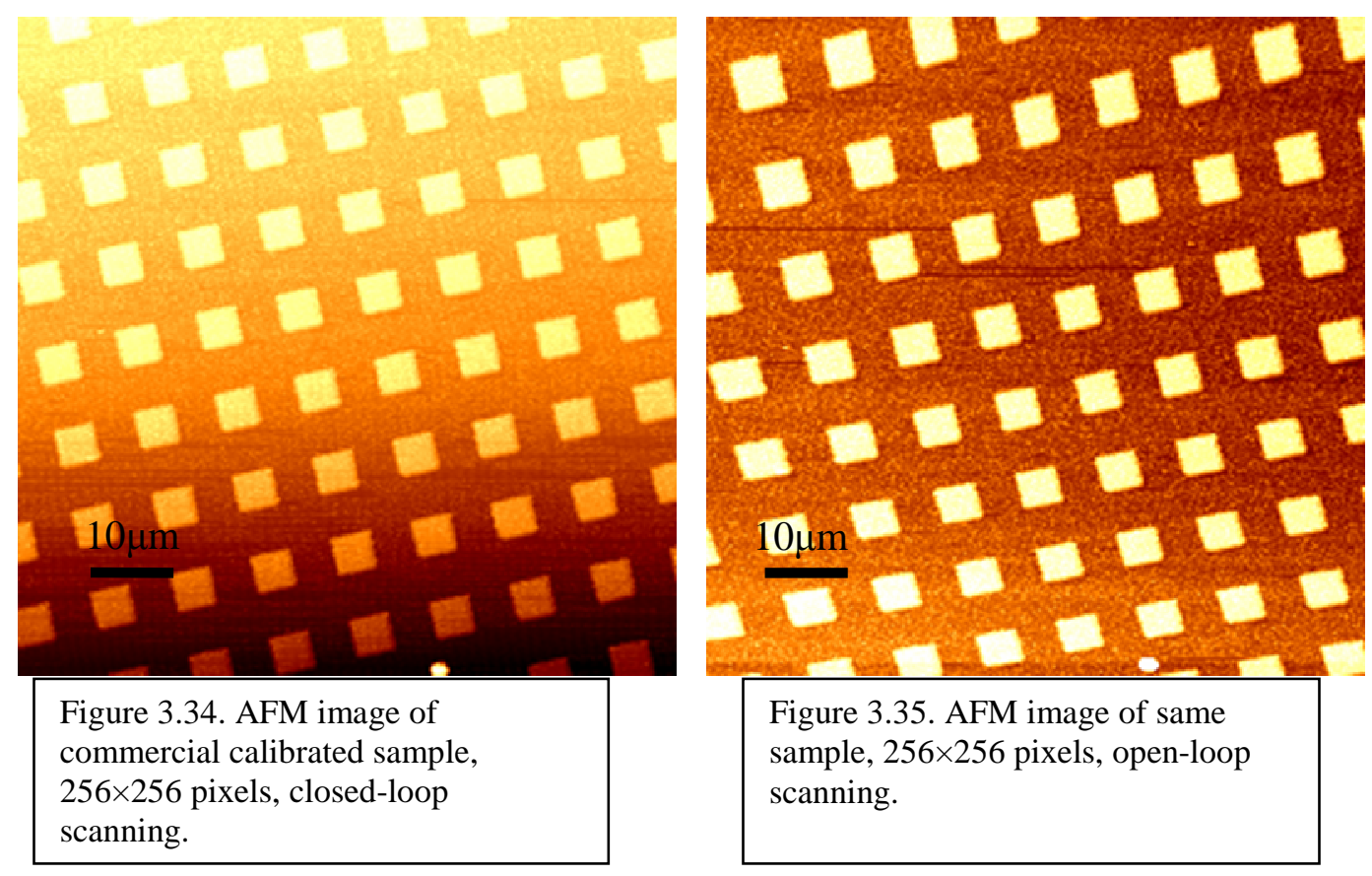

Both images were processed with CIP. The closed-loop image in Figure 3.34,

which is visibly more symmetric to the human eye, was enforced with $p 4 m m$ symmetry.

The residuals were low and the angle between the two reciprocal axes was 90.1 degrees.

Figure 3.36 shows the Fourier transform and figure 3.37 shows the residuals for the various plane groups and a $14 m m$ enforced image.

The closed-loop feedback of this instrument does in fact appear effective in correcting for hysteresis in that there is no visual nonlinearity in the $\mathrm{x}-\mathrm{y}$ direction before or after CIP, but note that the $p 4 m m$ enforced image also eliminates the sample tilt. 

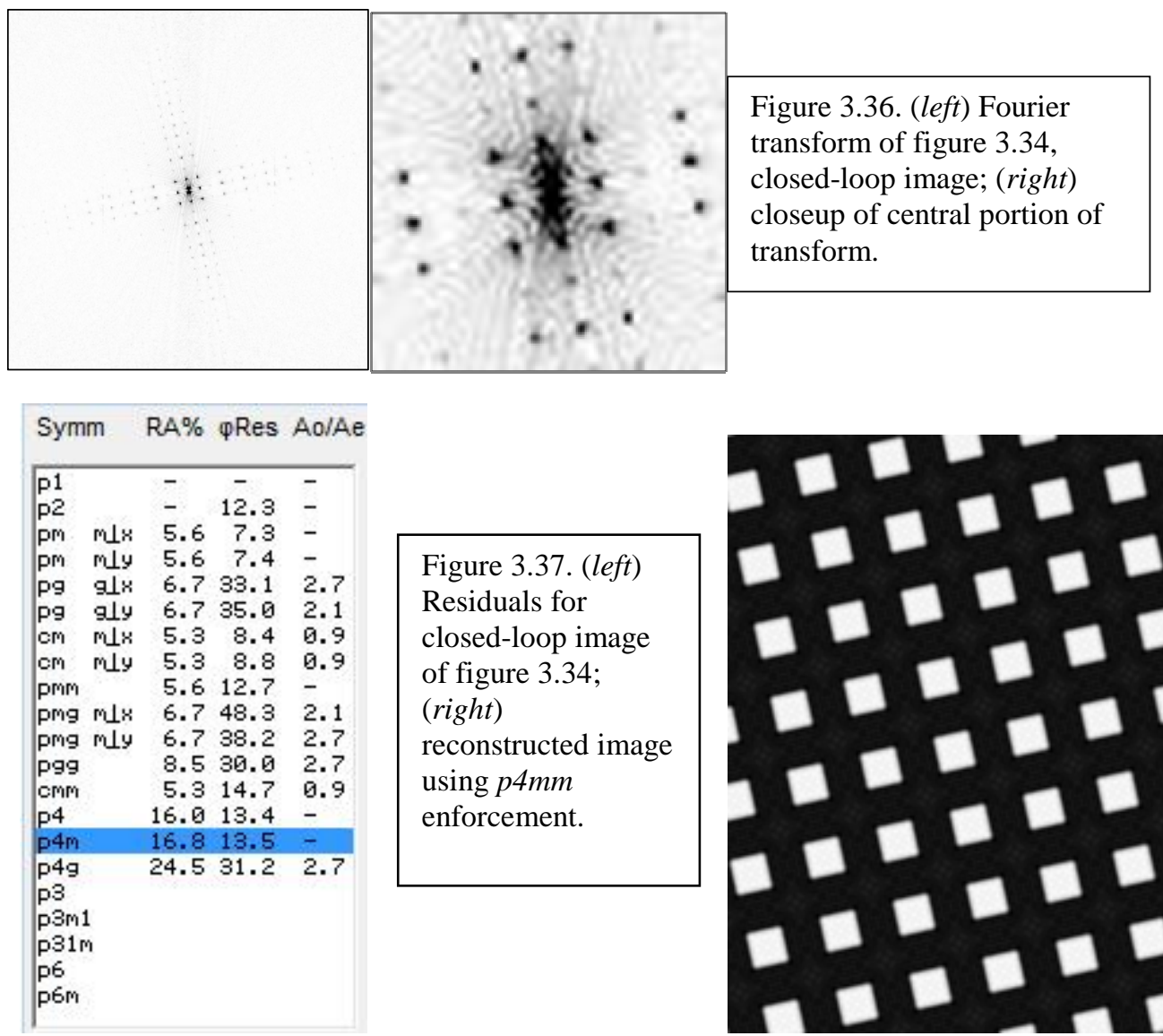

Figure 3.37. (left)

Residuals for

closed-loop image

of figure 3.34;

(right)

reconstructed image

using $\mathrm{p} 4 \mathrm{~mm}$

enforcement.

Processing the image with induced systematic error, Figure 3.35, required manual indexing of the Fourier transform due to blurring of the spots caused by the asymmetries of the image. That transform and a closeup of the central portion are shown in Figure 3.38 showing the blurring of the spots. 
The final result of CIP applied to Figure 3.35 is shown in Figure 3.39, which shows the residuals and a $p 4 m m$ enforced reconstruction of the image. The residuals for p4mm are higher than for the closed-loop image, as to be expected: RA\% of 20.6 vs. 16.8, and $\varphi$ Res of 20.7 vs. 13.5, a consequence of the image asymmetry. The enforcement of $\mathrm{p} 4 \mathrm{~mm}$ symmetry is seen to successfully remove the artificially induced systematic error.

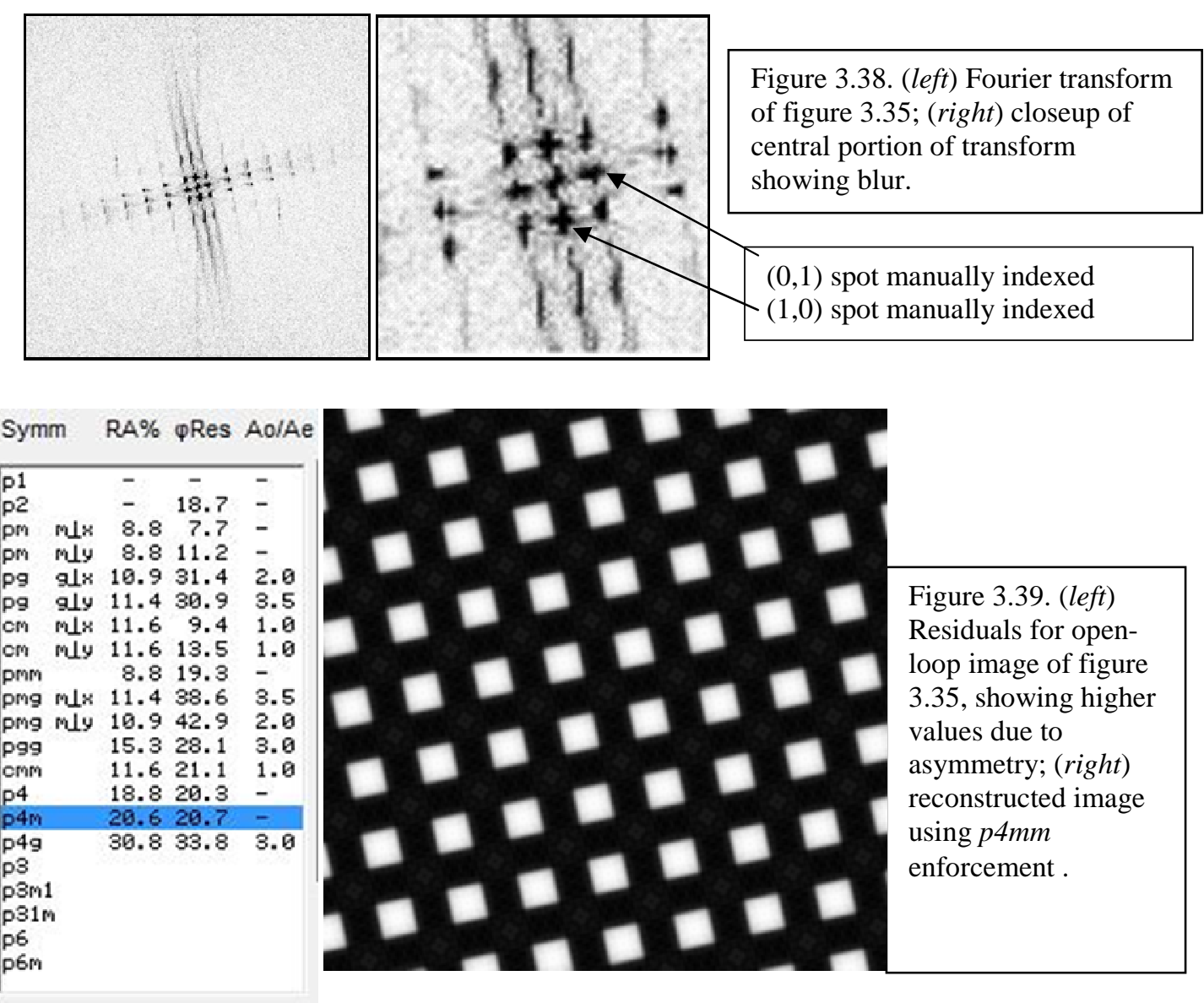




\section{CIP as a technique for ascertaining the SPM tip}

Given a sample of known 2D periodicity, comparing the Fourier coefficients of an image of this sample before and after correction by symmetry enforcement offers a unique opportunity to calculate the distortions induced by the instrument.

The image that is obtained by any real instrument is not perfect. There is noise as well as systematic distortions such as piezo hysteresis, bow, and drift. These problems are well known in SPM and there are existing techniques for correcting them. CIP applied to a known periodic sample is a unique and novel way to ascertain and correct for the distortions in a given experimental configuration.

The effect of the instrument on a sample can be summarized in the point spread function (PSF), a term from optics that is applicable to any imaging system. If we define images and objects as $2 \mathrm{D}$ arrays of intensity, the image of an object is the convolution of that object with the PSF of the imaging instrument. The convolution theorem says therefore that Fourier transforms of the object and PSF can be multiplied to give the Fourier transform of the image.

The idea here (shown mathematically in equations 4.1 through 4.6) is that the PSF can be obtained by dividing the Fourier transform of the image by that of the object and applying an inverse Fourier transform to the result.

The idea of deconvoluting an SPM image to obtain tip information is not new. In 1990 Snyder et al [19] investigated the causes of broadening and skewing of STM images caused by tip size and asymmetry. In particular they simulated images of 
graphite that modeled elliptical tips with the major axis of the ellipse inclined at various angles to the scanning direction.

They noticed that their simulations strongly resembled certain anomalous real images of graphite. They chose an actual image with skew and found that if they deconvoluted it with a particular elliptical tip function they produced a new image that looked like graphite. They then inferred that the actual tip must have had this elliptical geometry.

We propose a systematic approach for tip inference involving CIP of images of a known calibration standard followed by PSF calculation. To the best of our knowledge this has never been done before.

An ideal STM tip is a mathematical point; a slender cone that is axially symmetric and narrows to a single orbital at the tip, with all tunneling occurring through this one atom in a radially symmetric fashion (possibly an s-orbital, more commonly a $d$ orbital.) An ideal AFM tip would be similarly shaped (although no tunneling takes place through the apex atom). Any real tip departs from this perfectly symmetric ideal. Tips can be double, multiple, or "blunt" (irregularly shaped) on a scale ranging from subatomic to macroscopic. A clear double-tip image is shown in Figure 4.1.

Note that the entire SPM instrument introduces distortion - nonlinear piezos, nonperfect amplifiers in the electronics, etc. This thesis refers to the entire distortion as the "effective tip" and will attempt to mathematically summarize the distortions as the point spread function (PSF) of the tip. 


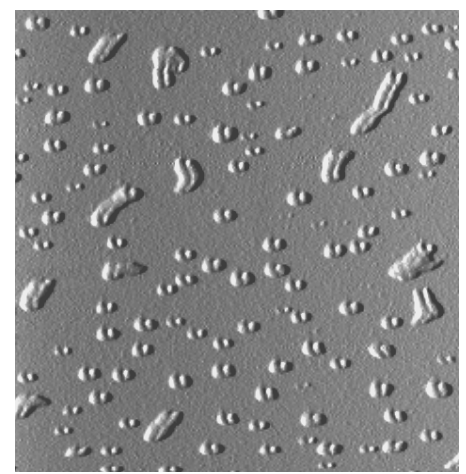

Figure 4.1 Classic image of DNA and debris showing a double tip artifact;

Weizmann Institute of

Science,

www.weizmann.ac.il
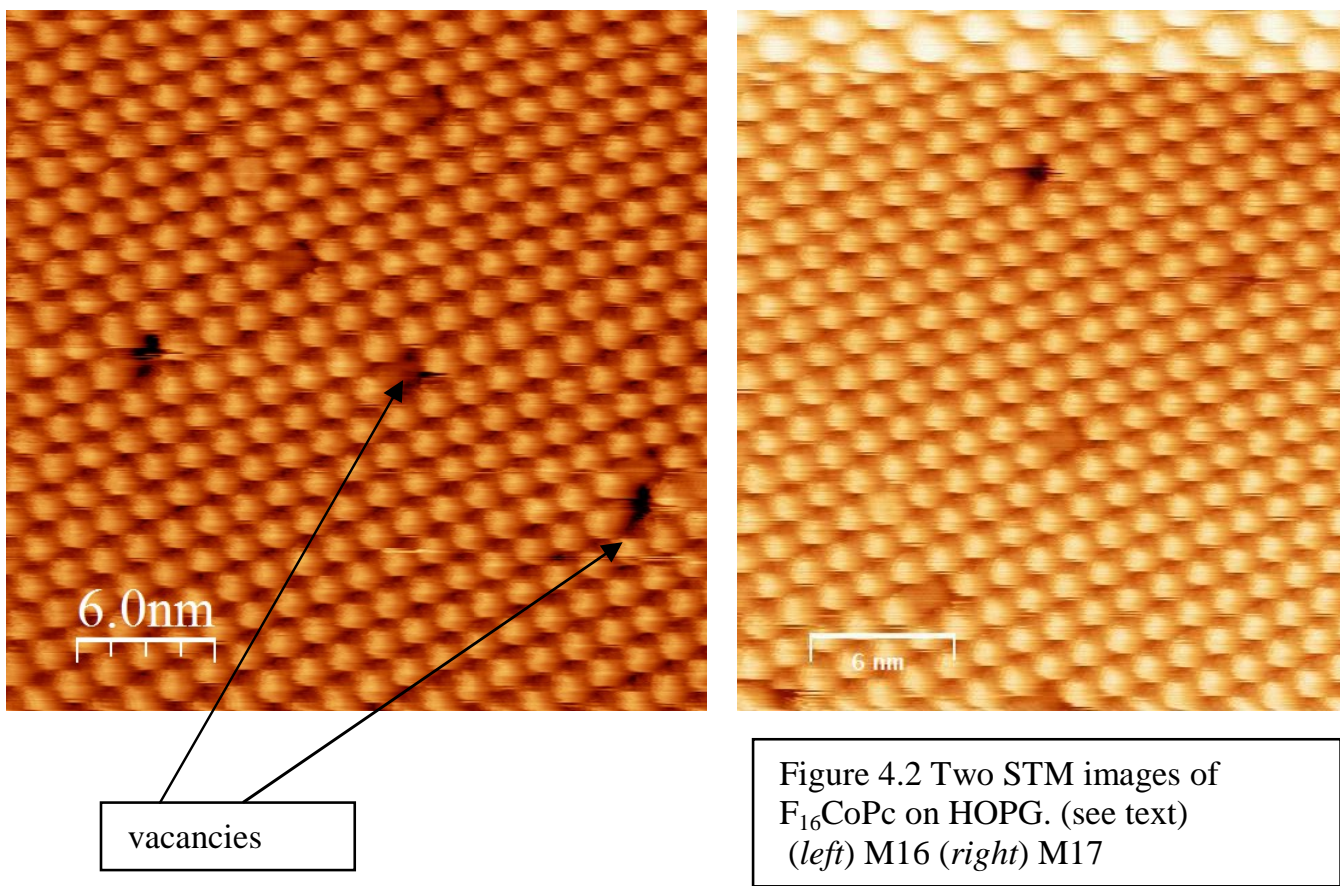

The images to be studied are called "M16" and "M17" according to the nomenclature used in the laboratory. They are raw 512 by 512 pixel STM images of a layer of fluorinated cobalt phthalocyanine on highly oriented pyrolytic graphite (HOPG). They were taken at the Technical Institute of Chemnitz with an Omicron STM in constant current mode with a +1.0 volt bias on the tungsten tip, tunneling current of $0.15 \mathrm{nA}$, under ultrahigh vacuum at $30 \mathrm{~K}$. Under certain bias conditions (per conversation from K.W. Hipps, Washington State University to P. Moeck) organic 
molecules on HOPG are nearly invisible to STM. Reference [28], coauthored by Hipps, describes a similar phenomenon involving a nickel porphyrin on HOPG. When M16 and M17 are analyzed, their plane symmetry very much resembles that of HOPG, although the Fourier transforms of the images do in fact have higher-order coefficients, which is not the case for STM images of pure graphite (see Fig. 3.4) - thus some information about the organic molecule is present. They were taken during one experimental session and are, to the best of our knowledge, taken under very similar experimental conditions from different areas of the same sample.

For the purpose of ascertaining the point spread function (which essentially comprises the effective tip) one needs a calibrated standard, ideally with high plane symmetry and able to encompass a large number of unit cells in an image.

HOPG with a surface layer that is a regular array of highly symmetric molecules may meet these requirements.

Visual inspection of the above images shows vacancies, noise, some bowing, and in the case of M17 a scanning discontinuity a few lines from the top. The vacancies provide evidence of true molecular resolution (and are also evidence that the image is not pure HOPG, which seldom shows vacancies). If one assumes that the underlying sample of the above two images has p3ml symmetry, with the exception of the vacancies any departure from that symmetry must be a result of the combined imperfections of the instrument. The PSF is a mathematical expression of this symmetry departure. 
As seen in equations 2.11 and 2.12, an image is the convolution of the sample surface feature function with the effective tip function.

$$
i(\boldsymbol{r})=o(\boldsymbol{r}) \otimes p(\boldsymbol{r})
$$

where $r$ is a 2D vector in real space, $i$ is the image, $o$ is the object (sample), and $p$ is the effective tip function. The $r$ vector is theoretically continuous but given a pixellated image will be part of a discrete set of $2 \mathrm{D}$ vectors. By the convolution theorem, the Fourier transformation of the above functions changes the convolution operator to a simple multiplication in Fourier space. This allows algebraic manipulation to recover one of the right-side functions if the other (and the left side) is known.

$$
I\left(g^{*}\right)=O\left(g^{*}\right) P\left(g^{*}\right)
$$

where $g^{*}$ is a vector in reciprocal space whose components are multiples of the reciprocal unit vectors $\boldsymbol{a}^{*}$ and $\boldsymbol{b}^{*}$. Similarly to the $r$ vector in real space, the $g^{*}$ vector is theoretically continuous but is actually exists only at discrete intervals, both because it is the transformation of a finite $2 \mathrm{D}$ array and more importantly because to the extent that $o(r)$ is $2 \mathrm{D}$ periodic the amplitude component of its Fourier transform is a set of spots.

It is customary to refer to the coordinates in $2 \mathrm{D}$ reciprocal space with the variables $H$ and $K$. Rewriting (4.2) above for clarity, we obtain:

$$
I_{\text {raw }}(H, K)=O_{\text {sample }}(H, K) \bullet P_{\text {tip }}(H, K)
$$

Given $I_{\text {raw }}(H, K)$, knowledge of either $O_{\text {sample }}(H, K)$ or $P_{\text {tip }}(H, K)$ allows solution of the other. In particular, 


$$
P_{\text {tip }}(H, K)=I_{\text {raw }}(H, K) / O_{\text {sample }}(H, K)
$$

If the sample is a known calibration standard, preferably highly symmetrical, one can use this prior knowledge upon any SPM observation of this standard that appears reasonably close to reality. The already-known symmetry can be enforced, and these enforced Fourier coefficients can be taken as $O_{\text {sample }}(H, K)$.

The comparison of observed Fourier coefficients $I$ with theoretical Fourier coefficients $O$ is an opportunity to calculate $P_{t i p}(H, K)$. The inverse Fourier transformation of $P$ will result in the point spread function $p_{t i p}(x, y)$.

The functions $I$ and $O$ are complex-valued functions of a discrete 2D array of integers. We seek $p_{t i p}(x, y)$ ideally as a two-dimensional continuous function but our intermediate result $P_{t i p}(H, K)$ only has as many points in its domain as there are non-zero Fourier coefficients $I_{\text {raw }}(H, K)$ and $O_{\text {sample }}(H, K)$. The result of the inverse Fourier transform that takes $P$ to $p$ should accurately describe the tip function.

We treat M16 as such a calibration standard, of known symmetry and periodic on a molecular scale, because it is seen that the symmetry of the graphite is largely shown in the Fourier coefficients. The effect of molecular vacancies will be seen to average out. Given the prior knowledge that the sample is graphite and its plane group is $p 3 m 1$ one can enforce that symmetry and equate:

$$
O_{\text {sample }}(H, K)=I_{\text {symmetry_enforced }}(H, K)
$$

Thus after the enforcing the plane symmetry of the known calibration sample, one can rewrite relation (4.4) as 


$$
P_{\text {tip }}(H, K)=I_{\text {raw }}(H, K) / I_{\text {symmetry_enforced }}(H, K)
$$

and thus obtain the Fourier transform of the point spread function for a particular scanning probe tip (actually the tip+instrument unit) and a particular set of experimental conditions of the SPM, provided $I$ is of a known calibration standard.

This estimate for $P_{t i p}(H, K)$ can now be used directly for correcting images of unknown samples that are recorded under the same conditions, including the same tip, as the image taken of the calibration sample. The respective relation is

$$
I_{\text {corrected_unknown }}(H, K) \approx I_{\text {raw_unknown }}(H, K) / P_{\text {tip }}(H, K)
$$

\subsection{Calculations on image M16}
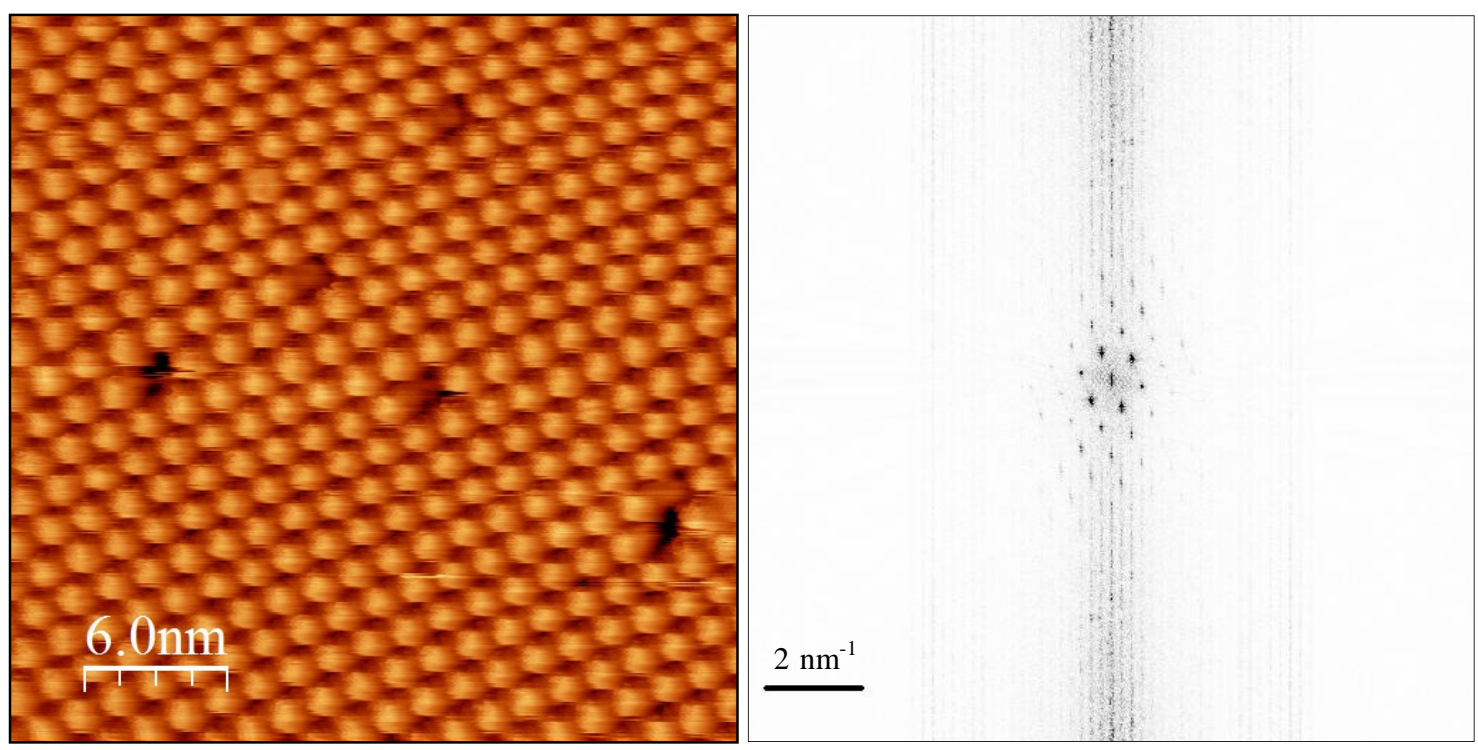

Figure 4.3. M16 on the left, $512 \times 512$ pixels, 18 (vertical) x 20 (horizontal) unit cells. Its twodimensional Fourier transformation (black/white reversed for visibility) on the right, taken from a 512 pixel diameter circle centered on the image. 
The image in the right half of figure 4.3 is the Fourier transformation of M16. The vertical banding is a consequence of the visible horizontal raster scan lines in the raw image. The spots seen at the center derive from the periodic elements in the sample. They are clearly in an approximate hexagonal arrangement; i.e. the angle between lines joining them is approximately 60 degrees. This is evidence that the proper plane group for this sample is one of the hexagonal groups: $p 3, p 3 m 1, p 31 m, p 6$, or $p 6 m m$.

Figure 4.4 shows the next step in CIP as implemented in CRISP: selection of the reciprocal lattice. This is the same Fourier transform as figure 4.3 but with arrows overlaid showing the direction and unit size of the $H$ and $K$ axes. This lattice selection was made automatically by CRISP and was not overridden as it clearly matches the
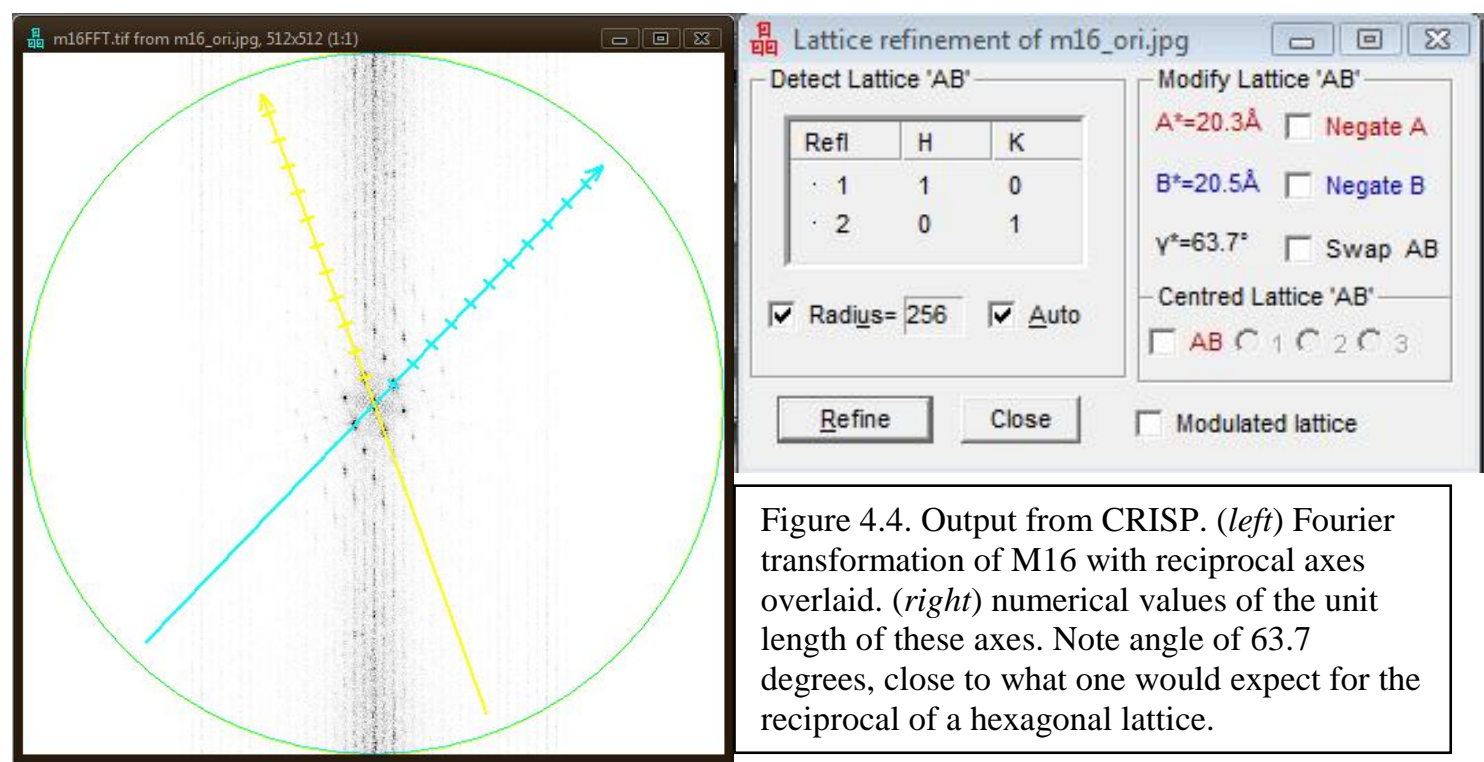

Figure 4.4. Output from CRISP. (left) Fourier transformation of M16 with reciprocal axes overlaid. (right) numerical values of the unit length of these axes. Note angle of 63.7 degrees, close to what one would expect for the reciprocal of a hexagonal lattice.

actual spacing of the spots. The numerical values shown for the reciprocal length of the axes is calibration dependent; the default settings for CRISP are what are used since the 
ratio of the reciprocal lengths is what is significant for determining the plane group rather than absolute values.

This confirms the visual observation that the axes are approximately 60 degrees apart and as seen in figure 4.5 CRISP tests just the hexagonal plane groups (as well as p2 which is always calculated, and $p 1$ which is translational averaging.)

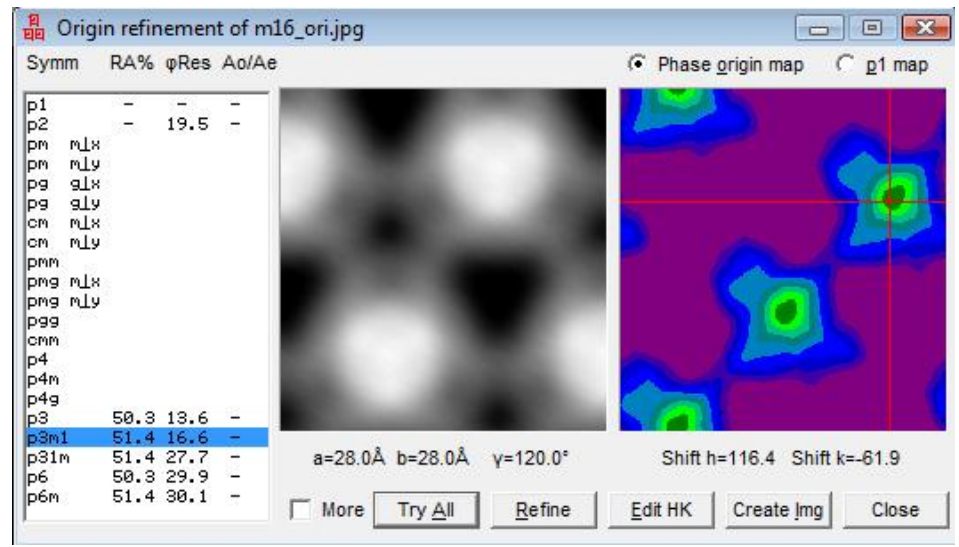

Figure 4.5. Left panel contains the list of plane groups with their residuals. Note that only the five hexagonal groups are tested.

The software has selected $p 3 m 1$ as the most likely plane group. The residuals for this group are slightly higher than those for $p 3$, but because the multiplicity of $p 3 m 1$ is 6 while that of $p 3$ is only 3 , there is a greater "quantity of symmetry" in $p 3 m 1$ and it is the better choice.

Note that CRISP has selected " $\mathrm{a}=28.0 \AA, \mathrm{b}=28.0 \AA$ ” as the dimensions of the reciprocal lattice unit vectors. The " $\AA$ " shown as the dimension of the output is should be interpreted as inverse pixels. The size is consistent with a 512 pixel lattice with 18 periods $(28 \approx 512 / 18)$.

We now enforce that symmetry and look at the Fourier coefficients before and after enforcement in table 4.1. Amplitudes are denoted in scalar units, with a dynamic 
range of up to 10000; this table is limited to coefficients with enforced amplitudes greater than 200 and contains 24 entries. The ratio of the amplitudes is a meaningful quantity when calculating the inverse Fourier transform; the actual value is irrelevant.

\begin{tabular}{|c|c|c|c|c|c|}
\hline \multicolumn{2}{|c|}{$\begin{array}{r}\text { Coordinates (reciprocal } \\
\text { space) }\end{array}$} & \multicolumn{2}{|c|}{$\begin{array}{c}\text { Fourier amplitudes (arbitrary } \\
\text { units) }\end{array}$} & \multicolumn{2}{|c|}{ Fourier phases (degrees) } \\
\hline$H$ & $K$ & observed & $p 3 m 1$ enforced & observed & $p 3 m 1$ enforced \\
\hline 0 & 1 & 10000 & 7565 & -10 & -10 \\
\hline 1 & 0 & 9198 & 7565 & 10 & 10 \\
\hline 1 & -1 & 3497 & 7565 & -9 & -10 \\
\hline 0 & 2 & 1550 & 850 & 38 & 57 \\
\hline 2 & 0 & 690 & 850 & -148 & -57 \\
\hline 2 & -2 & 309 & 850 & -83 & 57 \\
\hline 1 & 1 & 2072 & 1132 & -167 & 180 \\
\hline 1 & -2 & 788 & 1132 & -14 & 180 \\
\hline 2 & -1 & 537 & 1132 & -71 & 180 \\
\hline 1 & 2 & 1374 & 561 & 87 & 106 \\
\hline 1 & -3 & 138 & 561 & 118 & -106 \\
\hline 2 & 1 & 1147 & 561 & -116 & -106 \\
\hline 2 & -3 & 92 & 561 & 47 & 106 \\
\hline 3 & -1 & 409 & 561 & 173 & -106 \\
\hline 3 & -2 & 207 & 561 & 46 & 106 \\
\hline 1 & 3 & 906 & 237 & -175 & -155 \\
\hline 1 & -4 & 137 & 237 & 27 & 155 \\
\hline 3 & 1 & 258 & 237 & 122 & 155 \\
\hline 3 & -4 & 43 & 237 & -84 & -155 \\
\hline 4 & -1 & 61 & 237 & 130 & 155 \\
\hline 4 & -3 & 15 & 237 & 156 & -155 \\
\hline 2 & 2 & 592 & 238 & 176 & 180 \\
\hline 2 & -4 & 86 & 238 & 23 & 180 \\
\hline 4 & -2 & 36 & 238 & -56 & 180 \\
\hline
\end{tabular}

The table columns marked "observed" are the Fourier transform of data gathered directly from the image. As discussed earlier, the transform results in a two-dimensional matrix of complex numbers. The amplitudes in the table are the magnitudes of those 
complex numbers, where the magnitude of a complex number $x+i y$ is $\sqrt{x^{2}+y^{2}}$. If one expresses the complex number $x+i y$ in the form $r e^{i \theta}$, then the magnitude is $r$.

The phases are the argument of the complex number, defined as the branch (180,180] expressed in degrees of $\operatorname{arctangent}(y / x)$ or alternatively as $\theta$ if the number is expressed in the form $r e^{i \theta}$. The observed phase depends upon the origin selection, which is initially set to be the center of the real-space area selected for analysis.

The Fourier amplitudes are absolute (although their unit is arbitrary) but the Fourier phases are relative to the selected origin. An origin shift shifts all the phases according to the formula $\varphi_{\text {new }}=\varphi_{\text {old }}+360^{\circ}(h x+k y)$ where $h, k$ are the indexes of the coefficient and $x, y$ is the shift in fractional coordinates [26].

With the exception of $p 1$, each of the 17 plane groups has either a specific location or an axis (glide or mirror) along which the origin must be defined. The initial origin at the center of real space is unlikely to be at this location. Therefore for each plane group a CIP program tests each point in the unit cell as a potential origin. The Fourier coefficients are calculated at each tentative shifted position, and the position (or arbitrary location along a mirror/glide line) that has the lowest residuals (see equations 2.7 and 2.8) is chosen as the origin.

The columns marked "enforced" are the end result of crystallographic image processing. Various Fourier coefficients are grouped together with amplitudes and phases averaged. The groupings depend on the plane group selected. In the case of p3ml, these coefficients are related: $(h k)(k-h-k)(-h-k h)(k h)$. Additional relationships 
exist because the relation between $(h k)$ and $(k h)$ in particular means that the values for $k$ and $h$ can be permuted. For example, let $h=1$ and $k=3$ and note in rows 16 though 21 of the table that these coefficients: $\left(\begin{array}{ll}1 & 3\end{array}\right)\left(\begin{array}{ll}1 & -4\end{array}\right)\left(\begin{array}{ll}3 & 1\end{array}\right)\left(\begin{array}{ll}3 & -4\end{array}\right)\left(\begin{array}{l}4 \\ -1\end{array}\right)(4-3)$ are all grouped together.

These grouped coefficients have the same enforced amplitude and enforced phases that are either the same or differ by 180 degrees. Certain groups of coefficients, those that contain values of $h$ and $k$ in which $h=k$ restrict the phase to 0 or 180 degrees, again a requirement of $p 3 m 1$.

The two columns labeled "enforced" constitute a new set of Fourier coefficients that, when inverse Fourier transformed, will display a "corrected" image of the original sample. See figures 4.6 and 4.7 which show the corrected image, first with the 24 strongest coefficients shown in the table, then with the 53 coefficients with amplitudes greater than or equal to 50 (in arbitrary units)

Given these coefficients we can now compute the point spread function of the effective microscope tip by comparing the corrected coefficients to the raw coefficients. This is a simple division of complex numbers that takes two complex functions of the two dimensional integer array $(H K)$ and yields a third complex function of $(H K)$. Using equation 4.6,

$$
P_{\text {tip }}(H, K)=I_{\text {raw }}(H, K) / I_{\text {symmetry_enforced }}(H, K)
$$

we get table 4.2 (in which the result is expressed as the reciprocal of $P_{\text {tip }}$ for ease of later calculation): 


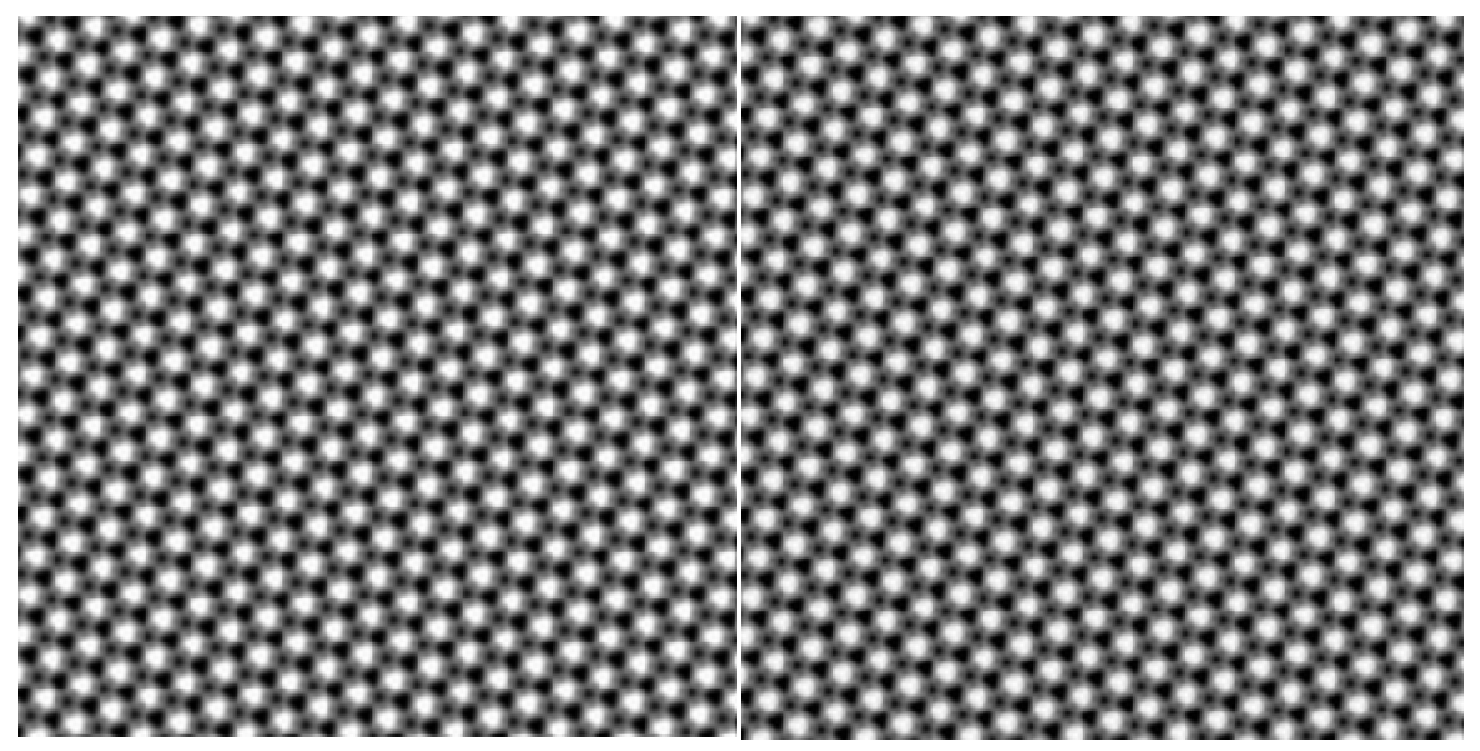

Figure 4.6. Corrected image of M16 enforcing p3ml symmetry using 24 coefficients with highest amplitude.

Figure 4.7. Corrected image of M16 enforcing p3ml symmetry using 53 coefficients.

\begin{tabular}{|c|c|c|c|c|c|c|c|}
\hline \multicolumn{2}{|c|}{$\begin{array}{r}\text { Coordinate (Fourier } \\
\text { space) }\end{array}$} & \multicolumn{2}{|c|}{$\mathrm{I}_{\text {raw }}(H, K)$} & \multicolumn{2}{|c|}{$I_{\text {symmetry_enforced }}(H, K)$} & \multirow{2}{*}{\multicolumn{2}{|c|}{$\begin{array}{c}1 / P_{\text {tip }}(H, K) \\
\text { (in } r e^{i \vartheta} \text { format) }\end{array}$}} \\
\hline$H$ & $K$ & \multicolumn{4}{|c|}{ (expressed in $x+i y$ format) } & & \\
\hline 0 & 1 & 9848.1 & -1736.5 & 7450.1 & -1313.6 & 0.757 & 0 \\
\hline 1 & 0 & 9058.3 & 1597.2 & 7450.1 & 1313.6 & 0.822 & 0 \\
\hline 1 & -1 & 3453.9 & -547.1 & 7450.1 & -1313.6 & 2.163 & -1 \\
\hline 0 & 2 & 1221.4 & 954.3 & 462.9 & 712.9 & 0.548 & 19 \\
\hline 2 & 0 & -585.2 & -365.6 & 462.9 & -712.9 & 1.232 & 91 \\
\hline 2 & -2 & 37.7 & -306.7 & 462.9 & 712.9 & 2.751 & 140 \\
\hline 1 & 1 & -2018.9 & -466.1 & -1132.0 & 0.0 & 0.546 & -13 \\
\hline 1 & -2 & 764.6 & -190.6 & -1132.0 & 0.0 & 1.437 & -166 \\
\hline 2 & -1 & 174.8 & -507.7 & -1132.0 & 0.0 & 2.108 & -109 \\
\hline 1 & 2 & 71.9 & 1372.1 & -154.6 & 539.3 & 0.408 & 19 \\
\hline 1 & -3 & -64.8 & 121.8 & -154.6 & -539.3 & 4.065 & 136 \\
\hline 2 & 1 & -502.8 & -1030.9 & -154.6 & -539.3 & 0.489 & 10 \\
\hline 2 & -3 & 62.7 & 67.3 & -154.6 & 539.3 & 6.098 & 59 \\
\hline 3 & -1 & -406.0 & 49.8 & -154.6 & -539.3 & 1.372 & 81 \\
\hline 3 & -2 & 143.8 & 148.9 & -154.6 & 539.3 & 2.710 & 60 \\
\hline 1 & 3 & -902.6 & -79.0 & -214.8 & -100.2 & 0.262 & 20 \\
\hline
\end{tabular}




\begin{tabular}{|r|r|r|r|r|r|r|r|}
\hline 1 & -4 & 122.1 & 62.2 & -214.8 & 100.2 & 1.730 & 128 \\
\hline 3 & 1 & -136.7 & 218.8 & -214.8 & 100.2 & 0.919 & 33 \\
\hline 3 & -4 & 4.5 & -42.8 & -214.8 & -100.2 & 5.512 & -71 \\
\hline 4 & -1 & -39.2 & 46.7 & -214.8 & 100.2 & 3.885 & 25 \\
\hline 4 & -3 & -13.7 & 6.1 & -214.8 & -100.2 & 15.800 & 49 \\
\hline 2 & 2 & -590.6 & 41.3 & -238.0 & 0.0 & 0.402 & 4 \\
\hline 2 & -4 & 79.2 & 33.6 & -238.0 & 0.0 & 2.767 & 157 \\
\hline 4 & -2 & 20.1 & -29.8 & -238.0 & 0.0 & 6.611 & -124 \\
\hline
\end{tabular}

\subsection{Calculations on image M17 using the PSF from M16}

Now that we have calculated the presumed PSF of the tip by enforcing $p 3 m 1$ symmetry upon image M16 and comparing those coefficients with the raw image coefficients, one can examine image M17 (figure 4.8), taken at nearly the same time and under the same experimental conditions, and correct for the distortions of M17 introduced by the tip + instrument combination by dividing those coefficients by the Fourier transform of the M16 PSF using formula 4.7

$$
I_{\text {corrected_unknown }}(H, K) \approx I_{\text {raw_unknown }}(H, K) / P_{\text {tip }}(H, K)
$$

\subsubsection{Zero values in the PSF}

We now must deal with the fact that since $P_{t i p}(H, K)$ appears in the denominator of this expression, if $P_{t i p}=0$ for any particular $H, K$ pair the expression will not be defined. This will occur for any $H, K$ for which the Fourier amplitude of the observation is zero (since $\left.P_{\text {tip }}(H, K)=I_{\text {raw }}(H, K) / I_{\text {symmetry_enforced }}(H, K)\right)$. One possible solution is to add a Wiener-filter type step to the process, by adding a small constant to each observation amplitude, simulating a bias, thus guaranteeing a finite solution [29]. 
There are two logical places in the process where this can be done: either in the calculation of the PSF itself:

in which case

$P_{\text {tip }}(H, K)=I_{\text {raw }}(H, K) / I_{\text {symmetry_enforced }}(H, K)$

becomes

$P_{\text {tip }}(H, K)=\left(I_{\text {raw }}(H, K)+k_{\text {bias }}\right) / I_{\text {symmetry_enforced }}(H, K)$

that guarantees that $P_{t i p}(H, K)$ will be nonzero since $I_{\text {raw }}(H, K)$ is nonnegative, or later, in the calculation of $I_{\text {corrected_unknown }}(H, K)$

in which case

$I_{\text {corrected_unknown }}(H, K) \approx I_{\text {raw_unknown }}(H, K) / P_{\text {tip }}(H, K)$

becomes

$I_{\text {corrected_unknown }}(H, K) \approx I_{\text {raw_unknown }}(H, K) /\left(P_{\text {tip }}(H, K)+k_{\text {bias }}\right)$

that guarantees that $I_{\text {corrected_unknown }}(H, K)$ will remain finite.

The second method involving only the calculation of $I_{\text {corrected_unknown }}(H, K)$ will be used here since it leaves the PSF unchanged for other purposes (such as real-space visualization of the tip) and since it is expected that the calculation of $I_{\text {corrected }}$ $\operatorname{unknown}(H, K)$ is an approximation anyway.

An alternative approach that will not be further pursued here would be to introduce a nonzero random noise $k_{\text {noise }}$ instead of $k_{\text {bias }}$, which would eliminate the problem of biasing all coefficients in the same direction. 


\subsubsection{Production of corrected M17 coefficients}

The Fourier transform of M17 will of course consist of a two dimensional array of complex numbers. Although all coefficients in the array contribute to the final image that is produced after an inverse Fourier transform, the higher the amplitude of the coefficient, the greater the visual intensity of that particular contribution in real space. In practice, our experience has been that the 20 to 25 highest amplitude coefficients can
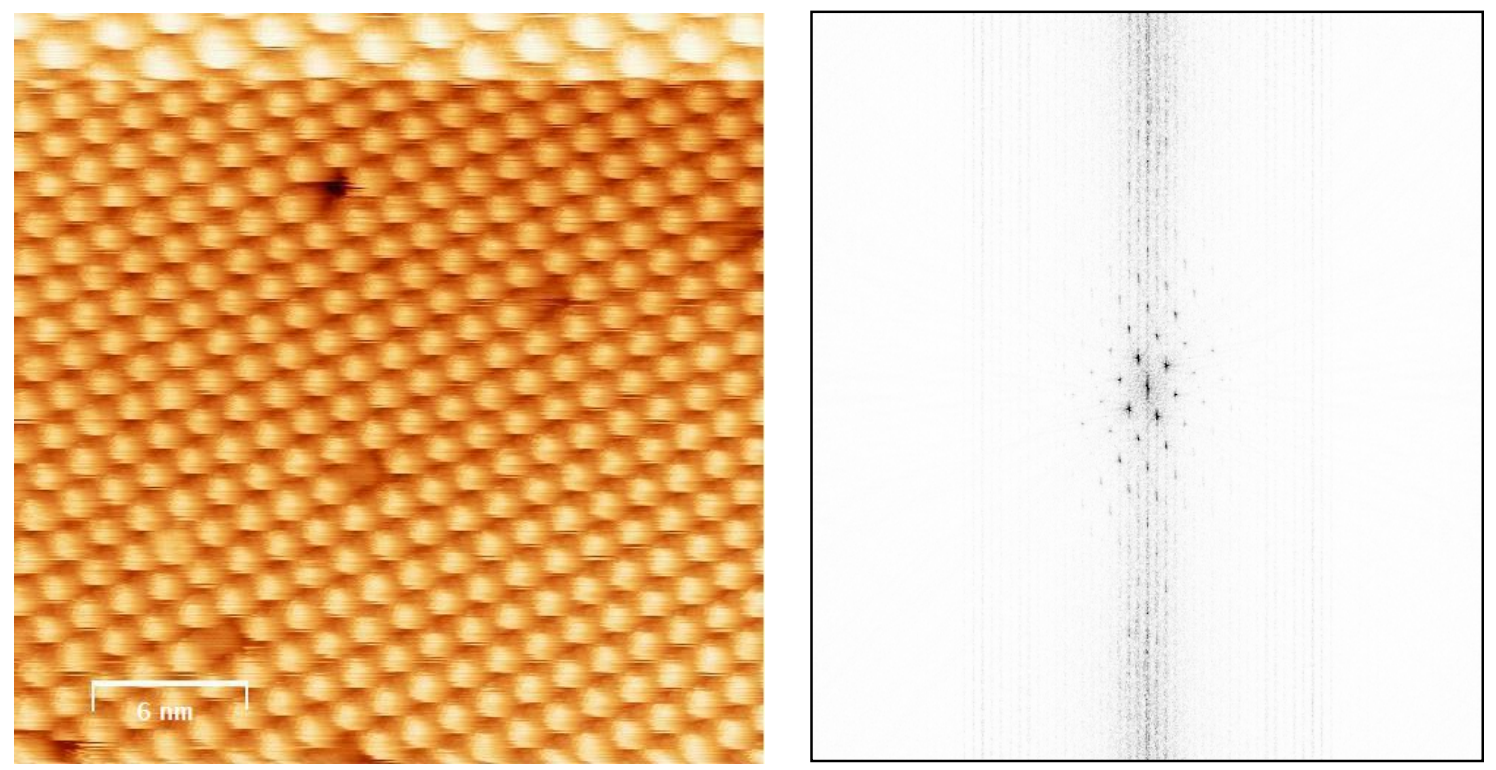

Figure 4.8. M17 on the left, its Fourier transform on the right based upon a 512-pixel diameter circle centered on the image.

produce an image indistinguishable to the eye from an image with more coefficients.

It seems logical then to concentrate on the strongest coefficients of the image being worked on; we are using the 24 strongest for ease of calculation. This particular 
set of $24(H, K)$ indices will not be the same for every image, and the PSF calculated from the image of the calibrated sample should contain a large enough number of coefficients to insure there will be a $P_{\text {tip }}(H, K)$ value for each $I_{\text {raw }}$ unknown $(H, K)$ in the set of 24 strongest.

Table 4.3. Coefficients (24 largest) of the Fourier transform of M17, with corresponding M16 PSF coefficient adjacent, showing resultant correction. Amplitudes are in arbitrary units (10000 max), phases in degrees.

\begin{tabular}{|r|r|r|r|r|r|r|r|}
\hline \multicolumn{2}{|l|}{$\begin{array}{l}\text { Coordinate (Fourier } \\
\text { space) }\end{array}$} & \multicolumn{2}{l|}{$I_{\text {raw_unknown }}(H, K)[\mathrm{M} 17]$} & \multicolumn{2}{l|}{$1 / \mathrm{P}_{\text {tip }}(H, K)[$ from M16] } & \multicolumn{2}{l|}{$I_{\text {corrected_unknown }}(H, K)$} \\
\hline$H$ & $K$ & amplitude & phase & amplitude & phase & amplitude & phase \\
\hline 0 & 1 & 10000 & -37 & 0.757 & 0 & 7570 & -37 \\
\hline 1 & 0 & 9522 & -89 & 0.822 & 0 & 7827 & -89 \\
\hline 1 & -1 & 3422 & -45 & 2.163 & -1 & 7402 & -46 \\
\hline 1 & 1 & 2214 & 88 & 0.546 & -13 & 1209 & 75 \\
\hline 0 & 2 & 1837 & 103 & 0.548 & 19 & 1007 & 122 \\
\hline 2 & 1 & 1341 & -67 & 0.489 & 10 & 656 & -57 \\
\hline 1 & 2 & 1055 & -15 & 0.408 & 19 & 430 & 4 \\
\hline 1 & 3 & 774 & 130 & 0.262 & 20 & 203 & 150 \\
\hline 2 & 0 & 727 & 56 & 1.232 & 91 & 896 & 147 \\
\hline 1 & -2 & 640 & -57 & 1.437 & -166 & 920 & -223 \\
\hline 2 & 2 & 599 & 170 & 0.402 & 4 & 241 & 174 \\
\hline 2 & -1 & 565 & 151 & 2.108 & -109 & 1191 & 42 \\
\hline 3 & 6 & 526 & 59 & 0.175 & 2 & 92 & 61 \\
\hline 0 & 3 & 521 & -65 & 0.388 & 9 & 202 & -56 \\
\hline 3 & -1 & 483 & -113 & 1.372 & 81 & 663 & -32 \\
\hline 2 & -2 & 412 & 152 & 2.751 & 140 & 1133 & 292 \\
\hline 4 & 8 & 337 & 177 & 0.199 & -1 & 67 & 176 \\
\hline 2 & 4 & 336 & -175 & 0.305 & -16 & 103 & -191 \\
\hline 3 & 1 & 323 & 100 & 0.919 & 33 & 297 & 133 \\
\hline 1 & 4 & 307 & -69 & 0.342 & 35 & 105 & -34 \\
\hline 2 & 3 & 290 & 16 & 0.365 & -47 & 106 & -31 \\
\hline 2 & 6 & 289 & -104 & 0.422 & -3 & 122 & -107 \\
\hline 3 & 2 & 264 & -4 & 0.549 & -64 & 145 & -68 \\
\hline 3 & 7 & 235 & -126 & $\# D I V / 0 !$ & $\# D I V / 0 !$ & $\# D I V / 0 !$ & $\# D I V / 0 !$ \\
\hline
\end{tabular}


We will produce a corrected image without any $k_{\text {bias }}$, that means there is one problematic coefficient $(3,7)$ for which the amplitude of M16's $I_{\text {observed }}(3,7)=0$ (the phase of $I_{\text {observed }}(3,7)$ is 5 degrees but that is irrelevant). We propose to disregard this coefficient in the first approximation, produce an image, then show what that image would look like with an increasing value of this coefficient; then we will produce an image with a positive $k_{\text {bias }}$.

Figure 4.9 shows the corrected M17 image using the coefficients in the right two columns of Table 4.3 above.

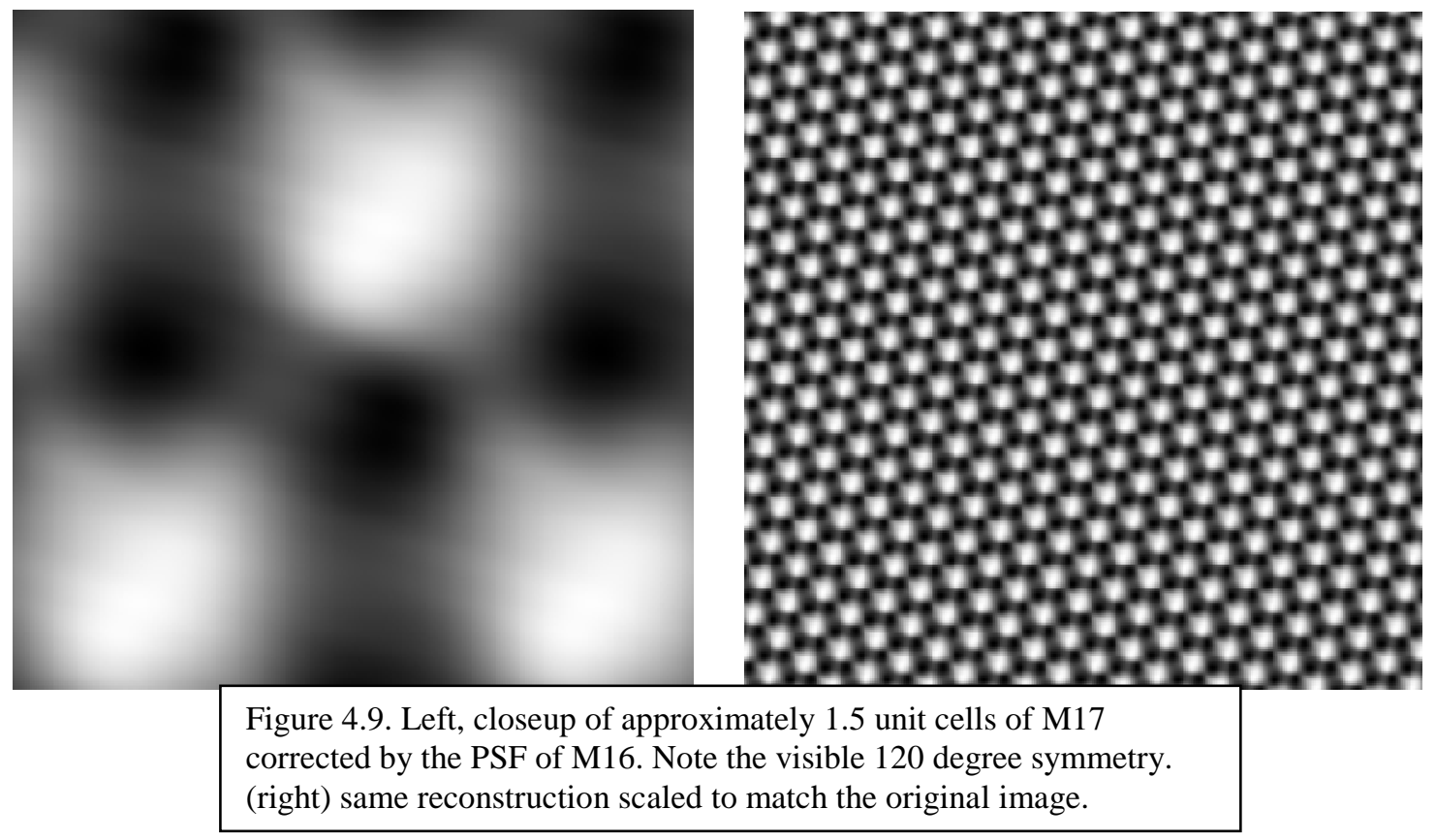

One can see the hexagonal lattice in the image. Processing this reconstructed image using CIP finds the actual angle between the reciprocal lattice axes to be 61.4 degrees, and calculation of residuals finds $p 3 m 1$ to be the best fit. See figure 4.10. 


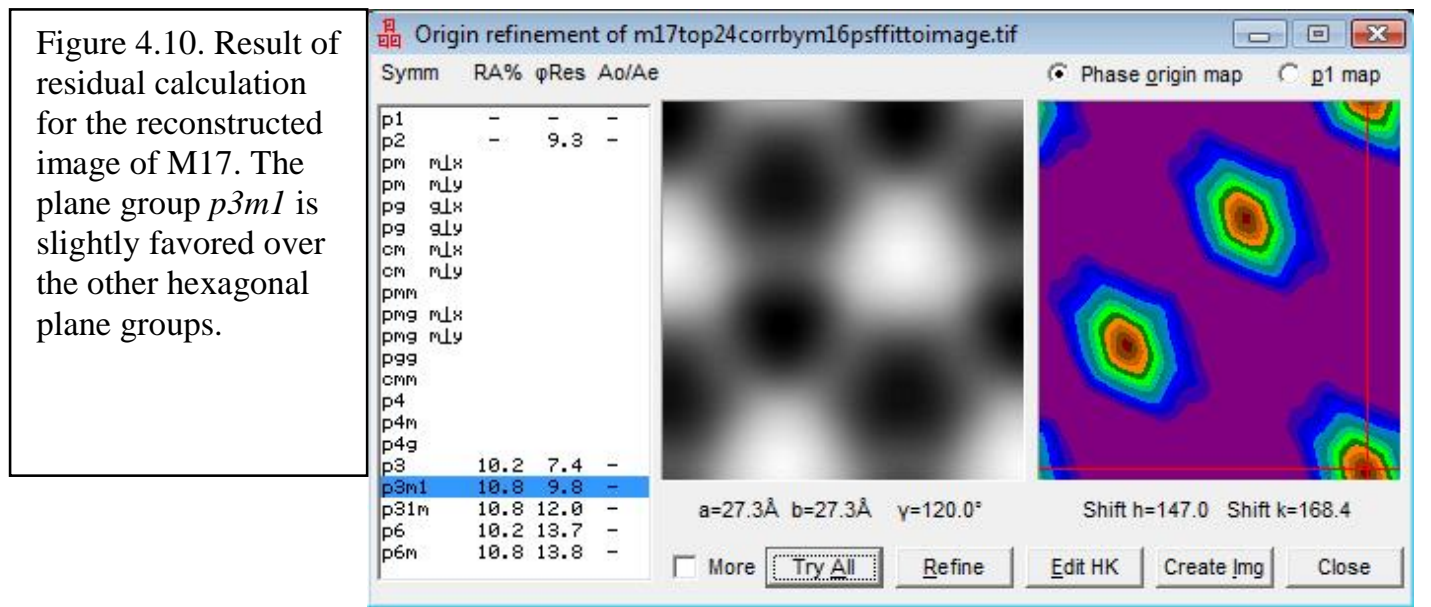

We see in the penultimate column of table 4.3 that the amplitudes of the corrected M17

coefficients range from a low of 67 to a high of 7827. These are arbitrary units, and matter only in relation to one another. Let us introduce the $(3,7)$ coefficient with arbitrary amplitudes of 400 and 4000. Since M16's $I_{\text {observed }}(3,7)=0$ the theoretical value of $I_{\text {corrected_unknown }}(3,7)$ is infinite. The three results are shown in Figure 4.11.

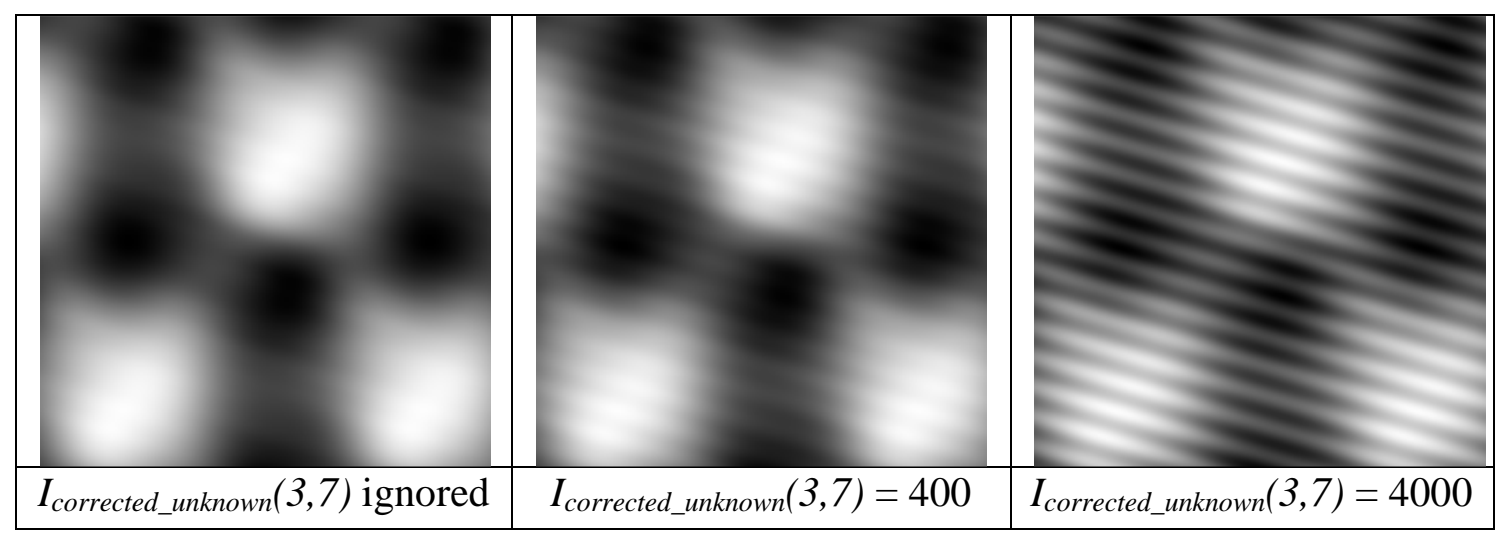

Figure 4.11. Three presentations of M17 corrected by the M16 PSF with various amplitude values for an anomalous coefficient. 
The anomalous $I_{\text {corrected_unknown }}(3,7)$ coefficient introduces a single wave into the image.

\subsubsection{Production of corrected M17 coefficients using a bias}

The values for amplitude of $P_{\text {tip }}$ range from 0 to approximately 5 (before the bias is applied - see table 4.2) for the range of $H, K$ coefficients we are considering here. If we arbitrarily institute a bias of +1 and add that bias to each $P_{\text {tip }}$ amplitude, and then recalculate $1 / P_{\text {tip }}$ and apply that to the M17 coefficients we obtain a new set of corrected coefficients.

Table 4.4. Coefficients (24 largest) of the Fourier transform of M17, with a biased M16 PSF coefficient adjacent, showing resultant correction. Amplitudes have dynamic range with 10000 max, phases in degrees.

\begin{tabular}{|r|r|r|r|r|r|r|r|}
\hline \multicolumn{2}{|l|}{$\begin{array}{l}\text { Coordinate (Fourier } \\
\text { space) }\end{array}$} & \multicolumn{2}{l}{$I_{\text {raw_unknown }}(H, K)[\mathrm{M} 17]$} & \multicolumn{2}{l|}{$1 /\left(\mathrm{P}_{\text {tip }}(H, K)+1+0 \mathrm{i}\right)$} & \multicolumn{2}{l|}{$I_{\text {corrected_unknown }}(H, K)$} \\
\hline$H$ & $K$ & amplitude & phase & amplitude & phase & amplitude & phase \\
\hline 0 & 1 & 10000 & -37 & 0.431 & 0 & 4308 & -37 \\
\hline 1 & 0 & 9522 & -89 & 0.451 & 0 & 4296 & -89 \\
\hline 1 & -1 & 3422 & -45 & 0.684 & -1 & 2340 & -46 \\
\hline 1 & 1 & 2214 & 88 & 0.353 & -13 & 782 & 75 \\
\hline 0 & 2 & 1837 & 103 & 0.354 & 19 & 650 & 122 \\
\hline 2 & 1 & 1341 & -67 & 0.328 & 10 & 440 & -57 \\
\hline 1 & 2 & 1055 & -15 & 0.290 & 19 & 306 & 4 \\
\hline 1 & 3 & 774 & 130 & 0.208 & 20 & 161 & 150 \\
\hline 2 & 0 & 727 & 56 & 0.552 & 91 & 401 & 147 \\
\hline 1 & -2 & 640 & -57 & 0.590 & -166 & 377 & -223 \\
\hline 2 & 2 & 599 & 170 & 0.287 & 4 & 172 & 174 \\
\hline 2 & -1 & 565 & 151 & 0.678 & -109 & 383 & 42 \\
\hline 3 & 6 & 526 & 59 & 0.149 & 2 & 78 & 61 \\
\hline 0 & 3 & 521 & -65 & 0.280 & 9 & 146 & -56 \\
\hline 3 & -1 & 483 & -113 & 0.578 & 81 & 279 & -32 \\
\hline 2 & -2 & 412 & 152 & 0.733 & 140 & 302 & 292 \\
\hline 4 & 8 & 337 & 177 & 0.166 & -1 & 56 & 176 \\
\hline 2 & 4 & 336 & -175 & 0.234 & -16 & 79 & -191 \\
\hline
\end{tabular}




\begin{tabular}{|r|r|r|r|r|r|r|r|}
\hline 3 & 1 & 323 & 100 & 0.479 & 33 & 155 & 133 \\
\hline 1 & 4 & 307 & -69 & 0.255 & 35 & 78 & -34 \\
\hline 2 & 3 & 290 & 16 & 0.268 & -47 & 78 & -31 \\
\hline 2 & 6 & 289 & -104 & 0.297 & -3 & 86 & -107 \\
\hline 3 & 2 & 264 & -4 & 0.354 & -64 & 94 & -68 \\
\hline 3 & 7 & 235 & -126 & 1.000 & 0 & 235 & -126 \\
\hline
\end{tabular}

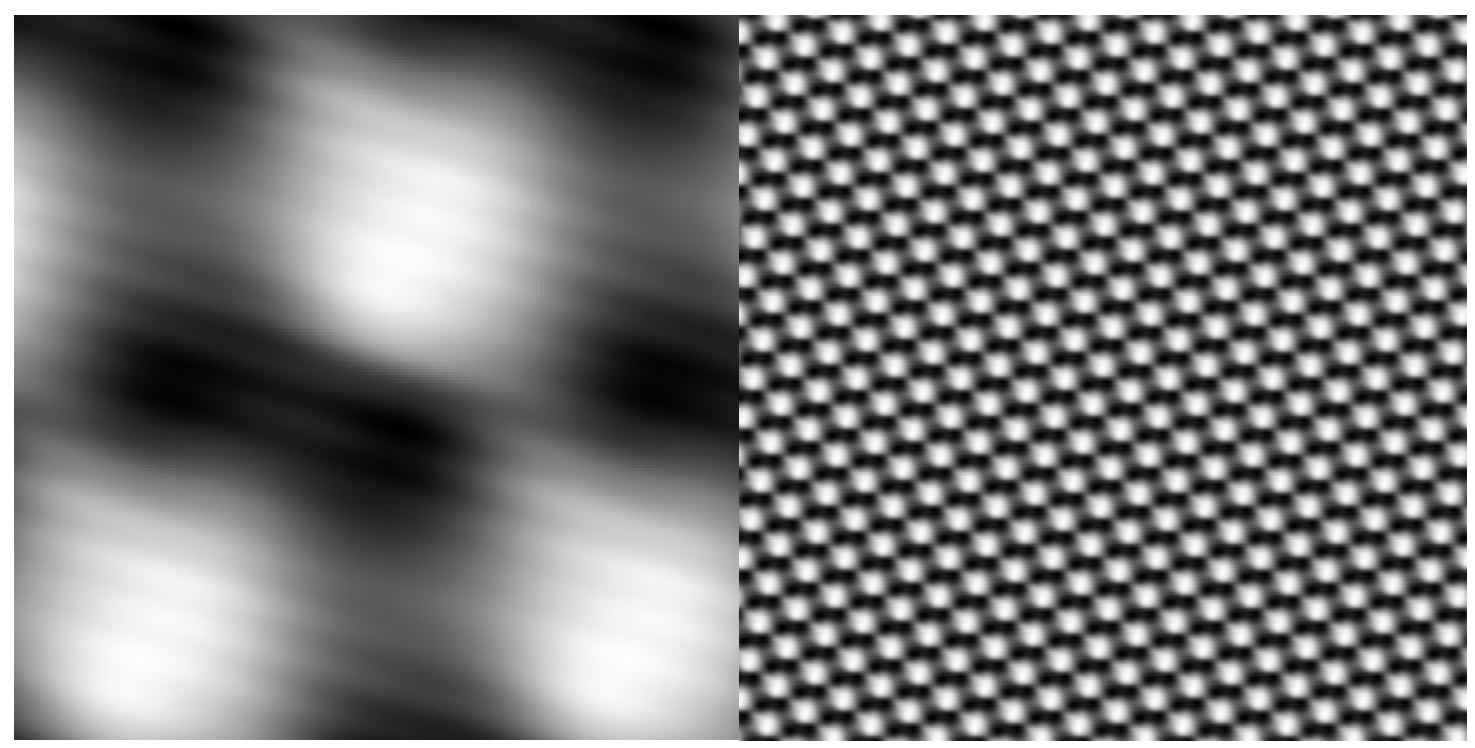

Figure 4.12. Left, closeup of approximately 1.5 unit cells of M17 corrected by the biased PSF of M16. Note that the hexagonal lattice is visibly less clear, see text for quantitative detail. (right) same reconstruction scaled to match the original image.

The image with this set of corrected coefficients is shown in figure 4.12. It is "muddier" than the image without the bias and there is a visible contribution from the $(3,7)$ component which is to be expected. Comparing this image to the raw image (figure 4.8 ) it seems to be that the $(3,7)$ component is a manifestation of the visible rastering in the raw image, in other words an artifact of the scanning process, and thus can be safely disregarded. 
This reconstructed image was also subjected to CIP and the residuals are notably higher, leading one to conclude that reconstruction without biasing out the zero value for this PSF coefficient is more effective. See table 4.5.

Table 4.5 Residuals for two different reconstructions of M17 using M16's PSF, first disregarding the zero PSF coefficient, then biasing it.

\begin{tabular}{|l|c|c|c|c|c|c|}
\hline & \multicolumn{2}{|c|}{$p 3$} & \multicolumn{2}{c|}{$p 3 m 1$} & \multicolumn{2}{c|}{$p 31 m$} \\
\cline { 2 - 7 } & RA\% & $\varphi$ Res & RA\% & $\varphi$ Res & RA\% & $\varphi$ Res \\
\hline $\begin{array}{l}\text { without bias to PSF } \\
\text { amplitudes (Fig. 4.9) }\end{array}$ & 10.2 & 7.4 & 10.8 & 9.8 & 10.8 & 12.0 \\
\hline $\begin{array}{l}\text { with +1 bias to PSF } \\
\text { amplitudes (Fig. 4.12) }\end{array}$ & 33.5 & 8.8 & 34.6 & 12.1 & 34.6 & 11.9 \\
\hline
\end{tabular}

By indexing the actual FFT plot, shown in figure 4.13, we see that the spot most closely associated with $(3,7)$ is visibly part of the Fourier noise generated by the horizontal striations in the image.

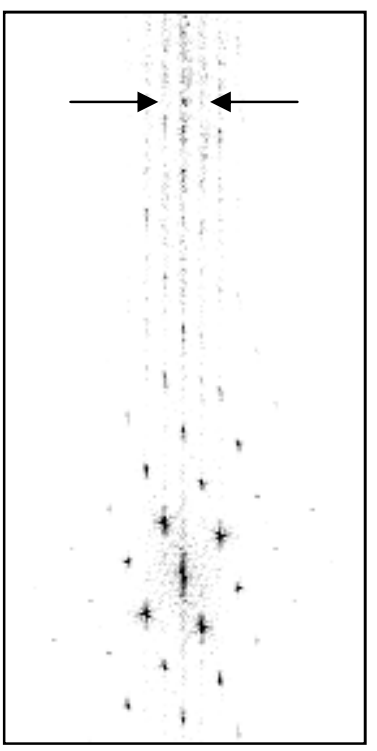

Figure 4.13. The arrows point to the $(3,7)$ spot in the FFT of M17. 


\subsection{Real space reconstruction of the effective tip PSF}

The fact that the point spread function $p_{t i p}(x, y)$ represents the entity in real space with which the object is convoluted in order to produce an image means that the point spread function is the actual morphology of the physical tip (as amended by other instrumental distortions).

The convolution of a periodic function with a nonperiodic impulse (in this case the tip) is periodic. We have a two-dimensional array $P_{t i p}(H, K)$ that is the Fourier transform of the PSF. Since our sample is periodic, we expect $P_{t i p}(H, K)$ to be periodic too. A density plot of one unit cell of $1 / P_{\text {tip }}(H, K)$, the inverse of the transform of the effective tip, is shown in figure 4.14

When we inverse Fourier transform $P_{t i p}$ the result, $p_{t i p}$, is a periodic function as well, the period of which should be equal to the longest repeating unit in the sample (provided we are including the lowest order coefficients $(0,1)$ and $(1,0)$ ). Thus the unit cell would be similarly sized to the sample molecule. The motif of this function should be the representation $p_{t i p}(x, y)$ of the tip that we seek. 


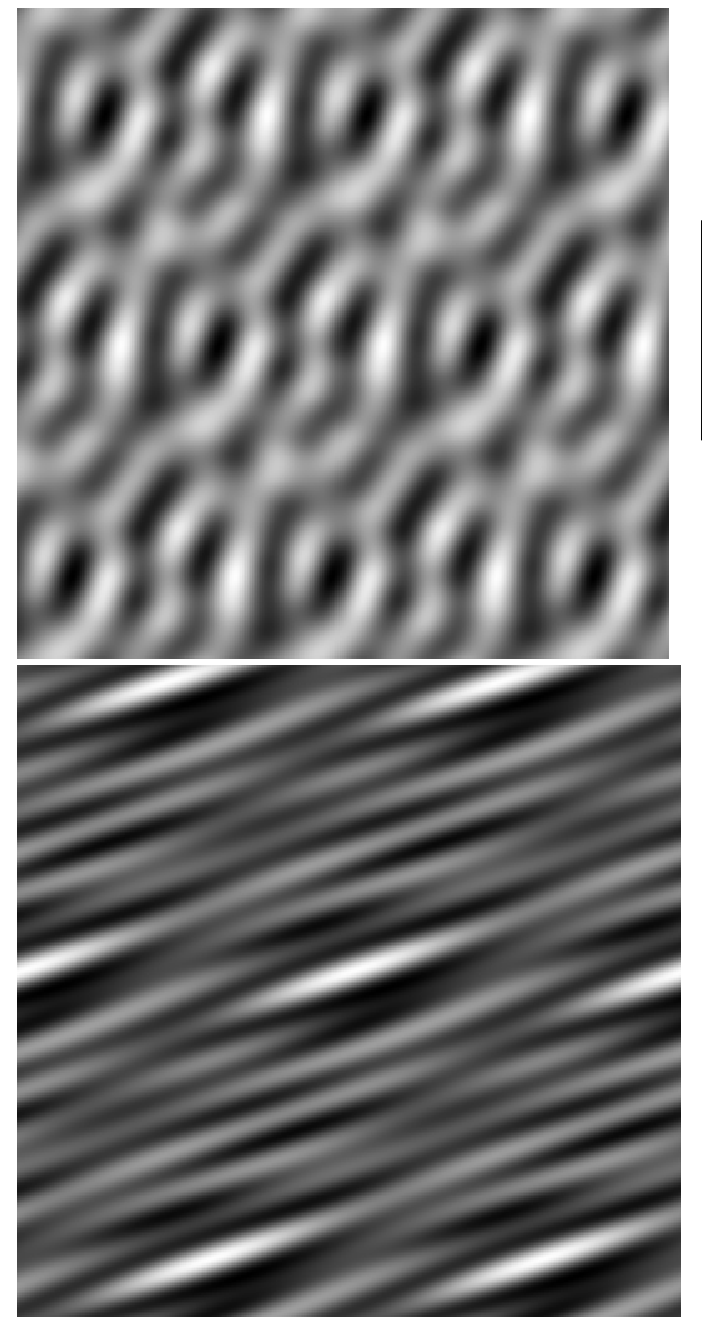

Figure 4.14.

Approximately 2.5 unit

cells of the inverse PSF of

the M16 effective tip.

Figure 4.15. Approximately two unit cells of the M16 PSF. (left) density plot; (right) contour plot. Length of arrow is approximately $1.5 \mathrm{~nm}$ as period of PSF is equal to that of M16.

When we perform this calculation for M16 the data is shown in table 4.6 and plotted in figure 4.15. Ten coefficients with maximum amplitude are shown (plotting more adds virtually no detail to the visual appearance), and several periodic motifs or approximately two units cells are plotted. The resulting elongated elliptical object is a representation in real space of the shape of the tip + instrument. If we assume that the 
periodicity in this image (shown by arrow) is equal to the unit cell dimension of M16, $1.5 \mathrm{~nm}$, we can estimate the dimension of the elliptical effective tip shown as approximately $1 \mathrm{~nm}$ long and $0.2 \mathrm{~nm}$ wide.

Table 4.6. 10 highest M16 PSF coefficients ranked by $P_{\text {tip }}$ amplitude, plus data for $(0,1)$ and $(1,0)$ coefficients.

\begin{tabular}{|c|c|c|c|c|c|c|c|}
\hline \multirow[b]{2}{*}{$H$} & \multirow[b]{2}{*}{$K$} & \multicolumn{2}{|c|}{ amplitude } & \multicolumn{2}{|c|}{ phase } & \multicolumn{2}{|c|}{$P_{t i p}(H, K)$} \\
\hline & & raw & symm & raw & symm & $\rho$ & $\theta$ \\
\hline 3 & 6 & 257 & 45 & -159 & -157 & 5711 & -2 \\
\hline 1 & 3 & 906 & 237 & -175 & -155 & 3823 & -20 \\
\hline 2 & 4 & 308 & 94 & -21 & -37 & 3277 & 16 \\
\hline 1 & 4 & 281 & 96 & 11 & 46 & 2927 & -35 \\
\hline 2 & 3 & 353 & 129 & 76 & 29 & 2736 & 47 \\
\hline 0 & 3 & 500 & 194 & -116 & -107 & 2577 & -9 \\
\hline 2 & 2 & 592 & 238 & 176 & 180 & 2487 & -4 \\
\hline 1 & 2 & 1374 & 561 & 87 & 106 & 2449 & -19 \\
\hline 2 & 6 & 45 & 19 & 55 & 52 & 2368 & 3 \\
\hline 2 & 1 & 1147 & 561 & -116 & -106 & 2045 & -10 \\
\hline 0 & 1 & 10000 & 7565 & -10 & -10 & 1322 & 0 \\
\hline 1 & 0 & 9198 & 7565 & 10 & 10 & 1216 & 0 \\
\hline
\end{tabular}

This elongated elliptical image is dominated by several high order Fourier coefficients that are relatively unimportant in the transform of the original sample. Keeping in mind that each individual $P_{\text {tip }}$ coefficient is calculated by $I_{\text {raw }} / I_{\text {symmetrized }}$ at a particular $\mathrm{H}, \mathrm{K}$ value, it is interesting that these prominent high-order coefficients have high amplitudes not because $I_{\text {raw }}$ is high (quite the opposite), but because $I_{\text {symmetrized }}$ is very low.

For example, for the high order $(H, K)=(3,6)$, the amplitude of $I_{\text {raw }}$ is only 257 (arbitrary units) but the amplitude of $I_{\text {symmetrized }}$ is much lower, at 45 , thus the amplitude of $P_{\text {tip }}(3,6)$ is very high, 5711 (normalized, arbitrary units). Compare that with the 
coefficient $(H, K)=(0,1)$, which is a much stronger coefficient in the original image with an amplitude of 10000 ; but $I_{\text {symmetrized }}(0,1)$ also has a high amplitude at 7565 , therefore amplitude of $P_{t i p}(0,1)=1322$, about $1 / 4$ as strong as that of $P_{t i p}(3,6)$.

If we weight the $P_{t i p}$ coefficients according to their original amplitude strength in the raw image, we obtain a different set of 10 maximum amplitude coefficients, whose image is seen in figure 4.16. The length of this object is the same as the unweighted version in figure 4.15 , but the width is considerably greater.

Figure 4.17 shows close-ups of both unweighted and weighted periodic motifs for the M16 PSF.
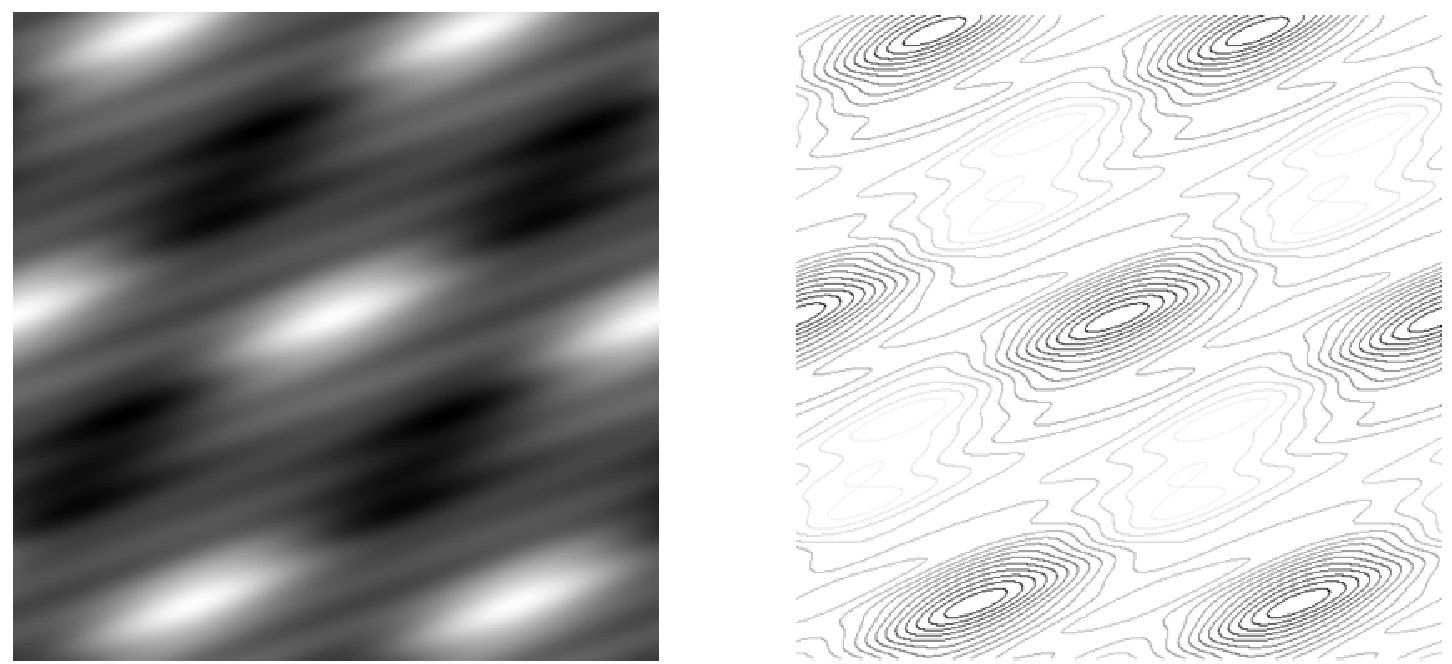

Figure 4.16. Approximately two unit cells of the M16 PSF after weighting coefficients as described in text. (left) density plot; (right) contour plot. 

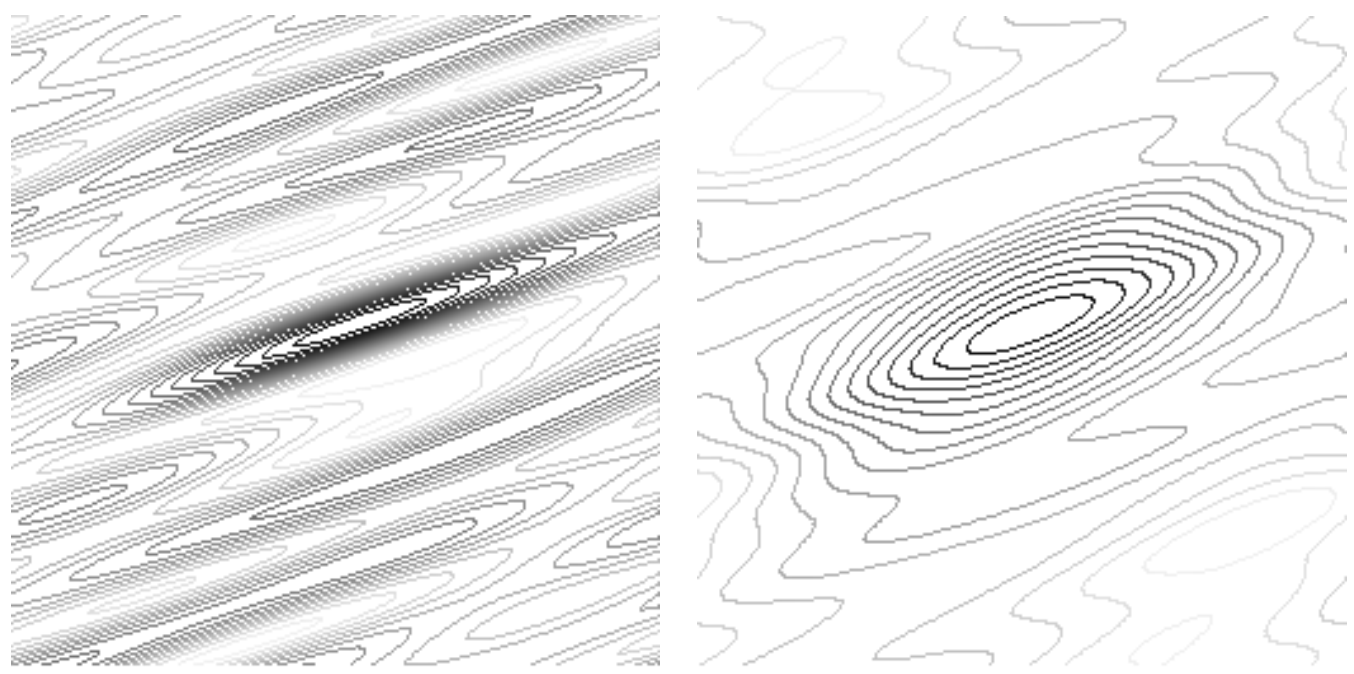

Figure 4.17. Approximately one periodic motif of M16 PSF, contour plot.(left) unweighted; (right) weighted.

Table 4.7. Ten highest M16 coefficients ranked by $P_{\text {tip }}$ amplitude after weighting by $I_{\text {raw }}$ amplitude.

\begin{tabular}{|c|c|c|c|c|c|c|c|}
\hline \multirow[b]{2}{*}{$H$} & \multirow[b]{2}{*}{$K$} & \multicolumn{2}{|c|}{ amplitude } & \multicolumn{2}{|c|}{ phase } & \multicolumn{2}{|c|}{$P_{\text {tip }}(H, K)$} \\
\hline & & raw & symm & raw & symm & amplitude & phase \\
\hline 0 & 1 & 10000 & 7565 & -10 & -10 & 6609 & 0 \\
\hline 1 & 0 & 9198 & 7565 & 10 & 10 & 5592 & 0 \\
\hline 1 & 1 & 2072 & 1132 & -167 & 180 & 1896 & 13 \\
\hline 1 & 3 & 906 & 237 & -175 & -155 & 1732 & -20 \\
\hline 1 & 2 & 1374 & 561 & 87 & 106 & 1683 & -19 \\
\hline 0 & 2 & 1550 & 850 & 38 & 57 & 1413 & -19 \\
\hline 2 & 1 & 1147 & 561 & -116 & -106 & 1173 & -10 \\
\hline 1 & -1 & 3497 & 7565 & -9 & -10 & 808 & 1 \\
\hline 2 & 2 & 592 & 238 & 176 & 180 & 736 & -4 \\
\hline 3 & 6 & 257 & 45 & -159 & -157 & 734 & -2 \\
\hline
\end{tabular}

Weighting the coefficients is an empirical procedure, not theoretical, that appears to change the model into a more reasonable representation of a tip. Further work is required to validate this model. 


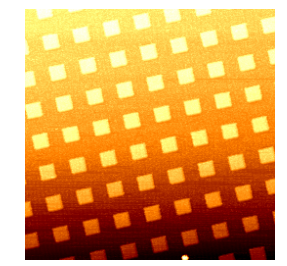

Figure 3.34

We have tried the same technique on a different image, figure 3.35, which is the open-loop AFM image of a square calibration grid (also shown in figure 3.34 as a closed-loop image). In this case $I_{o b s} / I_{\text {sym }}$ yielded 39 usable coordinates for $P_{t i p}$, many of them of high order,

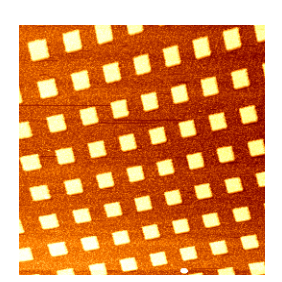
probably because of the sharp edges of the sample. The enforced symmetry was $p 4 m m$. The coordinates are shown in table 4.8 and the PSF is plotted in figure 4.18. It appears as a narrow tip with slight

Figure 3.35 asymmetry around the tip (visible in the contour plot), which is consistent with a well-operating instrument with a good tip that has long-period hysteresis over the entire image. For comparison figure 4.19 shows a similar plot of the PSF of the closed-loop image of the same sample, figure 3.34, and table 4.9 shows its coefficients. The reconstructed tip appears sharper as one might expect with no hysteresis in the image. In both cases, closed- and open-loop, the dimension of the unit cell of the PSF (highlighted with arrows) is equal to the periodicity of the sample, here $10 \mu \mathrm{m}$. 


\begin{tabular}{|c|c|c|c|c|c|c|c|}
\hline \multicolumn{8}{|c|}{ Table 4.8 PSF calculation for figure 3.35 (open loop) } \\
\hline \multirow[b]{2}{*}{$H$} & \multirow[b]{2}{*}{$K$} & \multicolumn{2}{|c|}{ amplitude } & \multicolumn{2}{|c|}{ phase } & \multicolumn{2}{|c|}{$P_{\text {tip }}[$ FT of PSF] } \\
\hline & & obs & symm & obs & symm & $\rho$ & $\theta$ \\
\hline 1 & 2 & 803 & 322 & -50 & 0 & 2494 & 10 \\
\hline 0 & 4 & 570 & 285 & 153 & 180 & 2000 & 3 \\
\hline 1 & -4 & 504 & 268 & -160 & 180 & 1881 & 20 \\
\hline 7 & 1 & 635 & 365 & -157 & 180 & 1740 & 19 \\
\hline 5 & 1 & 1115 & 708 & -15 & 0 & 1575 & 17 \\
\hline 1 & -2 & 485 & 322 & -4 & 0 & 1506 & -4 \\
\hline 0 & 6 & 433 & 300 & 10 & 0 & 1443 & 5 \\
\hline 0 & 2 & 1075 & 745 & -37 & 0 & 1443 & 1 \\
\hline 5 & 0 & 1403 & 1029 & 36 & 0 & 1363 & 36 \\
\hline 3 & 1 & 1986 & 1568 & 167 & 180 & 1267 & 13 \\
\hline 1 & 4 & 338 & 268 & 161 & 180 & 1261 & 14 \\
\hline 9 & 0 & 367 & 301 & 35 & 0 & 1219 & 35 \\
\hline 0 & 8 & 255 & 211 & -153 & 180 & 1209 & 7 \\
\hline 7 & 0 & 714 & 597 & -173 & 180 & 1196 & 7 \\
\hline 3 & 0 & 2877 & 2414 & 178 & 180 & 1192 & -2 \\
\hline 3 & -1 & 1796 & 1568 & -130 & 180 & 1145 & 50 \\
\hline 3 & 3 & 390 & 363 & -38 & 0 & 1074 & 20 \\
\hline 5 & -1 & 745 & 708 & 15 & 0 & 1052 & 15 \\
\hline 1 & 1 & 6926 & 6767 & -26 & 0 & 1023 & 9 \\
\hline 0 & 1 & 10000 & 9942 & -20 & 0 & 1006 & 0 \\
\hline 1 & 0 & 9884 & 9942 & 4 & 0 & 994 & 4 \\
\hline 1 & -1 & 6609 & 6767 & 12 & 0 & 977 & 12 \\
\hline 3 & -3 & 337 & 363 & -22 & 0 & 928 & -22 \\
\hline 1 & -7 & 334 & 365 & 154 & 180 & 915 & -26 \\
\hline 7 & -1 & 313 & 365 & -133 & 180 & 858 & 47 \\
\hline 1 & 3 & 1324 & 1568 & 173 & 180 & 844 & 12 \\
\hline 0 & 3 & 1951 & 2414 & 160 & 180 & 808 & 2 \\
\hline 0 & 7 & 481 & 597 & -158 & 180 & 806 & 6 \\
\hline 8 & 0 & 167 & 211 & 110 & 180 & 791 & -70 \\
\hline 0 & 9 & 235 & 301 & 14 & 0 & 781 & 8 \\
\hline 1 & -3 & 1164 & 1568 & -168 & 180 & 742 & 12 \\
\hline 4 & -1 & 193 & 268 & 145 & 180 & 720 & -35 \\
\hline 1 & -5 & 487 & 708 & 4 & 0 & 688 & 4 \\
\hline 1 & 5 & 486 & 708 & 11 & 0 & 686 & 16 \\
\hline 0 & 5 & 656 & 1029 & -13 & 0 & 638 & 4 \\
\hline 2 & 0 & 416 & 745 & -58 & 0 & 558 & -58 \\
\hline 6 & 0 & 167 & 300 & -17 & 0 & 557 & -17 \\
\hline 1 & 7 & 177 & 365 & 159 & 180 & 485 & 18 \\
\hline 4 & 1 & 38 & 268 & -133 & 180 & 142 & 15 \\
\hline
\end{tabular}




\begin{tabular}{|c|c|c|c|c|c|c|c|}
\hline \multicolumn{8}{|c|}{ Table 4.9 PSF calculation for figure 3.34 (closed loop) } \\
\hline \multirow[b]{2}{*}{$H$} & \multirow[b]{2}{*}{$K$} & \multicolumn{2}{|c|}{ amplitude } & \multicolumn{2}{|c|}{ phase } & \multicolumn{2}{|c|}{$P_{\text {tip }}[$ FT of PSF] } \\
\hline & & obs & symm & obs & symm & $\rho$ & $\theta$ \\
\hline 8 & 1 & 450 & 245 & 10 & 0 & 1837 & 10 \\
\hline 10 & 0 & 491 & 291 & -5 & 0 & 1687 & -5 \\
\hline 8 & -1 & 398 & 245 & -5 & 0 & 1624 & -5 \\
\hline 8 & 0 & 799 & 496 & -179 & 180 & 1611 & 1 \\
\hline 2 & 1 & 913 & 573 & -177 & 180 & 1593 & 3 \\
\hline 2 & 0 & 1859 & 1200 & -29 & 0 & 1549 & -29 \\
\hline 4 & -1 & 936 & 606 & 6 & 0 & 1545 & 6 \\
\hline 6 & -1 & 720 & 468 & 176 & 180 & 1538 & -4 \\
\hline 6 & 0 & 1071 & 722 & -15 & 0 & 1483 & -15 \\
\hline 4 & 0 & 1352 & 936 & -179 & 180 & 1444 & 1 \\
\hline 6 & 1 & 663 & 468 & -167 & 180 & 1417 & 13 \\
\hline 0 & 11 & 320 & 231 & 36 & 0 & 1385 & 36 \\
\hline 4 & 1 & 759 & 606 & -1 & 0 & 1252 & -1 \\
\hline 0 & 9 & 526 & 423 & -166 & 180 & 1243 & 14 \\
\hline 2 & -1 & 699 & 573 & -162 & 180 & 1220 & 18 \\
\hline 1 & 9 & 384 & 316 & 20 & 0 & 1215 & 20 \\
\hline 1 & -9 & 368 & 316 & -34 & 0 & 1165 & -34 \\
\hline 1 & -7 & 628 & 556 & 167 & 180 & 1129 & -13 \\
\hline 1 & 5 & 1144 & 1014 & 1 & 0 & 1128 & 1 \\
\hline 3 & -5 & 337 & 302 & 179 & 180 & 1116 & -1 \\
\hline 5 & 3 & 323 & 302 & 176 & 180 & 1070 & -4 \\
\hline 1 & 7 & 588 & 556 & 175 & 180 & 1058 & -5 \\
\hline 3 & 3 & 580 & 549 & 18 & 0 & 1056 & 18 \\
\hline 0 & 7 & 880 & 838 & 15 & 0 & 1050 & 15 \\
\hline 3 & 1 & 2109 & 2019 & 172 & 180 & 1045 & -8 \\
\hline 1 & 3 & 2102 & 2019 & 169 & 180 & 1041 & -11 \\
\hline 3 & 0 & 3244 & 3121 & -8 & 0 & 1039 & -8 \\
\hline 1 & -3 & 2071 & 2019 & -179 & 180 & 1026 & 1 \\
\hline 0 & 13 & 204 & 201 & -159 & 180 & 1015 & 21 \\
\hline 0 & 5 & 1537 & 1517 & 179 & 180 & 1013 & -1 \\
\hline 1 & 0 & 10000 & 9925 & 177 & 180 & 1008 & -3 \\
\hline 1 & -5 & 1020 & 1014 & -13 & 0 & 1006 & -13 \\
\hline 1 & -1 & 6894 & 6878 & -12 & 0 & 1002 & -12 \\
\hline 1 & 1 & 6861 & 6878 & 0 & 0 & 998 & 0 \\
\hline 0 & 1 & 9849 & 9925 & 175 & 180 & 992 & -5 \\
\hline 5 & 0 & 1497 & 1517 & 180 & 180 & 987 & 0 \\
\hline 8 & 1 & 450 & 245 & 10 & 0 & 1837 & 10 \\
\hline 10 & 0 & 491 & 291 & -5 & 0 & 1687 & -5 \\
\hline 8 & -1 & 398 & 245 & -5 & 0 & 1624 & -5 \\
\hline
\end{tabular}




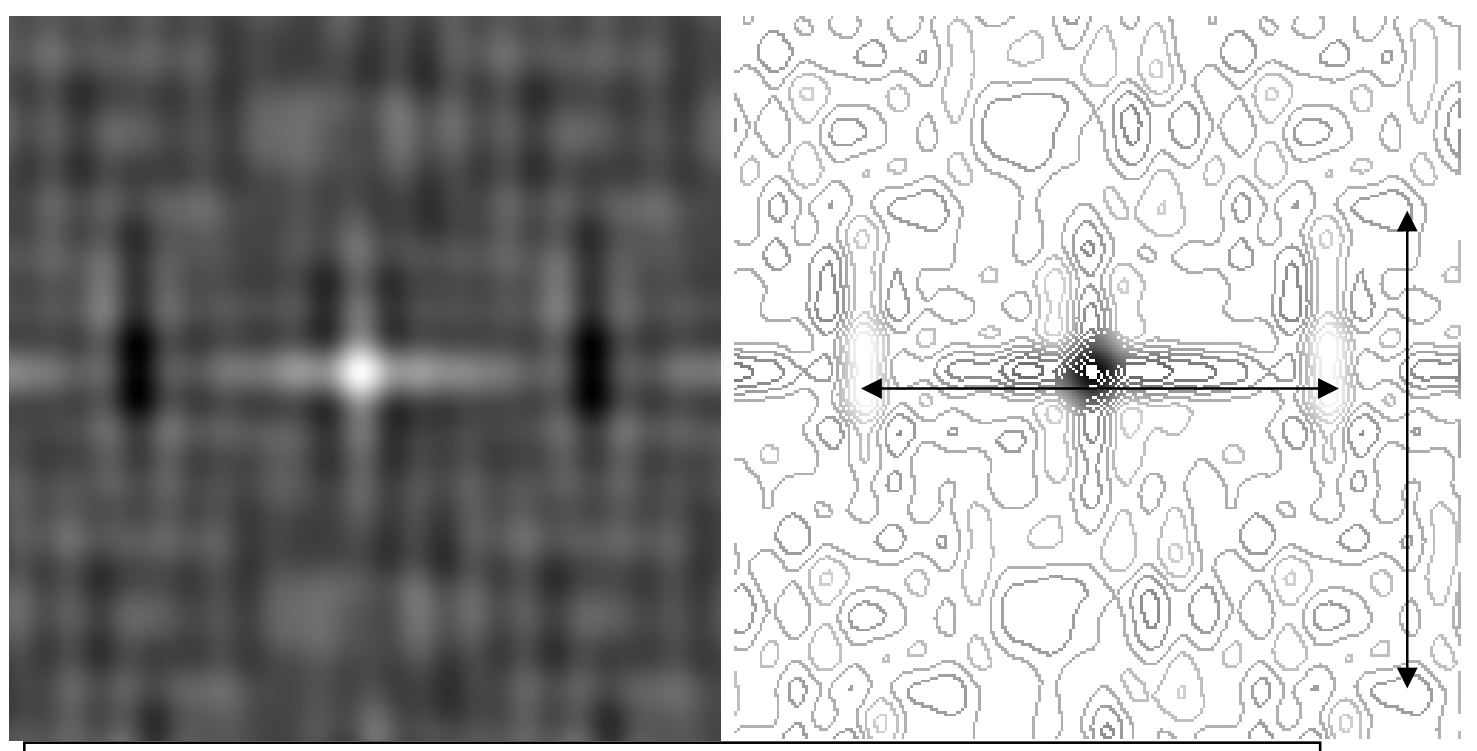

Figure 4.18. (left) Periodic motif density plot of PSF of open-loop figure 3.35; (right) Contour plot with length of one unit cell $(10 \mu \mathrm{m})$ highlighted
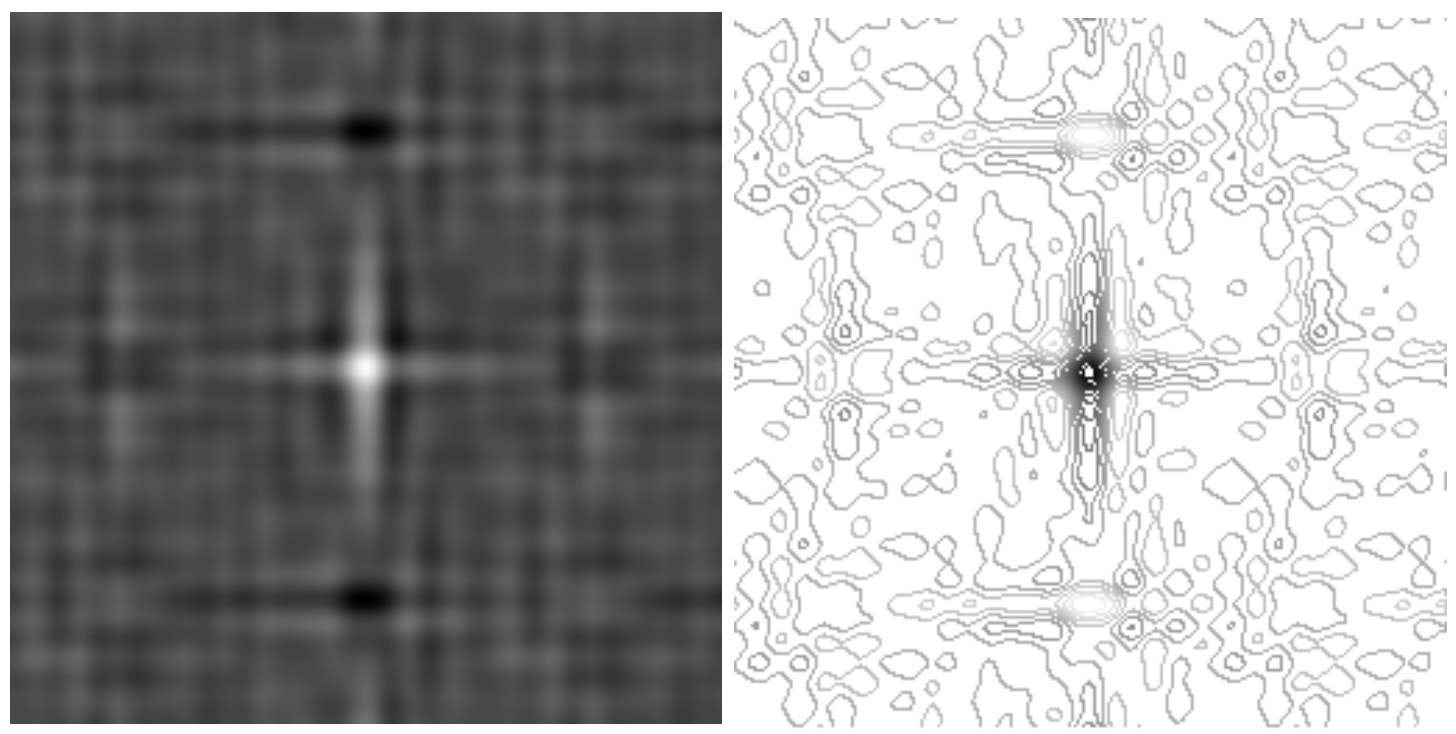

Figure 4.19. (left) Density plot of the periodic motif of the PSF of figure 3.34, closed-loop image (right) contour plot 


\section{Summary and Conclusion}

In this thesis we have shown that the technique of Crystallographic Image Processing, which was designed for the processing of high resolution (lattice fringe) images from TEMs, is also applicable to two-dimensional images of periodic samples taken by scanning probe microscopes. We demonstrated in this thesis that the use of the crystallographic averaging algorithm removes noise and random error from images more effectively than translational averaging. This was done by testing both CIP and translational averaging on simulated $512 \times 512$ pixel images with deliberately introduced random error, with motifs arranged in a $4 \times 4$ array and a $6 \times 6$ array. The results confirmed the effectiveness of CIP and the relationship between signal-to-noise ratio and sample size. We also created a $2048 \times 2048$ pixel simulated image with systematic error and showed the superior performance of CIP over translational averaging.

We then applied the technique to an STM image of $\mathrm{F}_{16} \mathrm{CoPc}$ on HOPG which appeared to have been taken with a blunt, multiple or otherwise imperfect tip. By selecting the only plane symmetry group $(p 4 m m)$ compatible with the known point symmetry of the molecule $(4 \mathrm{~mm})$ we were able to produce an image similar to other images of this molecule taken with tips lacking this type of defect. We also demonstrated the ability to correct systematic errors caused by hysteresis in the AFM image of a calibration grid, by imaging the grid with and without the instrument's closed-loop hysteresis correction operating. CIP was able to remove the visible nonlinearities in the image that had no hysteresis correction. This was effective even though 
the amplitude residual for the non-linear image was $20.6 \%$ vs. $16.8 \%$ and the phase residual was $20.7^{\circ}$ compared to $13.5^{\circ}$.

Given a scanning probe image of a 2D periodic, highly symmetric known sample, we demonstrated that it was possible to use the Fourier coefficients of the image transform to solve the inverse problem and calculate the point spread function (PSF) of the instrument. This was done by crystallographically enforcing a suitable plane symmetry group on an STM image of $\mathrm{F}_{16} \mathrm{CoPc}$ on $\mathrm{HOPG}$ and dividing the Fourier transform of the original image (as it was output by the instrument) by the transform of the enforced image. The quotient is the Fourier transform of the instrumental PSF. Its inverse Fourier transform, when plotted in real space, represents the net effect that the microscope itself has on the object-to-image transaction. The plot of the ten highestamplitude Fourier coefficients shown in the thesis depicts an elongated "tip" with a possible "minitip" adjacent. (It was found that plotting more than the 10 highestamplitude Fourier coefficients adds virtually no visual detail.)

We performed the same procedure on the two AFM images of a calibration grid, with and without hysteresis correction, and show that the real-space plot of the effective tip resembles a two-dimensional projection along the tip axis of a three-dimensional sharp tip, and is much sharper in the image which has hysteresis correction, supporting the conclusion that this real-space plot is representative of the actual instrument.

We additionally demonstrated that the PSF, once known, could be used on a second image taken by the same instrument under essentially the same experimental 
conditions to remove errors introduced during that second imaging process. This involves operating in Fourier space to divide out the contribution of the instrument. This was done with another STM image of $\mathrm{F}_{16} \mathrm{CoPc}$ which was taken under essentially the same experimental conditions, but is of a different area of the sample. Both this image, and the one from which the PSF was derived had vacancies and visible jitter. (This particular image also had a vertical discontinuity where a scanning parameter visibly changed.) After performing the PSF correction, we applied CIP to the "before" image and "after" image, and were able to show significantly improved residuals after the PSF correction; the phase residual for the corrected image was $9.8^{\circ}$ compared with $15.8^{\circ}$ before correction, and the amplitude residual was improved from $55.2 \%$ to $10.8 \%$. This process is mathematically sound when applied to a second image with plane group symmetry the same as the calibration image; further work is to be done to justify using this process on all types of images.

Further work is also in progress (which will apply to all uses of CIP for SPM images) to obtain a fully objective criterion, given a particular image, for choosing the plane symmetry group which should be enforced. 


\section{Publications/Presentations by author}

B. Moon, J. Straton, T. Bilyeu, P. Moeck, "Crystallographic image processing of twodimensionally periodic scanning probe microscope images", presented at Oregon Academy of Sciences 2011 meeting, Portland Community College, February 26, 2011

B. Moon, P. Plachinda, J. Straton, P. Moeck, "Crystallographic Image Processing for Atomic Force and Scanning Tunneling Microscopists", poster presentation at American Physical Society 2010 March meeting, Portland, March 16, 2010

P. Plachinda, B. Moon, P. Moeck, "Crystallographic Image Processing Software for Scanning Probe Microscopists", presentation at American Physical Society 2010 March meeting, Portland, March 17, 2010

B. Moon, P. Moeck, "Application of Crystallographic Image Processing (CIP) for Scanning Probe Microscopy Images", presentation at Oregon Academy of Sciences 2010 meeting, Concordia University, Portland, February 27, 2010

J. Straton, B. Moon, P. Moeck, "Clarifying Multiple-Tip Effects on Scanning Tunneling Microscopy Imaging and Crystallographic Filtering in the Spatial Frequency Domain", poster presented at Oregon Academy of Sciences 2010 meeting, Concordia University, Portland, February 27, 2010

P. Moeck, B. Moon, M. Abdel-Hafiez, M. Hietschold, "Quantifying and enforcing the two-dimensional symmetry of scanning probe microscopy images of periodic objects", Nanotechnology 2009: Fabrication, Particles, Characterization, MEMS, Electronics and Photonics, pp. 314-317

B. Moon, S. Rouvimov, P. Moeck, "Identification of nanocrystals through analysis of HRTEM lattice fringes", presentation at Oregon Academy of Sciences 2009 meeting, Western Oregon University, February 28, 2009 


\section{References}

1. Villarrubia, J.S., Algorithms for Scanned Probe Microscope Image Simulation, Surface Reconstruction, and Tip Estimation. Journal of Research of the National Institute of Standards and Technology, 1997. 102.

2. Dorset, D.L., Structural Electron Crystallography. 1995, New York: Plenum Press.

3. Klug, A., From Macromolecules to Biological assemblies. 1982, Chemistry: obel Lecture.http://nobelprize.org/nobel prizes/chemistry/laureates/1982/kluglecture.html

4. International Tables for Crystallography. $5^{\text {th }}$ ed. Space-group symmetry (brief teaching edition), ed. T. Hahn. Vol. A. 2005, Dordrecht: Springer.

5. Bjorge, R., Lattice-Fringe Fingerprinting: Structural identification of nanocrystals employing high-resolution transmission electron microscopy, in Physics. 2007, Portland State University. http://www.scientificjournals.org/journals2007/articles/1235.pdf

6. Zou, X., et al., Structure projection retrieval by image processing of HREM images taken under non-optimum defocus conditions. Ultramicroscopy, 1996. 62.

7. Kanatani, K., Comments on "Symmetry as a Continuous Feature". IEEE Transactions on Pattern Analysis and Machine Intelligence, 1997. 19: p. 246.

8. Zabrodsky, H., S. Peleg, and D. Avnir, Symmetry as a continuous feature. IEEE Transactions on Pattern Analysis and Machine Intelligence, 1995. 17: p. 11541166.

9. van Dyck, D., et al., High-resolution electron microscopy, in Advances in Imaging and Electron Physics. 2002, Elsevier. p. 105-171.

10. Oku, T., Direct observation of B84 and B156 clusters by high-resolution electron microscopy and crystallographic image processing. Solid State Communications, 2003. 127(9-10): p. 689-693.

11. Park, S. and C.F. Quate, Digital Filtering of scanning tunneling microscope images. J. Appl. Phys., 1987. 62: p. 312-314.

12. Gross, L., et al., The Chemical Structure of a Molecule Resolved by Atomic Force Microscopy. Science, 2009. 325(5944): p. 1110-1114.

13. Chen, C.J., Introduction to Scanning Tunneling Microscopy. Oxford Series in Optical and Imaging Sciences. 1993: Oxford University Press.

14. Giessibl, F.J., Advances in atomic force microscopy. Reviews of Modern Physics, 2003. 75: p. 949-978.

15. Binnig, G. and C.F. Quate, Atomic Force Microscope. Physical Review Letters, 1986. 56: p. 930-933.

16. Gan, Y., Atomic and subnanometer resolution in ambient conditions by atomic force microscopy. Surface Science Reports, 2009. 64(3): p. 99-121.

17. Howland, R. and L. Benatar, A Practical Guide to Scanning Probe Microscopy. 2000: Park Scientific. 78. 
18. Dongmo, L.S., et al., Experimental test of blind tip reconstruction for scanning probe microscopy. Ultramicroscopy, 2000. 85: p. 141-153.

19. Snyder, E.J., E.A. Eklund, and R.S. Williams, Effects of tip size and asymmetry on scanning tunneling microscope topographs. Surface Science, 1990. 239: p. L487-L492.

20. Changhai, R. and S. Lining, Hysteresis and creep compensation for piezoelectric actuator in open-loop operation. Sensors and Actuators A: Physical, 2005. 122: p. 124-130.

21. Toader, M., et al., Exploring the F16CoPc/Ag(110) Interface Using Scanning Tunneling Microscopy and Spectroscopy. Part 1: Template-Guided Adlayer Structure Formation. The Journal of Physical Chemistry C. 114(8): p. 35373543 .

22. Oh, Y., et al., $N$-type organic field-effect transistor using polymeric blend gate insulator with controlled surface properties. Organic Electronics, 2006. 7: p. 77-84.

23. Kataoka, T., et al., Observation of a temperature-dependent transition of a copper-phthalocyanine thin film adsorbed on HOPG. Chemical Physics Letters, 2008. 451: p. 43-47.

24. Moeck, P., Crystallographic image processing for scanning probe microscopy, in Microscopy: Science, Technology, Applications and Education A. MéndezVilas and J. Díaz, Editors. 2010, Formatex: Badajoz. p. 1951-1962. http://www.formatex.info/microscopy4/1951-1962.pdf

25. Hembacher, S., et al., Revealing the hidden atom in graphite by lowtemperature atomic force microscopy. Proceedings of the National Academy of Sciences of the United States of America, 2003. 100: p. 12539-42.

26. CRISP, Calidris, Inc., Manhemsvägen 4, SE-191 45 Sollentuna, Sweden. http://www.calidris-em.com/crisp.php

27. Hipps, K.W., et al., Metal d-Orbital Occupation-Dependent Images in the Scanning Tunneling Microscopy of Metal Phthalocyanines. The Journal of Physical Chemistry, 1996. 100(27): p. 11207-11210.

28. Ogunrinde, A., K.W. Hipps, and L. Scudiero, A Scanning Tunneling Microscopy Study of Self-Assembled Nickel(II) Octaethylporphyrin Deposited from Solutions on HOPG. Langmuir, 2006. 22(13): p. 5697-5701.

29. Duncan, D. 2010, Oregon Health \& Science University: Portland, OR. 


\section{Appendix A - Source code for simulated images}

Code used to generate the simulated images of crosses in Section 3. Code written in Visual Basic 2008 Express Edition.

\section{Public Class Crosses}

Private Sub PictureBox1_Click(ByVal sender As System.Object, ByVal e As

System.EventArgs) Handles PictureBox1.Click

Dim imagesize As Integer

imagesize = CInt(InputBox("Pixel size of image (power of 2)?"))

Dim im As New Bitmap(imagesize, imagesize)

Dim b As Graphics = Graphics.FromImage $(i m)$

Dim crossl, crossw As Integer

Dim x, y As Integer

Dim DistortCross As MsgBoxResult

Dim BiasCross As MsgBoxResult

' Dim BiasAmount As Integer

crossl $=$ CInt $($ InputBox("Pixel length of cross, multiple of 10?"))

crossw $=$ crossl $\backslash 5$

DistortCross = MsgBox("Do you want to distort the crosses?", MsgBoxStyle.YesNo)

Randomize 0

If Not DistortCross Then

BiasCross = MsgBox("Do you want to bias the crosses?", MsgBoxStyle.YesNo)

End If

'If BiasCross Then

'BiasAmount = CInt (InputBox("Bias amount? (1 to 5)"))

'End If

b.Clear(Color.White)

' $\mathrm{x}$ and $\mathrm{y}$ are the center

$\mathrm{x}=10+\operatorname{cross} \backslash \backslash 2$

$y=10+\operatorname{cross} 1 \backslash 2$

While y $<=$ imagesize -10 - cross $\backslash 2$

While $\mathrm{x}<=$ imagesize -10 - crossl $\backslash 2$

b.FillRectangle(Brushes.Black, $\mathrm{x}$ - crossl $\backslash 2$, y - crossw $\backslash 2$, crossl, crossw)

b.FillRectangle(Brushes.Black, $\mathrm{x}$ - crossw $\backslash 2$, y - crossl $\backslash 2$, crossw, crossl)

If DistortCross $=$ MsgBoxResult.Yes Then 


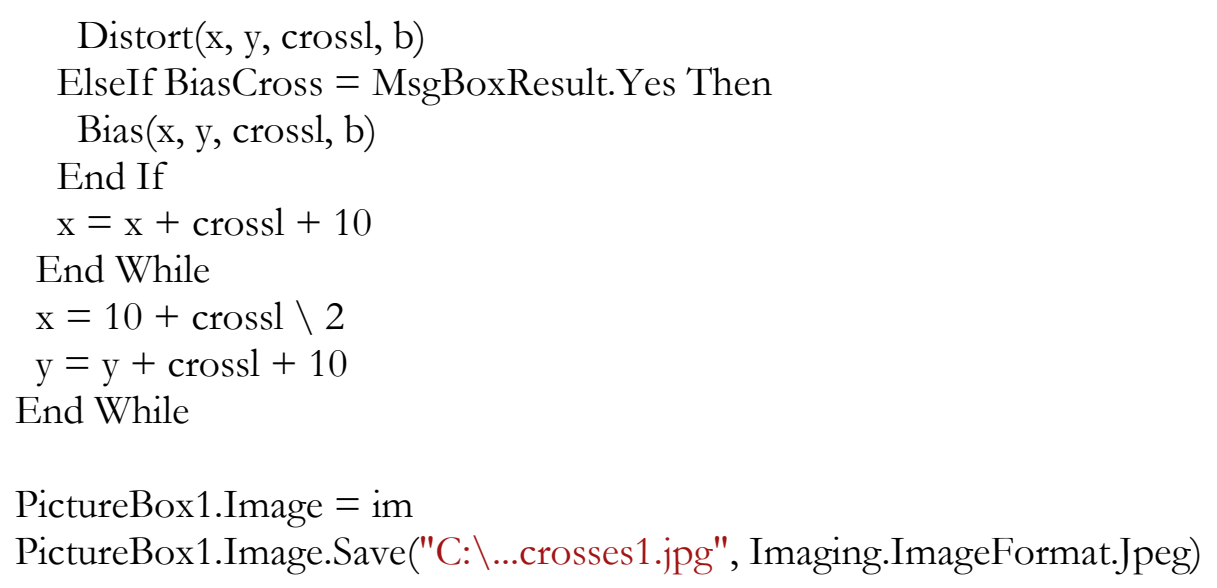

End Sub

Private Sub PictureBox1_MouseEnter(ByVal sender As Object, ByVal e As System.EventArgs) Handles PictureBox1.MouseEnter

PictureBox1.BackColor $=$ Color.Blue

PictureBox1.BorderStyle $=$ BorderStyle.FixedSingle

End Sub

Private Sub Distort(ByVal x As Integer, ByVal y As Integer, ByVal 1 As Integer, ByRef $\mathrm{f}$ As Graphics)

' $\mathrm{x}$ and $\mathrm{y}$ locate the center of the cross, each arm of which is 2 units wide, 10 units long.

' Pick a random 1x1 square to turn white

' Randomly choose to distort horiz or vert arm of cross.

Dim blankx, blanky As Integer

If $\operatorname{Rnd} 0<=0.5$ Then

' choose horizontal, $x$ range from $x-5$ to $x+4$, y range from $y-1$ to $y$

blankx $=\operatorname{CInt}(\operatorname{Int}((10 * \operatorname{Rnd} 0)-5))$

blanky $=\operatorname{CInt}(\operatorname{Int}((2 * \operatorname{Rnd}())-1))$

Else

' choose vertical, $\mathrm{x}$ range from $\mathrm{x}-1$ to $\mathrm{x}, \mathrm{y}$ range from $\mathrm{y}-5$ to $\mathrm{y}+4$

blankx $=\operatorname{CInt}(\operatorname{Int}((2 * \operatorname{Rnd}())-1))$

blanky $=\operatorname{CInt}(\operatorname{Int}((10 * \operatorname{Rnd} 0)-5))$

End If

' Now blankx and blanky are number of units to offset from center. One unit = length/10

blankx $=$ blankx $*(1 \backslash 10)$ 
blanky $=$ blanky $*(1 \backslash 10)$

f.FillRectangle(Brushes. White, blankx $+\mathrm{x}$, blanky $+\mathrm{y}, 1 \backslash 10,1 \backslash 10)$

' Now pick a random square adjacent to the cross to turn black

Dim blackx, blacky As Integer

If Rnd $0<=0.5$ Then

'choose horizontal, $\mathrm{x}=-5,-4,-3,-2,1,2,3,4 ; \mathrm{y}=-2$ or 1

Do

blackx $=\operatorname{CInt}(\operatorname{Int}((10 * \operatorname{Rnd}))-5))$

Loop Until blackx $<-1$ Or blackx $>0$

blacky $=\operatorname{CInt}(3 * \operatorname{Int}(2 * \operatorname{Rnd}())-2)$

Else

'choose vertical, $\mathrm{y}=-5,-4,-3,-2,1,2,3,4 ; \mathrm{x}=-2$ or 1

Do

blacky $=\operatorname{CInt}(\operatorname{Int}((10 * \operatorname{Rnd} 0)-5))$

Loop Until blacky $<-1$ Or blacky $>0$

blackx $=\operatorname{CInt}(3 * \operatorname{Int}(2 * \operatorname{Rnd}())-2)$

End If

blackx $=$ blackx $*(1 \backslash 10)$

blacky $=$ blacky $*(1 \backslash 10)$

f.FillRectangle(Brushes.Black, blackx $+\mathrm{x}$, blacky $+\mathrm{y}, 1 \backslash 10,1 \backslash 10)$

End Sub

Private Sub Bias(ByVal x As Integer, ByVal y As Integer, ByVal 1 As Integer, ByRef f As Graphics)

' $x$ and $y$ locate the center of the cross, each arm of which is 2 units wide, 10 units long.

' Pick a random $1 \times 1$ square to turn white

' Randomly choose to distort horiz or vert arm of cross.

Dim blankx, blanky As Integer

If Rnd $0<=0.5$ Then

' choose horizontal, $\mathrm{x}$ range from $\mathrm{x}-5$ to $\mathrm{x}+4$, y range from $\mathrm{y}-1$ to $\mathrm{y}$

blankx $=\operatorname{CInt}(\operatorname{Int}((10 * \operatorname{Rnd}(0)-5))$

blanky $=\operatorname{CInt}(\operatorname{Int}((2 * \operatorname{Rnd}())-1))$

Else

' choose vertical, $\mathrm{x}$ range from $\mathrm{x}-1$ to $\mathrm{x}, \mathrm{y}$ range from $\mathrm{y}-5$ to $\mathrm{y}+4$

blankx $=\operatorname{CInt}(\operatorname{Int}((2 * \operatorname{Rnd}())-1))$ 


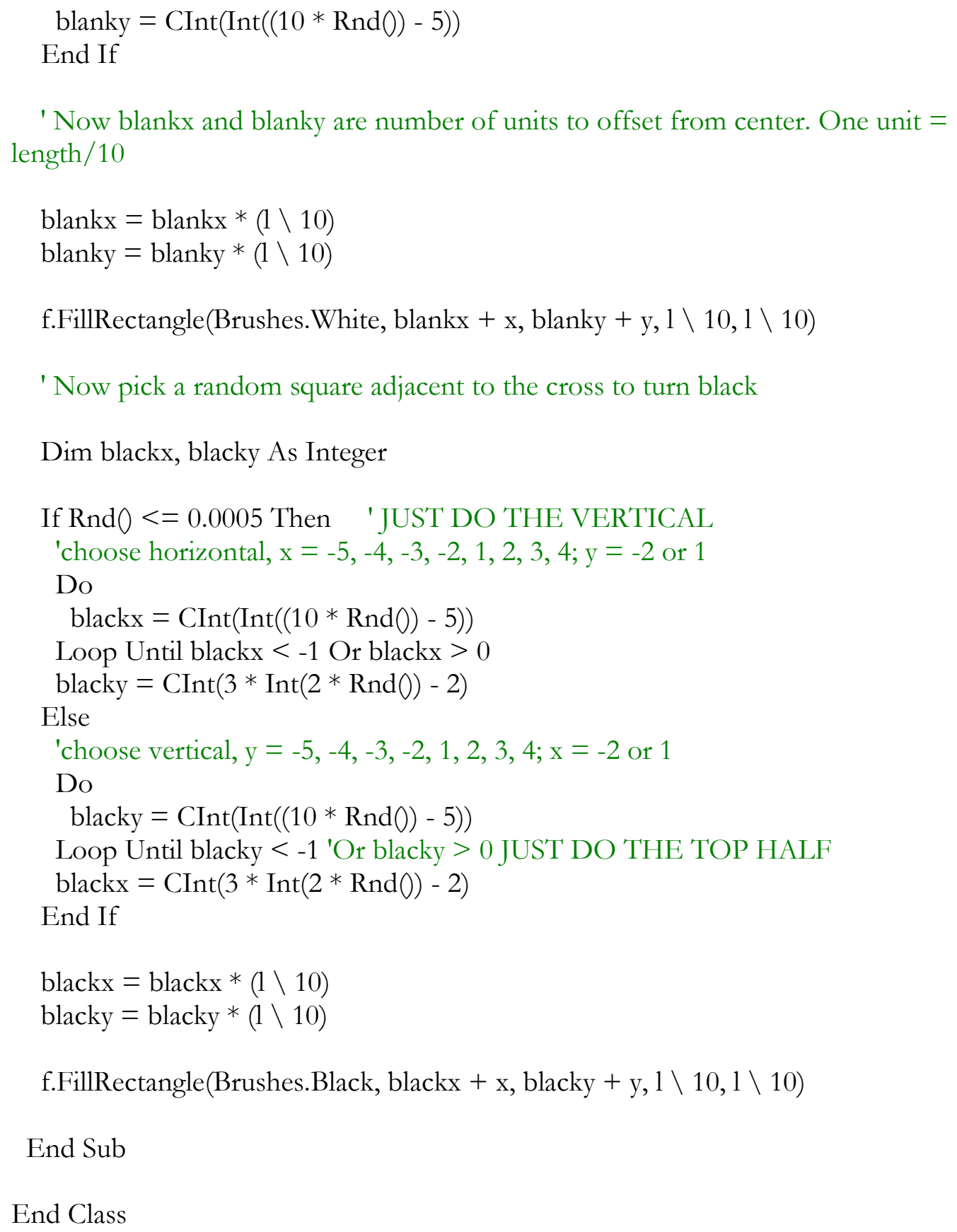

' Now blankx and blanky are number of units to offset from center. One unit = length/10

blankx $=$ blankx $*(1 \backslash 10)$

blanky $=$ blanky $*(1 \backslash 10)$

f.FillRectangle(Brushes. White, blankx $+\mathrm{x}$, blanky $+\mathrm{y}, 1 \backslash 10,1 \backslash 10)$

' Now pick a random square adjacent to the cross to turn black

Dim blackx, blacky As Integer

If Rnd $0<=0.0005$ Then 'JUST DO THE VERTICAL

'choose horizontal, $x=-5,-4,-3,-2,1,2,3,4 ; y=-2$ or 1

Do

blackx $=\operatorname{CInt}(\operatorname{Int}((10 * \operatorname{Rnd}())-5))$

Loop Until blackx $<-1$ Or blackx $>0$

blacky $=\operatorname{CInt}(3 * \operatorname{Int}(2 * \operatorname{Rnd}())-2)$

Else

'choose vertical, $y=-5,-4,-3,-2,1,2,3,4 ; x=-2$ or 1

Do

blacky $=\operatorname{CInt}(\operatorname{Int}((10 * \operatorname{Rnd}))-5))$

Loop Until blacky $<-1$ 'Or blacky $>0$ JUST DO THE TOP HALF

blackx $=\operatorname{CInt}(3 * \operatorname{Int}(2 * \operatorname{Rnd}())-2)$

End If

blackx $=$ blackx $*(1 \backslash 10)$

blacky $=$ blacky $*(1 \backslash 10)$

f.FillRectangle(Brushes.Black, blackx $+\mathrm{x}$, blacky $+\mathrm{y}, 1 \backslash 10,1 \backslash 10)$

End Sub

End Class 


\section{Appendix B -- Complex number notation}

Complex numbers can be expressed in two equivalent formats.

Let $z=x+y i$, a complex number.

Then the real portion of $z, \operatorname{Re}(z)=x$ and the imaginary portion, $\operatorname{Im}(z)=y$. The number $z$ has a magnitude $|z|=\sqrt{x^{2}+y^{2}}$ which is the "length" of the number.

If one sets $\mathrm{r}=|z|$ and $\theta=\operatorname{arctangent}(y / x)$ where a branch of the arctangent function is denoted, for example $(-\pi, \pi]$ or $[0,2 \pi)$, then $r e^{i \theta}$ is a unique way of writing the number $z$.

To convert back to $x+y i$ notation, let $\mathrm{x}=r \cos (\theta)$ and $\mathrm{y}=r \sin (\theta)$. This is consistent with the Euler formula,

$e^{i \theta}=\cos (\theta)+i \sin (\theta)$

The benefit of the $r e^{i \theta}$ is apparent when complex numbers need to be multiplied and divided. Performing these operations on numbers notated $x+y i$ is cumbersome and prone to human error due to the number of operations, while doing so on numbers notated $r e^{i \theta}$ requires merely adding or subtracting of $\theta$ and multiplying or dividing $r$. 